\title{
Predicting Interference Patterns between Offshore Wind Farms through Wake Analysis
}

\author{
by

\section{Melissa Breanna Matis, B.S.} \\ A thesis submitted to the Faculty of Graduate and Postdoctoral Affairs \\ in partial fulfillment of the requirements for the degree of
}

Master of Applied Science in Sustainable Energy Engineering

Department of Mechanical and Aerospace engineering

Carleton University

Ottawa, Ontario

April 2017

(C) Copyright

Melissa Breanna Matis, 2017 


\begin{abstract}
This research is to characterize the wake effect from a cluster of offshore wind turbines with the purpose of optimizing spacing between separate farms to avoid wake interference. Placing wind turbines within a field of disturbed flow decreases power output, and in some cases, threatens structural integrity. Unsteady loading and fluxes in wind turbine power generation are born out of turbulence and can further lead to a reduction in the overall energy production of a wind farm. As the offshore wind industry exhibits rapid growth, an immediate need for standards that enable optimization of farm placement for offshore development exists. Wake characterization was accomplished in this research through computational fluid dynamics (CFD) modelling. ANSYS CFX was used in this research to simulate the wake produced by offshore wind turbine arrays located near the shores of Main Duck Island in Lake Ontario using full rotor geometry representation. These offshore wind farms are currently in the planning, preconstruction phase, and there is no raw data for validation of the CFD model so wind tunnel testing was done to validate the modelling techniques employed in the array simulations. A percent error of $9.5 \%$ between the two data sets was achieved. Three different array configurations were simulated in ANSYS, each containing a different number of rows. The wake $7 \times 4$ array had a recovery distance of approximately 130 rotor diameters. Approximately $89 \%$ recovery is achieved at $6.8 \mathrm{~km}$ downstream from the $6 \times 4$ array and at $9 \mathrm{~km}$ downstream from the $7 \times 4$ array. A comparison simulation was run using the $7 \times 4$ array with an increased inlet speed. Results of this simulation revealed a $23 \%$ increase in wake recovery length. Strategically informing offshore wind farm siting from wake research will maximize power extraction from clean available resources.
\end{abstract}




\section{Acknowledgments}

I would like to thank my thesis supervisors Fred Afagh and Craig Merrett for their guidance, support, and patience. Thank you for giving me the opportunity to pursue this research. I have learned a great deal from both of you during my time at Carleton. I am also very grateful for the financial support provided to this work by NSERC and Trillium Wind Power.

Many thanks to colleagues Kenny Lee Slew for his CFD advice, Vien Nguyen for her assistance in the wind lab and graciously sharing equipment with me, and to Doma Slaman for his guidance on hot wire wake analysis. Thank you to Neil McFadyen for his support with computer resources for CFD simulations and data collection in the wind lab. I am also grateful to Ian Lloy and Alex Proctor who were always willing to lend tools and helped manufacture the base structure needed for the wind tunnel experimentation.

I am also thankful for the outlet I had during my school work through rowing. Thank you to my supportive coaches and teammates of the Carleton Ravens Rowing team. Early mornings on the Ottawa River with the crew were a much needed outlet from the rigors of grad studies.

I am so thankful for my partner Brad Barnes, not only for his steadfast support and humour, but also for his help with using Linux software to run simulations over command line, assistance in moving lab equipment, and the insightful comments provided on the technical aspects of this thesis during the editing process. Thank you to my family and friends for their love and support. My selfless parents Dennis and Sylvia, helped edit this thesis and my sister Sierra, was gracious enough to share her policy expertise. I thank God for the unwavering strength he provided me to keep pursing this work to the best of my abilities despite moments of exhaustion. I could not have finished this work alone. 


\section{Table of Contents}

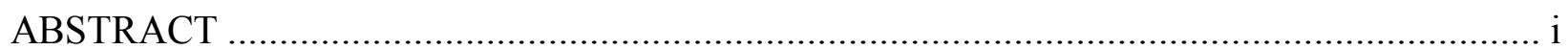

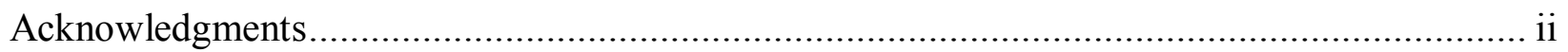

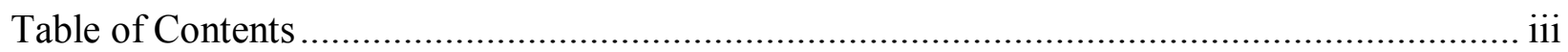

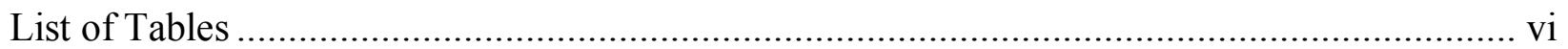

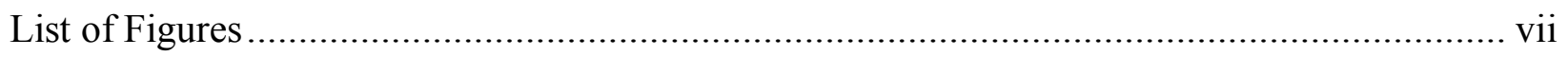

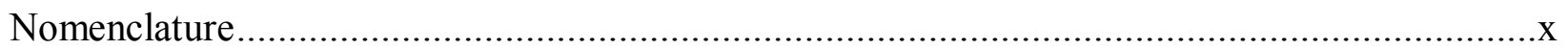

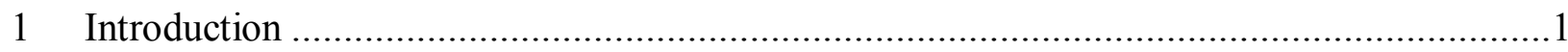

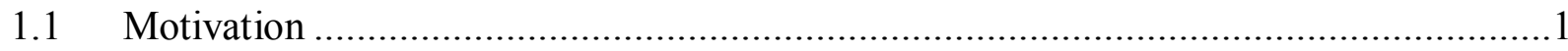

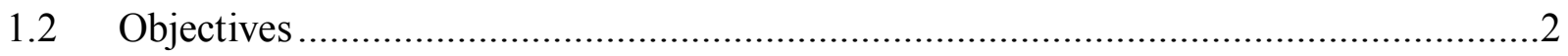

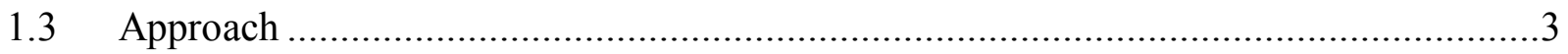

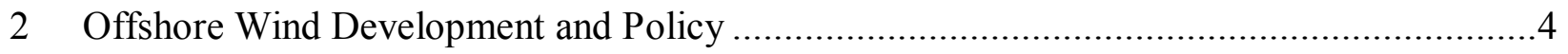

2.1 Development in Europe and the United States ..........................................................

2.2 Issues Regarding Wind Power Development in Canada................................................5

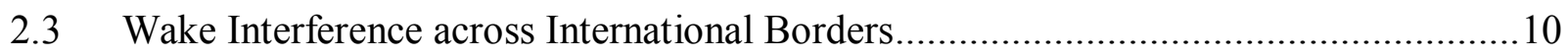

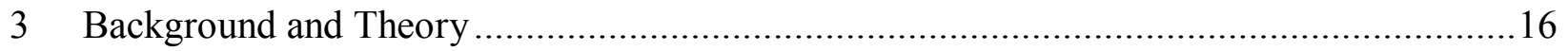

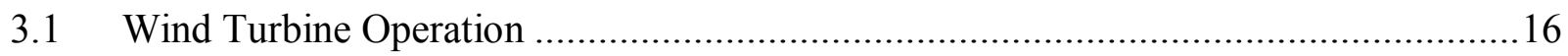

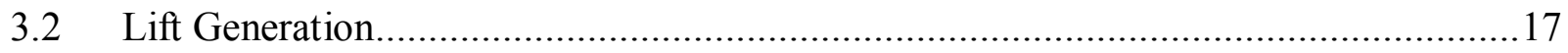

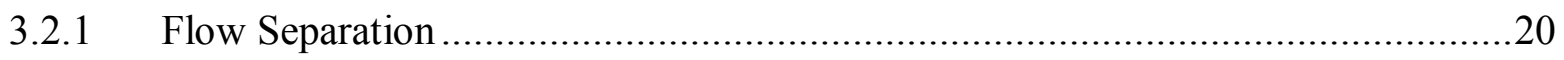

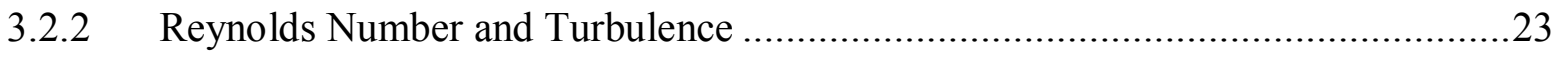


3.2.3 Physical Structures in Turbulent flow

$3.3 \quad$ Wake Simulation through CFD ..................................................................... 31

3.3.1 Simplifications to Fluid Equations ....................................................... 32

3.3.2 Approximating the Navier-Stokes Equations ...............................................34

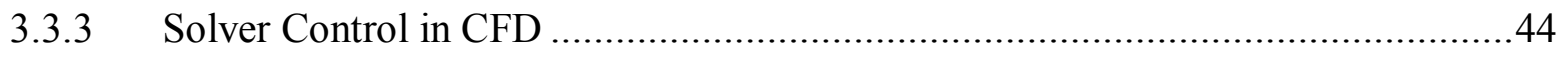

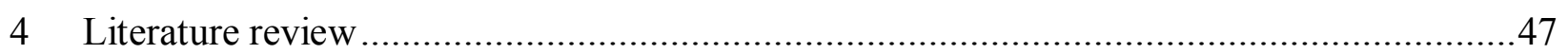

4.1 Previous Work in Offshore Wind Farm Wake Analysis ......................................47

4.2 Rotor Geometry Modelling Techniques in CFD .............................................49

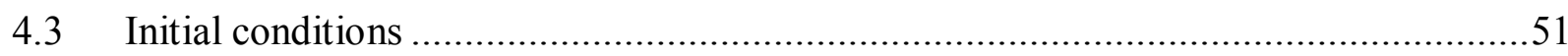

4.4 Previous Work in Experimental Wake Characterization ..........................................52

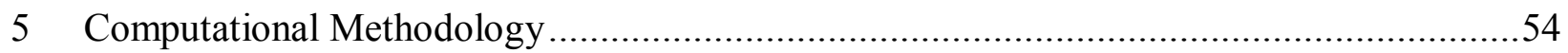

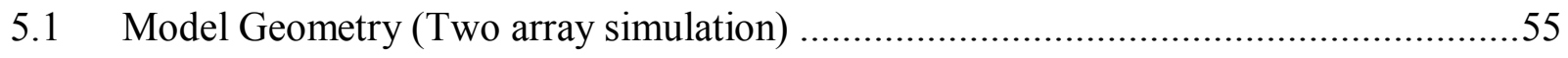

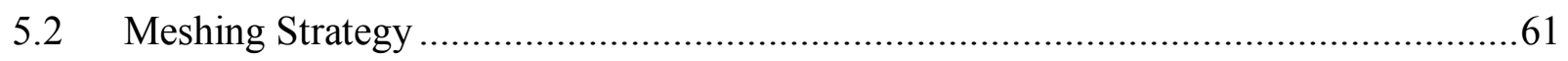

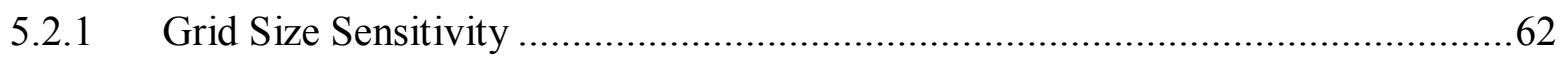

5.2.2 Mesh Adaptation for Vortex Shedding .....................................................64

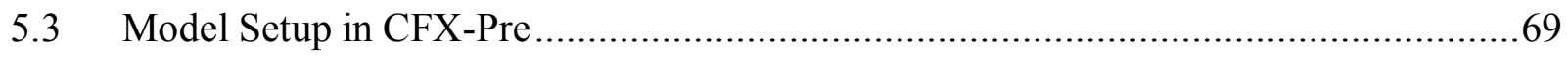

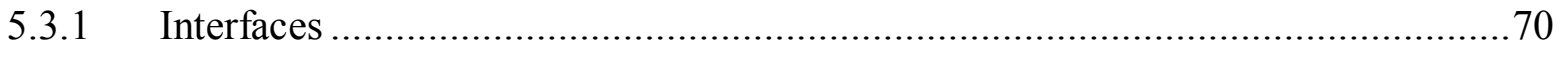

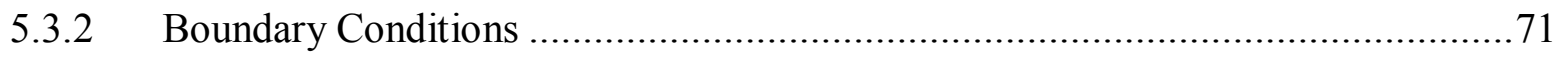

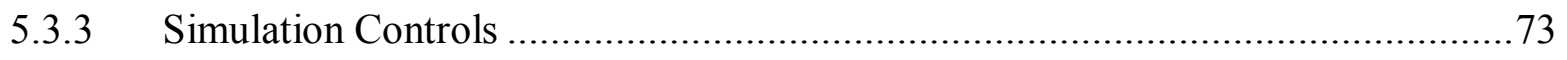

$5.3 .4 \quad$ Time ${ }^{\prime}$ 
5.4 Rotor Size Comparison Simulation ............................................................. 81

5.5 Additional Simulations........................................................................... 82

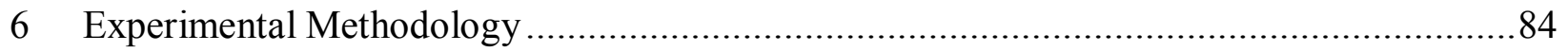

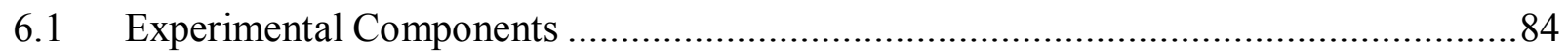

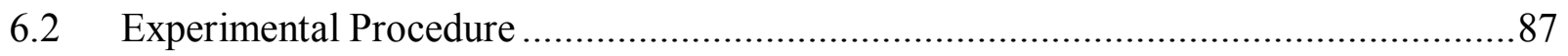

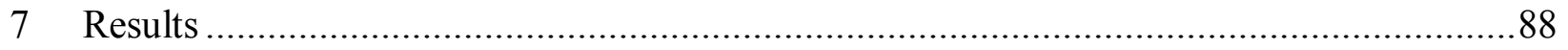

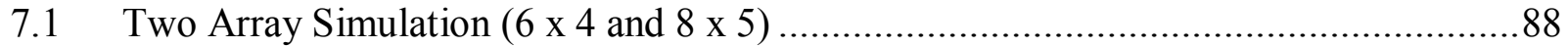

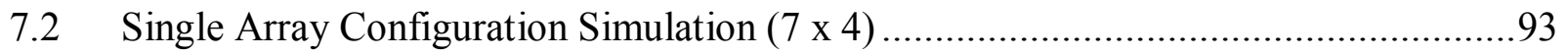

7.3 Increased Inlet Speed for Single Array Simulation $(7$ x 4) ................................96

7.4 Influence of Rotor Geometry on Wake Recovery Distance ....................................99

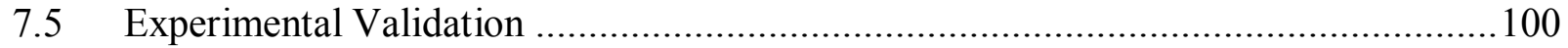

7.6 Convergence Levels ........................................................................... 103

8 Conclusion and Future Work Recommendations ................................................ 105

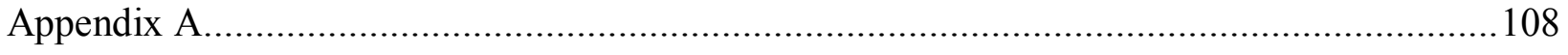

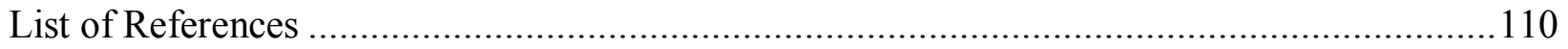




\section{List of Tables}

Table 3.1 Classification of Prominent Turbulence Models ...................................................39

Table 3.2 Turbulence Model Comparison Chart .........................................................43

Table 5.1 Wind Turbine Specifications Comparison [96], [123] .......................................57

Table 5.2 Sandia NASA-Langley Blade Specifications [96] ................................................58

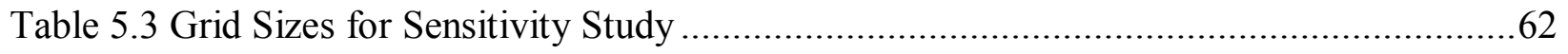

Table 7.1 Percent Differences; Experimental vs. CFD Simulation Data ................................ 101

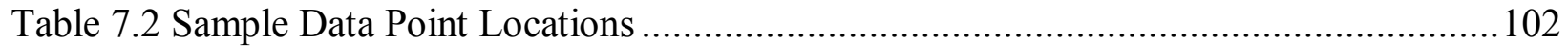

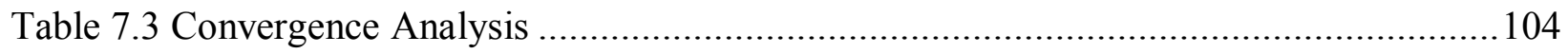




\section{List of Figures}

Figure 2.1 Potential U.S. and Canada Offshore Wind Farm Sites in the Lake Ontario [125] ......10

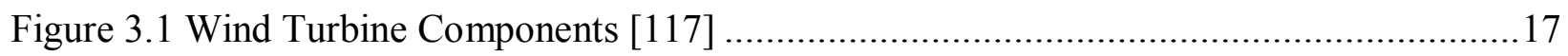

Figure 3.2 Pressure Distribution Normal to a Curved Surface ...........................................19

Figure 3.3 Lift on an Airfoil Due to Pressure Distribution ....................................................20

Figure 3.4 Regions of the Boundary Layer [42] .................................................... 21

Figure 3.5 Boundary Layer Separation on an Airfoil [44] ..........................................22

Figure 3.6 Flow Visualization of Vortex Shedding [46] ...................................................23

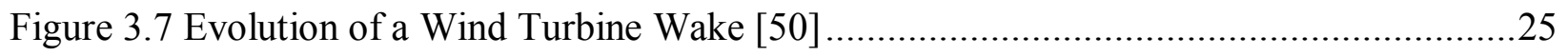

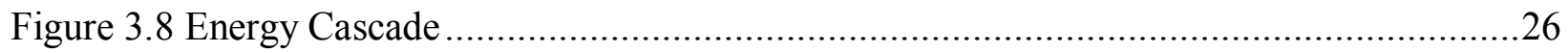

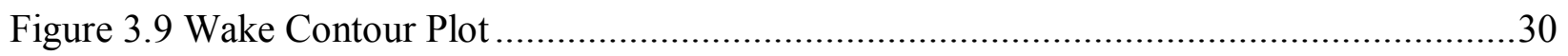

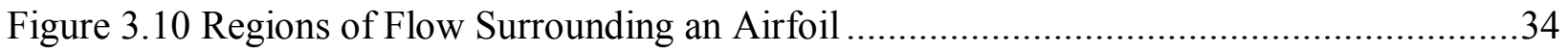

Figure 3.11 Simulation Capacity of the Main Turbulence Models [51] ...............................36

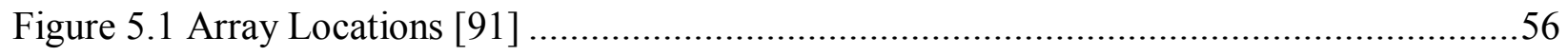

Figure 5.2 Full Rotor Geometry Creo Build .................................................................59

Figure 5.3 Two-Array Simulation Geometry ...........................................................60

Figure 5.4 Rotational Domains inside the Stationary Environment .................................61

Figure 5.5 Grid Sensitivity Study Velocity Plots .....................................................63

Figure 5.6 Percent Differences of the Grid Sensitivity Study ..........................................64

Figure 5.7 Mesh Configuration of a Rotational Domain in the Simulation .............................65

Figure 5.8 Hybridized Mesh Surrounding a Blade Element ...........................................66

Figure 5.9 Two-Array Simulation Domain Mesh..........................................................69

Figure 5.10 Boundary Conditions for the Two Array Simulation .....................................73 
Figure 5.11 Velocity Comparison Plots from Time Step Study..... .80

Figure 5.12 Percent Difference between Nodes of the Largest and Smallest Time Step .81

Figure 6.1 Experimental Procedure Flowchart .85

Figure 6.2 Model Wind Turbine Used in Experimental Testing .86

Figure 6.3 Hot Wire Probe.... .86

Figure 7.1 Average Wake Velocity across Rotor Position behind Array 1 from Simulation Results .89

Figure 7.2 Average Wake Velocity across Rotor Position behind Array 2 from Simulation Results .90

Figure 7.3 Cumulative Wake Effect inside Array 1 from Simulation Results ..........................91

Figure 7.4 Cumulative Wake Effect Velocity Contour Plot from Simulation Results ................92

Figure 7.5 Turbulent Kinetic Energy in the Wake of a Single Turbine behind the Sixth Row, in the

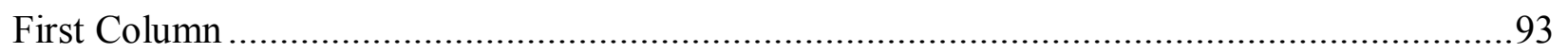

Figure 7.6 Average Wake Velocity across Rotor Position behind 7 x 4 Array from Simulation

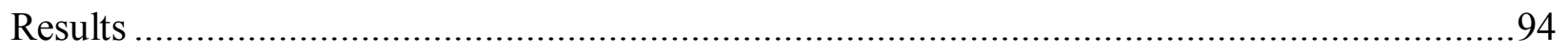

Figure 7.7 Cumulative Wake Effect Velocity Contour Plot for 7 x 4 Array from Simulation Results

Figure 7.8 Typical Turbulent Kinetic Energy in the Wake of a Single Turbine behind the Seventh

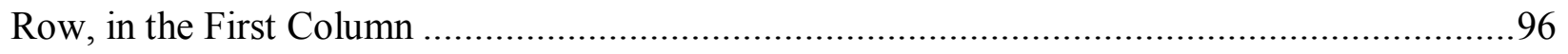

Figure 7.9 Average Wake Velocity across Rotor Position behind 7 x 4 Array with Increased Inlet Wind Speed from Simulation Results .97

Figure 7.10 Cumulative Wake Effect Velocity Contour Plot for 7 x 4 Array with Increased Inlet

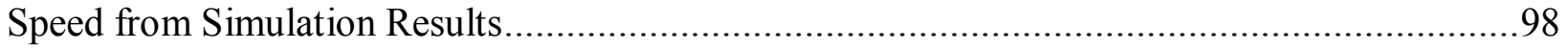


Figure 7.11 Average Wake Velocity across Rotor Position behind Large Rotor (154 m) from Simulation Results

Figure 7.12 Average Wake Velocity across Rotor Position behind Small Rotor (105 m) from

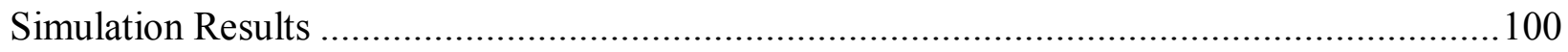

Figure 7.13 Experimental Data Ranges Compared with CFD Data. (Image source: [121]).......102 


\section{Nomenclature}

\section{Latin Letters}

\begin{tabular}{|c|c|c|}
\hline$a_{n}$ & Particle acceleration in the normal direction & $\underline{m^{2}}$ \\
\hline$a_{s}$ & $\begin{array}{l}\text { Particle acceleration in the stream wise } \\
\text { direction }\end{array}$ & $\frac{m^{2}}{s}$ \\
\hline c & Chord length & $m$ \\
\hline$e_{i j}$ & Dissipation tensor for the smallest eddy & $\frac{1}{S}$ \\
\hline$h$ & Height & $m$ \\
\hline$h_{r e f}$ & Reference Height & $m$ \\
\hline$i, j$ & Components in the $i$ and $j$ direction & - \\
\hline$k$ & Kinetic energy extraction rate & $\frac{m^{2}}{s^{2}}$ \\
\hline$l_{m i x}$ & Mixing length & $m$ \\
\hline$n$ & Distance normal to a curved surface & $m$ \\
\hline$p$ & Pressure & $P a$ \\
\hline$s$ & Distance & $m$ \\
\hline$t$ & Time & sec \\
\hline$t_{L}$ & Timescale of the largest eddies & sec \\
\hline$t_{\eta}$ & Timescale of the smallest eddies & sec \\
\hline$U$ & Velocity & $\frac{m}{s}$ \\
\hline$u_{*}$ & Friction velocity at the nearest wall & $\underline{m}$ \\
\hline
\end{tabular}




$\begin{array}{clc}u, v, w & \text { Velocities components for } \mathrm{x}, \mathrm{y}, \mathrm{z} \text { directions } & \frac{m}{\mathrm{~s}} \\ V_{\text {Int. }} & \text { Velocity of largest eddies } & \frac{m}{\mathrm{~s}} \\ V_{\text {Kol. }} & \text { Velocity of smallest eddies } & \frac{m}{\mathrm{~s}} \\ \mathrm{Y} & \text { Distance to the nearest wall } & m \\ \mathrm{Y}^{+} & \text {Y Plus, used in near wall node placement } & -\end{array}$




\section{Greek Letters}

$\alpha$

$\varepsilon$

$\eta$

$\theta$

$\kappa$

$\mu$

$\mu_{T}$

V

$\pi$

$\rho$
Wake expansion coefficient

Rate of dissipation of turbulent kinetic $\frac{m^{2}}{s^{3}}$

energy

Length scale of smallest eddies

$m$

Angle

degrees

Streamline curvature

$\frac{d \theta}{d s}$

Viscosity

$\frac{k g}{m * s}$

Eddy-viscosity

Kinematic viscosity

$\frac{m^{2}}{s}$

$\frac{m^{2}}{s}$

Constant variable, pi

Density $\frac{\mathrm{kg}}{\mathrm{m}^{3}}$ 


\begin{tabular}{|c|c|}
\hline \multicolumn{2}{|c|}{ Abbreviations } \\
\hline$A B L$ & Atmospheric Boundary Layer \\
\hline AESO & Alberta Electric System Operator \\
\hline$B L$ & Boundary Layer \\
\hline$C F D$ & Computational Fluid Dynamics \\
\hline$C F L$ & The Courant-Friedrichs-Lewy condition \\
\hline CTA & Constant temperature anemometer \\
\hline$D A Q$ & Data acquisition system \\
\hline$D E S$ & Detached Eddy Simulation \\
\hline$D N S$ & Direct Numerical Simulation \\
\hline$E U$ & European Union \\
\hline$E W E A$ & European Wind Energy Association \\
\hline$G E G E A$ & Green Energy and Green Economy Act \\
\hline$G G I$ & General Grid Interface \\
\hline$H A W T$ & Horizontal Axis Wind Turbine \\
\hline IESO & Independent Electricity System Operator \\
\hline IJC & International Joint Commission \\
\hline$L E S$ & Large Eddy Simulation \\
\hline$M W$ & Megawatt \\
\hline$N A S A$ & National Aeronautics and Space Administration \\
\hline NREL & National Renewable Energy Lab \\
\hline
\end{tabular}




$\begin{array}{ll}\text { RANS } & \text { Reynolds-Averaged Navier-Stokes } \\ R E M & \text { Rapid Eye Movement } \\ R P M & \text { Revolutions per minute } \\ R S M & \text { Reynolds stress transport model } \\ S A R & \text { Synthetic aperture radar } \\ S G S & \text { Sub-grid-scale model } \\ \text { SST } & \text { Shear stress transport } \\ \text { TSR } & \text { Tip speed ratio }\end{array}$




\section{Introduction}

The research is to characterize the wake effect from a cluster of large-scale offshore wind turbines in order to optimize spacing between separate farms. Optimized spacing prevents significant power losses incurred from wake-farm interactions. Computational Fluid Dynamics (CFD) simulations performed in ANSYS CFX were used to characterize the wake effects of two proposed offshore sites near Lake Ontario's Main Duck Island. This research also included a policy component which examined some of the key factors that have likely affected the halt of offshore wind power projects in Ontario. The motivation of the technical component of this research is discussed in more detail in Section 1.1, followed by the objectives of the research in Section 1.2. Policy aspects of offshore wind development are discussed in Chapter 2. The conclusion of this chapter outlines the approach taken towards the CFD and experimental wake characterization carried out in the research. The rest of the thesis includes a Background and Theory section in Chapter 3, followed by a Literature Review of previous work in Chapter 4 . Methodology is discussed in Chapters 6 and 5, followed by Results in Chapter 7. The thesis conclusion and future work recommendations are presented in Chapter 8 .

\subsection{Motivation}

The motivation of this research is to improve offshore wind farm siting by avoiding the risks to power production imposed by wake effects. An increased number of offshore sites creates the potential for unwanted interference between farms due to the cumulative wake effects of clusters of wind turbines. In North America, strategic offshore wind farm development has recently become a priority. The U.S. is investing in offshore wind energy research and development as it has been shown in recent studies that this form of energy is a viable source. In 2016, the U.S. Department of Energy reported that the U.S. has a total of 4,000 GW in offshore wind resources 
[1]. This research seeks to analyze the wake effects of proposed offshore wind farms along the U.S- Canada border in Lake Ontario. Wind resources and bathometry in several regions of the Great Lakes provide ideal locations for offshore farms; however, developers are uncertain if these potential sites lie an adequate distance away from each other in order to avoid interference.

Power losses arise if a single wind turbine's wake impinges on the operation of another turbine. The extent to which energy production is impeded from wind turbine interactions with wakes varies as there are multiple factors of influence such as the locations and layouts of wind farms as well as atmospheric stability [2]. An early study done in 1985, for three turbines placed seven rotor diameters downstream of each other, documented the averaged power losses in a downstream row to be $10 \%$ [3]. A more recent study, found that the energy losses in a downstream turbine can fall into much higher ranges, nearing $40 \%$ due to wake effects and atmospheric stability [4].

High construction costs are often a major roadblock to growth in the offshore wind energy industry. These costs are significantly higher than for land based wind energy as towers and foundations can cost roughly 2.5 times the price of those for an onshore project of a similar size [5]. However, offshore wind offers significant advantages over its land counterpart. On top of the potential of a larger power production capacity due to the availability of steadier, stronger winds, many offshore wind farms can be placed close to large cities centres, reducing the need for extremely long transmission line construction from rural locations. These benefits unique to offshore wind, encourage research into optimizing siting to improve the industry.

\subsection{Objectives}

The objective of this research is to gain understanding about optimal spacing between offshore wind farms to inform wind farm development in Lake Ontario for an Ontarian energy company; 
Trillium Wind Power. To achieve this objective, characterization of the overall wake effect of entire offshore wind farms is necessary. Wake studies were conducted using full rotor CFD simulations and experimental testing was done for validation of the techniques implemented to produce the CFD simulation. In addition to the technical fluid dynamics research, policy issues surrounding wind farm development in Canada were also researched and are presented in Chapter 2.

\subsection{Approach}

The scientific research consists of two components: Full rotor CFD simulations of offshore windfarm clusters and experimental validation of the CFD simulation through wind tunnel testing. Wind conditions specific to the proposed Lake Ontario build sites were used to simulate the wind farms in CFD. Average wind speed data from Lake Ontario was gleaned from the 2007 Ontario Wind Atlas published by the Ministry of Natural Resources. Wind data for 20 years before the 2007 study shows that average wind speeds over this time period have remained consistent. The experimental test case, which will be described in detail in Chapter 6, was replicated in an ANSYS CFX simulation to produce a validation study of CFD full rotor methodology. The results of the experimental wind tunnel test and the corresponding CFD simulation were in close agreement. The same simulation settings, meshing approach, rotor representation, convergence criterion and turbulence model used in the CFD simulation for the wind tunnel experiment was implemented in the full scale offshore CFD simulations. In addition to the scientific research component, the policy factors influencing offshore wind development were researched and are presented in Chapter 2. 


\section{Offshore Wind Development and Policy}

Policy influence on offshore development was researched through a literature review presented in this Chapter. Europe's advanced wind energy industry and the recent American offshore project is discussed in Section 2.1. Canada has not experienced the influx of wind power that Europe has, where political factors have impeded development of offshore wind. These factors are discussed in Section 2.2. The way in which the scientific work of this research could positively influence the policy of offshore wind in Canada is also discussed in Section 2.3.

\subsection{Development in Europe and the United States}

Over the past decade, the offshore wind industry has experienced steady growth in Northern Europe, and the number of independent farms is still increasing [6]. As a result, a limited number of nearshore build locations in waters less than $30 \mathrm{~m}$ deep, where foundations can be fixed to the marine floor, exist in Northern Europe. This has pushed development plans towards deeper waters where the wind often blows stronger with more consistency. Far offshore wind farms are constructed in waters with depth ranges of $30 \mathrm{~m}-50 \mathrm{~m}$ deep. Foundations of far shore wind turbines have to be deployed on floating rafts, bringing about a range of complex forces to solve from the vibration of the raft at the tower base through wave motion. The first far offshore turbine prototype was completed in Norway in 2009. The success of this project will be built upon this year through the world's first far offshore commercial-scale pilot project in Scottish waters. Far offshore wind energy projects are still in the prototyping phase whereas the nearshore industry is currently widely adopted with the first operational farm, completed over two decades ago in Danish waters [7]. North America experienced the debut of offshore wind in late 2016 with a nearshore farm off the coast of Rhode Island. With five 6 MW turbines, the Block Island wind farm has added $30 \mathrm{MW}$ of clean energy capacity to the grid [8]. 


\subsection{Issues Regarding Wind Power Development in Canada}

Currently, no offshore wind farms exist in Canada despite the excellent offshore resources. All 10,000 MW of wind energy capacity in Canada is on land, with every Canadian province having access to this energy resource [9]. Trillium Wind Power Corp, a local energy company, has studied the offshore wind resources and bathometry of Lake Ontario. A region of the lake near Main Duck Island, close to Kingston Ontario, was found to have ideal wind resources. Additionally, the bathometry of the lake in this region is very conducive to pile driven tower construction due to shallow waters. This construction technique is significantly cheaper than floating structures. If the Trillium project is constructed, the project could add an additional 300 MW or more clean energy capacity to the grid.

Trillium Wind Power proceeded with the official planning process to construct an offshore wind farm in this region of Lake Ontario. The government of Ontario halted the progression of this project in February 2011 by imposing a moratorium on offshore wind projects. The moratorium was fueled by rural residents of the region who were opposed to the construction of a wind farm on the lake [10]. Opposition to wind turbine projects by local members of the community is often driven by a desire to maintain the landscape for property value [11], as well as concerns over health risks of those living near wind farms [12]. The risks that wind farms impose to wildlife such as bats and birds are an additional barrier to development.

There appears to be limited arguments in defence of the aforementioned negative claims regarding wind farms. Some accusations of the health risks of wind farms are that they can cause nausea, vertigo, dizziness, cognitive deficits and cardiovascular disease. Even mental health concerns over wind turbines have arisen which claim that the technology can cause worry, anger and community division. A research study in 2014 qualitatively analyzed negative feelings that rural residents have towards wind turbines in Ontario. Two wind turbine communities were 
studied: Port Burwell and Clear Creek. In these Ontario studies, surveys and interviews were conducted on willing participants living within $1 \mathrm{~km}-2 \mathrm{~km}$ from a wind turbine. Support of wind turbines was much higher in Port Burwell (80\%) than in Clear Creek (63\%). Questions were asked in the surveys and interviews on the impacts of turbines on quality of life. A common response in Port Burwell was related to the noise. Interviewees claimed that most citizens get used to the noise and eventually do not hear it. Clear Creek responses were much more negative, with some citizens indicating that they have difficulties sleeping and enjoy spending time in the outdoors less because of the wind turbines [13].

Societal conflict was another issue that arose amongst citizens of the rural communities. The conflict stemmed from supporters of wind turbines making light of the problems that the opposition claimed to be facing. This disregard for the concerns of the opposition caused the opposed side to feel marginalized. Of the problems claimed by the opposition, health effects were central. Conflict was catalyzed further through the siting and planning stage of the wind turbine projects. Trust was another factor that the survey questions were aimed towards. Supporters of wind turbine development tended to be much more trusting of the local government, wind developers, neighbors and colleagues than those who were opposed. The political climate worked to repress feelings of concern as the siting and design phase happened before the Green Energy and Green Economy Act (GEGEA) legislation which removed municipal veto powers over renewable energy. Taking away municipal veto powers, combined with no stakeholder engagement sessions in the planning stages gave rise to distrust towards developers and feelings of having no choice. The overall conclusions of the study revealed that the absence of a venue for face to face consultation on the siting of wind farms before development, strengthens negative views towards wind farms [13]. 
Aside from potential health risks and negative psychosocial effects, concerns also exist over wind farm effects on avian life. In western Canada, wind farms are eliminating prime nesting areas for birds of prey. Research is currently being carried out by the University of British Columbia, tracking birds of prey migratory patterns and coupling this data with wind patterns [14]. The intent is that this information will inform siting of wind farms and results of the study could influence policy decisions on wind farm development in regions where migratory birds nest. Bats are also threatened by wind turbine development, with documented bat fatalities at a significant number of Canadian and American wind farms. White nose syndrome has already threatened the bat population, so the added danger of wind turbines has made protection a high priority for conservationists [15].

Protecting this species is of interest to Canadians as bats play an integral role in the environment, consuming harmful agricultural pests and mosquitoes. An Albertan study found that it was not collisions with turbine blades that was causing deaths, but severe lung damage caused by extreme pressure changes near the rotors. Bird lungs on the other hand can handle these pressure changes [16]. In 2005, a study on Transalta's Summerview wind farm revealed 20-30 dead bats per turbine. Transalta took this study to heart by implementing more bat friendly operational strategies. By increasing the cut in speeds of the turbines to be above the wind speed ranges that bats will fly in, the bat mortality rate was nearly cut in half [16]. After this study, the province of Alberta enacted a policy requiring developers to produce a risk assessment that indicated the relative risk of bat fatality for a proposed site before construction. A mitigation framework developed by the provincial government will be enacted based upon the risk assessment. Mitigation strategies are focused on the areas of a site where fatality is high and monitoring ensures that the strategies are effective. Cut in speeds can be adjusted or blades can 
be feathered in low wind speeds. In some cases, this may only need to be done during peak migration periods. If monitoring efforts reveal that the aforementioned steps are unsuccessful, power production may have to be curtailed at night, during the migration peak period [17]. A similar process is carried out between the Ontario government and wind farm developers for bat protection [18]. These strategies which promote the protection of avian life on onshore wind farm sites could also be implemented on offshore wind farm sites to reduce environmental impact.

Health risks due to turbine noise is another major concern amongst residents living near wind farms or potential build site. Health Canada claimed that wind turbine noise does not cause health issues, after a 2015 study involving 1,238 homes near wind farms in Ontario and Prince Edward Island. The only negative health effect found in the study linked to the noise, was annoyance [19] [20]. A Ph.D. thesis conducted in 2016, by a University of Waterloo Health Studies and Gerontology researcher, analyzed the health risks imposed by living near a wind farm due to noise pollution. In one aspect of the study, individuals living within $2 \mathrm{~km}$ of a wind farm in rural Ontario were given standard and validated sleep questionnaires. Another aspect of the study focused on sleep diaries which were completed for a week. Objective studies were also conducted through polysomnography and inside noise measurements for two nights in a row. These studies collected data before and after wind turbine operation. Out of those who participated in the survey, only $10 \%$ of the participants in the study reported to be "annoyed or very annoyed" due to wind turbine noise. Measured data on the mean of rapid eye movement (REM) sleep and sleep efficiency showed no change before and after exposure to wind turbine operation. The author of the study cautiously suggested that there are no significant changes of sleep patterns of the individuals who participated in the study [11]. Studies such as the 
aforementioned thesis and Health Canada report are tools that can be used to build trust between wind farm developers and citizens who live near proposed wind farm build sites.

Improved strategies for construction, scour protection around gravity based foundations, skilled laborers, and an increased number of vessels dedicated to offshore wind farm construction have been identified as innovation needs of the offshore wind industry [21], [22]. If Ontario policy is changed in favour of offshore wind, government funding placed towards creating a workforce that is capable of constructing and maintaining offshore wind turbines would be valuable. In Canada, professional expertise exists in the area of offshore oil and gas with involvement in the industry beginning in 1959 at Sable Island [23] . Mooring and anchoring strategies used in the offshore oil and gas industry could be applied to the offshore wind industry for securing far offshore wind turbine structures; this is a potential area for the transfer of labor skills.

Potential build locations in Lake Ontario for offshore wind farms lie on both sides of the Canada-U.S. border. If the Ontario moratorium on offshore wind is not lifted in the near future, the American potential build site could be developed first and the potential for transboundary wake-farm interaction could be cause for concern. In this section, construction strategies, environmental, mental health and physical health issues associated with wind farms have been identified; indicating that offshore wind development in the Great Lakes is not purely an engineering problem. Development of offshore wind requires the attention of policy makers to ensure the construction of Canadian wind farms is done responsibly, taking the concerns of all stakeholders into consideration. The results of this research which predict the extent to which transboundary wake-farm interference could occur, could be useful in informing policies regarding the construction of offshore wind farms along the U.S-Canada border. 


\subsection{Wake Interference across International Borders}

Offshore wind growth in Europe and North America brings about the issue of wake-farm interaction across international borders. The results of this research predict the wake recovery distance of a seven-row offshore wind farm to be $13 \mathrm{~km}$ under the average wind speed conditions of Lake Ontario and near $16 \mathrm{~km}$ when the wind speeds are $10 \%$ above the average. These results indicate that building an offshore wind farm near or on an international border line can damage the wind resources of a neighboring country. The quantitative nature of this information could influence the creation of international policies that adhere to the interests of nations that share offshore borderlines. To date, only one offshore wind farm exists in North America, which is located off the coast of Rhode Island. On Lake Ontario, there exists potential build locations on the shores of Main Duck Island and the Galloo Islands, which lie approximately $17 \mathrm{~km}$ from one another. Figure 2.1 identifies the relative locations of the Canadian Main Duck Island and the American Galloo Islands.

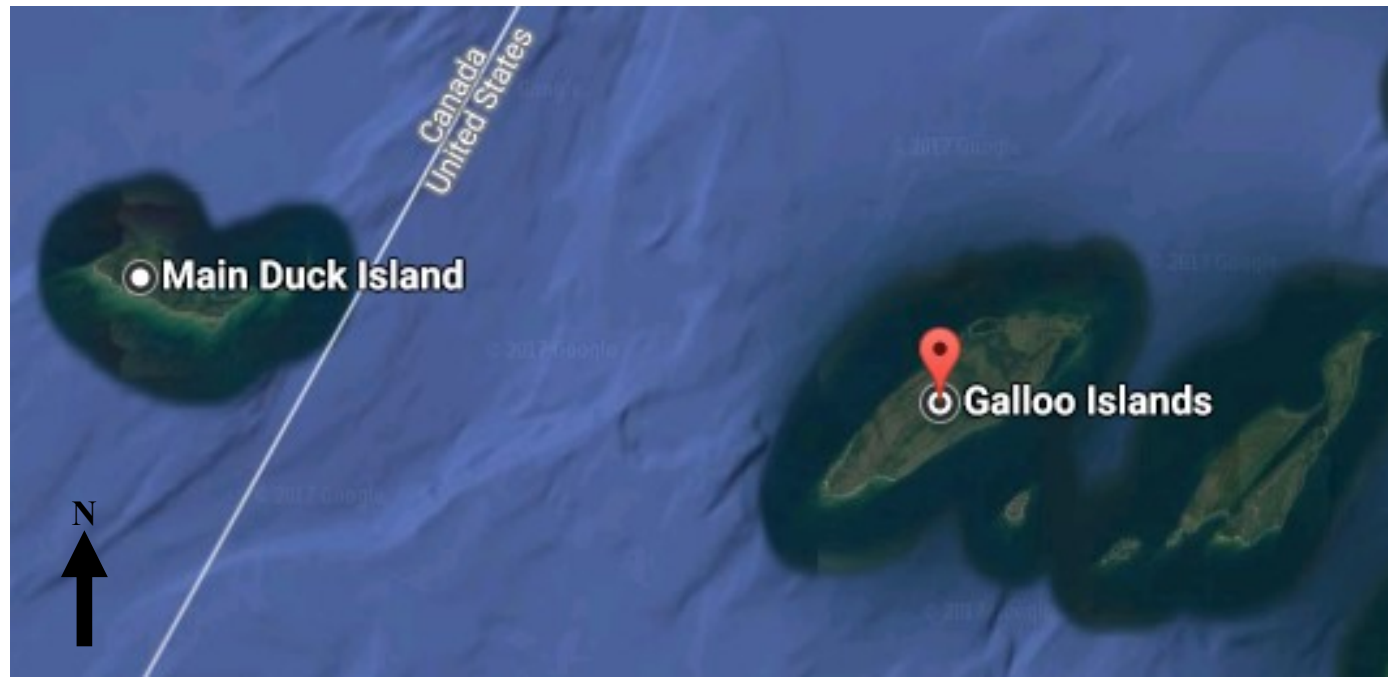

Figure 2.1 Potential U.S. and Canada Offshore Wind Farm Sites in the Lake Ontario [126]

Due to the aforementioned prediction of wake effects stretching over kilometer distances, international policy has to be enacted if the wind resources in this region are to be equally shared 
across the Canada-U.S. border. An example of a long-time established cross-border policy regarding the use of transboundary natural resources is Article VI of the Boundary Waters Treaty of 1909. The Boundary Waters Treaty was signed by the U.S. and Canada to ensure that the water of the St. Mary and Milk Rivers are treated responsibly for the purpose of irrigation and power. This treaty led to the establishment of the International Joint Commission (IJC) as Canadian and American stakeholders recognized that both nations are affected by the other's actions in water systems along the border [24].

The St. Mary River flows through portions of northern Montana and southern Alberta. On both sides of the border, this river is used for irrigation supply and also feeds into a 2 MW capacity hydroelectric generator on the Canadian side [25]. In the province of Alberta, less than half of the moisture required for crop production occurs naturally; therefore, irrigation is required to offset this disparity and to help grow the agricultural industry [26]. The St. Mary River is a crucial irrigation supply vein in Southern Alberta which the Boundary Waters Treaty seeks to protect. From March to May, the United States finds it advantageous to use a greater share of the river's flow than Canada. From June to September, Canada finds it advantageous to use a greater share of the River's supply. Based on the unique irrigation needs of Southern Alberta and Northern Montana, the U.S. is allowed to accumulate an annual $9800 \mathrm{dam}^{3}$ deficit on the St. Mary River during the March to May period. During the June to September period, Canada is allowed to accumulate a $9800 \mathrm{dam}^{3}$ deficit [27]. As in the case of the Boundary Waters Treaty, where Canada and the U.S. draw more than their share of the resources during alternating periods of the year, the U.S. and Canadian offshore wind power producers may also work out a resource sharing schedule where power production periods of the U.S. and Canadian farms occur during different periods of time. 
In addition to the differing irrigation needs of Montana and Alberta, infrastructure such as dams and reservoirs limit the capacity of water that can be diverted from the St. Mary River for irrigation and power production. A similar issue could exist in offshore wind power production. American and Canadian offshore wind power producers in the Great Lakes may find that during long periods of strong winds, power produced may have to be curtailed if the maximum grid and storage capacities have been reached. Based upon weather forecasting and the maximum amount of power that can be absorbed by the grids and storage devices on both sides of the border, policy could be formulated to establish a wind farm operation schedule. When a country has produced the maximum amount of energy that can be absorbed by its storage and grid infrastructure, wind farm operation could be ceased and the farm located on the other side of the border could begin producing power, unobstructed from the wake effects of the wind farm across the border. Such practices could be a part of curtailment management strategies. Curtailment management strategies are a standard component of wind farm operational guidelines which define the maximum amount of power that can be placed onto a grid for a given time period as well as which generators should be shut down during wind power ramp up periods (e.g. during a storm). The Interdependent Electricity System Operator (IESO) of Ontario and the Alberta Electric System Operator (AESO) exhibit best practices for wind power ramp up management strategies [28], [29].

Establishment of policy on offshore wind which ensures that wind farms constructed on both sides of the border lie an adequate distance away from one another to avoid wake-farm interference could be another way to avoid disputes over offshore wind. This could create the need for an international agency dedicated to protecting shared wind resources. Alternatively, the IJC may seek to extend its mandate to include the protection of wind resources for both Canada and the US, as they currently deal with water and air quality issues in the Great Lakes region [30], [31]. 
Presently, the IJC deals with shared water systems and air quality across the U.S. and Canada. The IJC alerts the government about emerging issues that may cause bilateral disputes along the border and works to prevent and resolve disputes [24].

In Europe, the potential for wind farm wakes extending across international borders is a more complex policy issue than in North America. Because of the high number of offshore wind farms in Northern European waters, policies regarding wind farm wake effects which extend across international borders is currently a more immediately pressing issue in Europe than in North America. In an EWEA report, offshore wind farm siting issues was listed as a barrier to development [32]. The current offshore wind capacity of offshore wind farms in Europe is 12.6 MW, a number that is expected to grow significantly over the next three years [33]. A 2020 prediction of capacity for offshore wind installment in Europe is $28 \mathrm{GW}$ in the highest case scenario [34]. This projection is anticipated if the effects of the economic crisis fade and stability is regained to pre-2012 levels. If this economic upturn is not achieved, the lowest case scenario prediction of offshore wind farm capacity is $19.5 \mathrm{GW}$ by 2020 [34].

The greater number of coastal countries in Europe also adds to the complexity of establishing guidelines on wind farm placement; therefore, a "one-size fits all" policy framework may not be practical and in the best interest of European nations which use the ocean. Offshore wind development has the power to affect European policy related to several aspects of the sea and its use, especially the impact on common fisheries, shipping and navigation. WindEurope, formally known and the European Wind Energy Association (EWEA), has acknowledged the need for member states to determine how to manage the interaction between offshore wind technology and their national security needs [35]. 
Environmental matters are transboundary issues. Because the issues of climate change and natural resources are not always confined within borders, the European Union (EU) has jurisdiction over environmental policy. The EU seeks to establish common standards for its member states. An example of the setting of common standards is COP 21, where EU countries as well as other non-union nations met to establish greenhouse gas reduction targets. Policies should be informed through a process of consensus building given that establishing transboundary standards is a challenging endeavor considering that the EU is comprised of 28 member states with diverse national interests and needs. Also, at an institutional level, there are multiple decision making bodies in the EU [36], [37]. Because of this, one common standard establishing offshore farm proximities to international borders may not be feasible. Many of the northern coastal European countries are members of the EU and exhibit strong environmental policy, with ten coastal European countries currently benefitting from offshore wind. Currently, Europe has 12.63 MW of installed offshore wind capacity [38]. Also, the non-member state, Norway and soon to be non-member state, the U.K., exhibit exemplary practices in environment policy. Therefore, if the EU seeks to develop a policy framework to address offshore wind farm siting, the interests of non-member states will also need to be considered.

To formulate a decision which addresses the need to create common standards for offshore wind farm siting, the issue can be transferred from the political sphere of the EU to an agency with applicable technical expertise. This can remove political biases from the decision making process and promote evidence-based policy. WindEurope, formerly known as EWEA, is a technical organization on offshore wind power that seeks to provide quality input into the policy and legislative process [39]. This organization works in partnership with its members and other relevant organizations to promote research, development and innovation of offshore wind with 
the vision of attaining the EU's 2020 targets [39]. WindEurope's technical and political expertise as well as its close partnership with EU members makes it an excellent actor to help input into a policy framework that establishes guidelines for offshore wind farm siting. This framework could advise members on reaching agreements amongst themselves. Policy agreements could be in the form of established distances that wind farms must lie away from international border lines or power production schedules influenced by the local weather patterns and grid absorption capabilities.

In summary, the development of offshore wind farms in Lake Ontario by Canadian and American energy companies may bring about the need for standards regarding wind farm placement and operations. To impose these standards, a governing body that can act at arm's length from the issue would be required. This governing body would work to impose standards ensuring that both countries benefit fairly from the wind resources of the region and help to prevent international disputes over offshore wind. The IJC works to prevent disputes regarding the use of transboundary natural resources and may be an agency with the capacity to deal with offshore wind issues. However, being that the IJC is a governmental organization controlled by countries, this body may be too political to deal with this issue. Alternatively, as in the case of WindEurope which looks after wind energy affairs in the EU, a technical board may be a better type of agency to deal with offshore wind resource sharing in Lake Ontario in an unbiased way. Technical boards specialize in scientific issues while also possessing political expertise, making them excellent actors to help formulate guidelines for offshore wind farm siting. 


\section{Background and Theory}

As stated in Chapter 1, wakes can be destructive to wind turbine structures and detrimental to their power production. The study of wake development is necessary to avoid the aforementioned unwanted effects of wakes. In Sections 3.1, the basics of wind turbine operation from a fluid dynamics perspective along with wake formation will be presented followed by the key physical features which are most important to simulating wakes in Section 3.2. The theory employed by CFD solvers to simulate turbulent flow is discussed in Section 3.3.

\subsection{Wind Turbine Operation}

Wind turbines generate electrical energy by extracting kinetic energy from the wind. Consequentially, downstream from the turbine, in the region referred to as the wake, the velocity is much lower than the flow regime upstream from the rotor. Turbine blades are an ensemble of smaller blade elements, each geometrically shaped with an airfoil profile, positioned at the optimal angle of attack with respect to the oncoming wind, for maximum lift generation. In order to maintain this optimal attack angle, the blade is twisted in the radial direction. The airfoil design enables lift generation on the blades, which sets them into rotational motion to extract kinetic energy from the wind. Figure 3.1 describes the parts of a horizontal axis wind turbine (HAWT). As shown in the figure, the roots of the blades are fastened to a central hub, connecting the blades to a shaft. The shaft, which is connected to a gearbox and generator, spins as the blades rotate, all of which is encased by the nacelle. The converted energy is then passed through ancillary services to be distributed to users. 


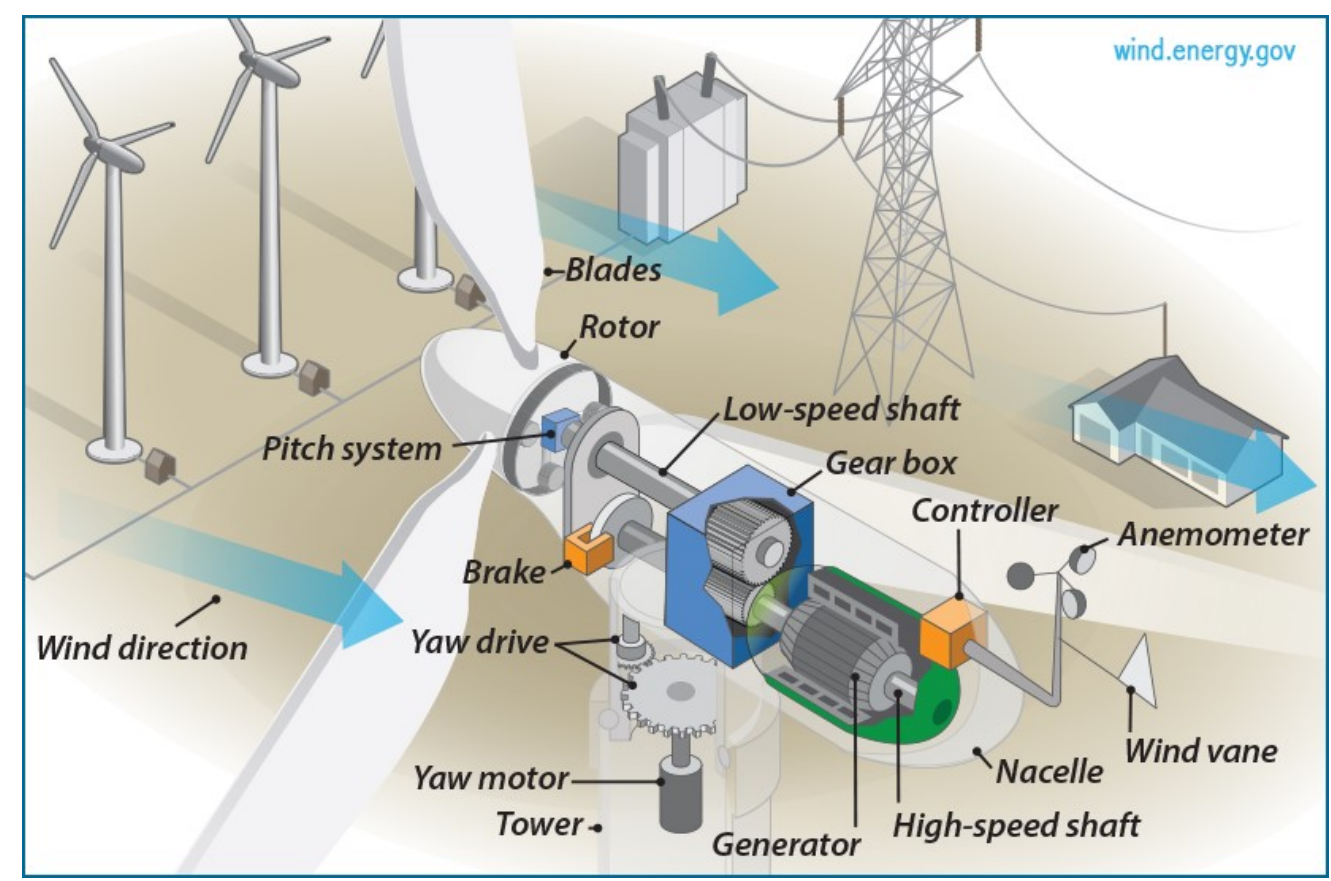

Figure 3.1 Wind Turbine Components [118]

\subsection{Lift Generation}

Understanding the fluid dynamics of lift generation is necessary to understanding the process of wake formation. Streamline curvature is fundamental to producing lift. For steady flows, particles travel along their own individual streamline to which their velocity vector is always tangent. Newton's Second Law can be applied to a particle by writing the particle acceleration in terms of the streamline coordinates, as shown in Equation 3.1 for the stream wise and normal directions. The variables $a_{s}$ and $a_{n}$ represent particle acceleration in the stream wise and normal directions respectively. The variable $U$, represents velocity, and $\mathrm{R}$ and $\mathrm{s}$ are the local radius of curvature of the streamline and distance along the streamline from an arbitrary initial point respectively [40].

$$
a_{s}=U \frac{\partial U}{\partial s}, a_{n}=\frac{U^{2}}{R}
$$


Prior to reaching the vicinity of the rotor disk, the flow regime is assumed to be at a constant atmospheric pressure, and the streamlines are assumed to be straight with an equal pressure above and below them. The wind flow is brought to rest at the tip of the blade's leading edge, referred to as the stagnation point. Above and below this point of zero-velocity, the streamlines encounter a negative, or favorable pressure gradient which enables the fluid to pass over the impeding blade. Shear stress induced from the fluid's viscosity, impedes the fluid's movement; however, the favorable pressure gradient over the blade's leading edge enables this effect to be overcome. Air is a viscous fluid, and has the tendency to stick to the blade wall. Boundary layer (BL) fluid travels around the curvature of the airfoil until a point of separation caused by an adverse pressure gradient. The adverse pressure gradient which is imposed by the outer surrounding flow, works against the boundary layer's forward motion and the flow eventually becomes separated from the blade surface. Implications of flow separation will be discussed in Section 3.2.1.

As streamlines follow the curvature of an airfoil, a difference in pressure is created between their top and bottom sides. When a fluid streamline is bent, a high pressure and low pressure side is created. Both sides of an airfoil are curved to positively contribute to lift through the direction they bend the streamlines in the inviscid region of the flow, outside the boundary layer. The changes in surface curvature cause changes in surface pressure. Figure 3.2 illustrates the pressure gradient normal to a curved surface [41]. 


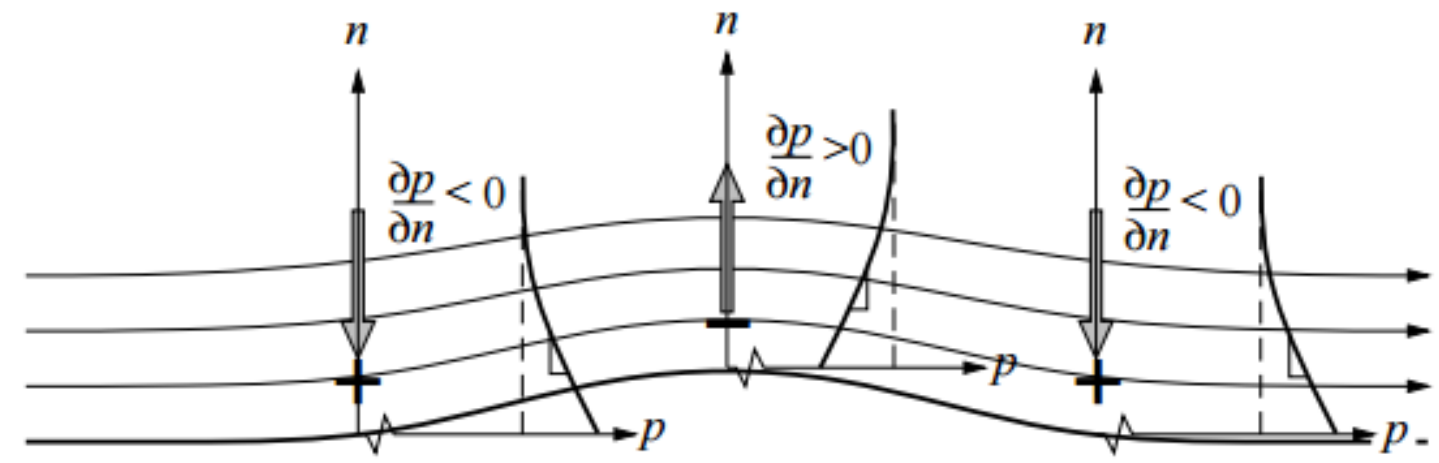

Figure 3.2 Pressure Distribution Normal to a Curved Surface

This variance in pressure, normal to a curved surface is defined by the normal-momentum equation, Equation 3.2, which is valid for inviscid flows at all Mach numbers. Equation 3.2 illustrates that a transverse pressure gradient, $\frac{\partial p}{\partial n}$, must exist to force fluid to flow along a curved streamline. The left hand side of the equation represents the variance in pressure, normal to the curved surface. Variables $\rho, V$ and $\kappa$ represent density, velocity and streamline curvature $(\kappa=$ $d \theta / d s)$ respectively [41].

$$
\frac{\partial p}{\partial n}=-\rho V^{2} \kappa
$$

The top surface of the airfoil bends the streamlines convexly, generating a relatively lower pressure towards the blade surface. On the bottom surface of the airfoil, the streamlines are deflected downwards, producing concave curvature in the streamlines which generates a higher pressure towards the blade surface. The net effect is a pressure imbalance between the top and bottom surfaces of the blade with the higher pressure side on the bottom, creating lift, the driving force of motion and consequentially leading to power generation of a wind turbine. This effect is visually represented in Figure 3.3. 


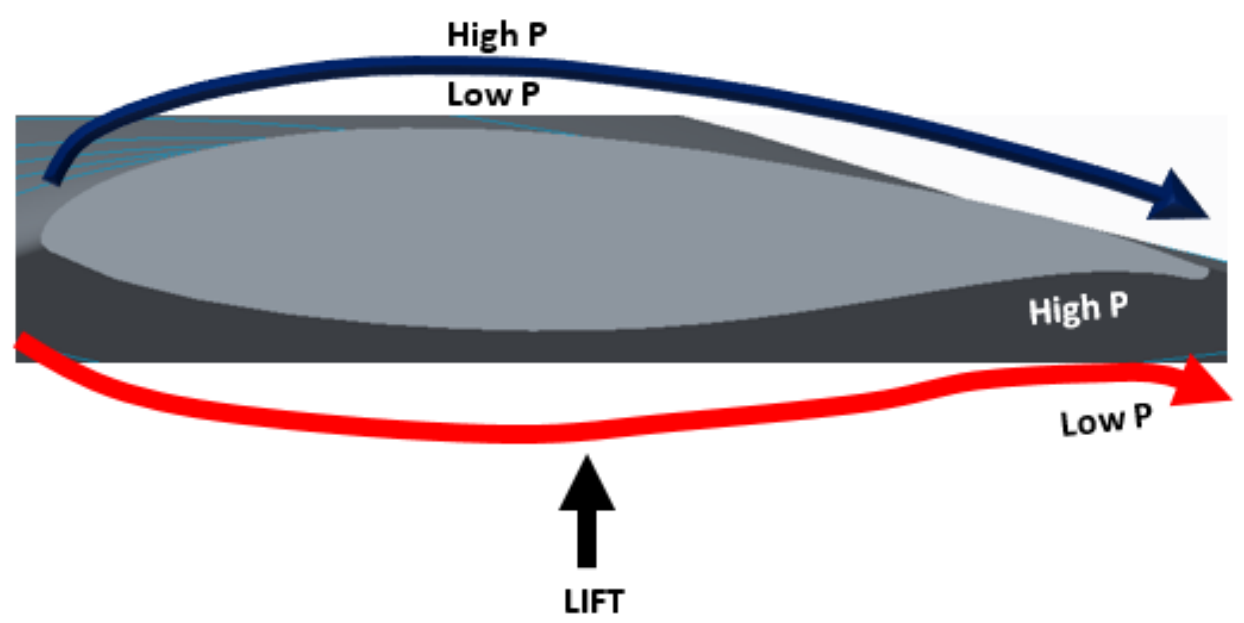

Figure 3.3 Lift on an Airfoil Due to Pressure Distribution

\subsubsection{Flow Separation}

As mentioned in Section 3.2, viscous properties of the air causes the air to "stick" to the blade surface. The viscosity causes the formation of a thin boundary layer enclosing the wall surface in which the velocity is quickly driven to zero towards the surface, a situation called the "no slip condition". A no-slip condition causes a velocity gradient normal to the wall surface to form which corresponds to the thickness of the boundary layer. This boundary layer velocity gradient creates distinct layers: a viscous sublayer followed by a buffer zone that turns into the logarithmic layer. After the logarithmic layer, the flow has become turbulent. Figure 3.4 relates the velocity gradient to the locations of the aforementioned sub layers of the boundary layer [42] 


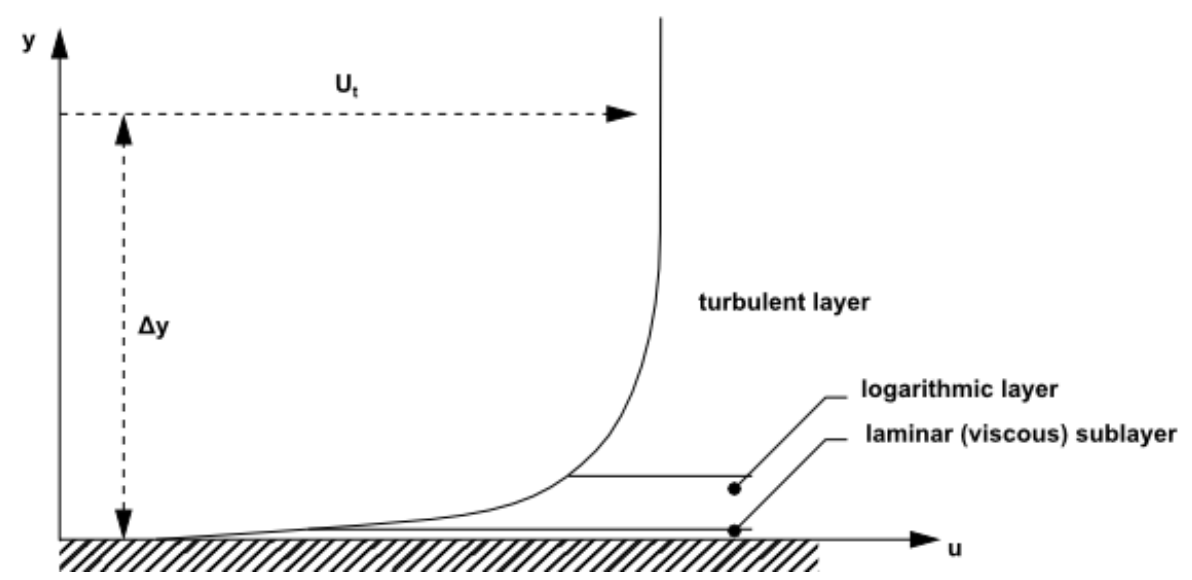

Figure 3.4 Regions of the Boundary Layer [42]

Viscous forces are formed from fluid viscosity and a velocity gradient, and are thus confined to the boundary layer [40]. There is a pressure differential working against the fluid's forward motion over the airfoil, having a stronger effect closer to the wall because the fluid's momentum is less in this region. The pressure of the external fluid streams causes interaction with boundary layer forces. These interactions increase the potential energy of the fluid, causing a decrease in kinetic energy, meaning the velocity of streamlines in the boundary layer are decreased. Eventually, the fluid slows to a point in which the inertia of the fluid disallows forward motion along the surface of the wall. The boundary layer's velocity slows to a point of nearly zero relative to the blade surface, and the shear stress is brought to zero. This point is called the separation point [43]. At the separation point, the shear stress becomes negative and some of the fluid begins to flow in the opposite direction, back towards the BL. As angle of attack is increased, the separation point occurs closer and closer to the leading edge of the airfoil. This occurrence is depicted in Figure 3.5 [44]. 

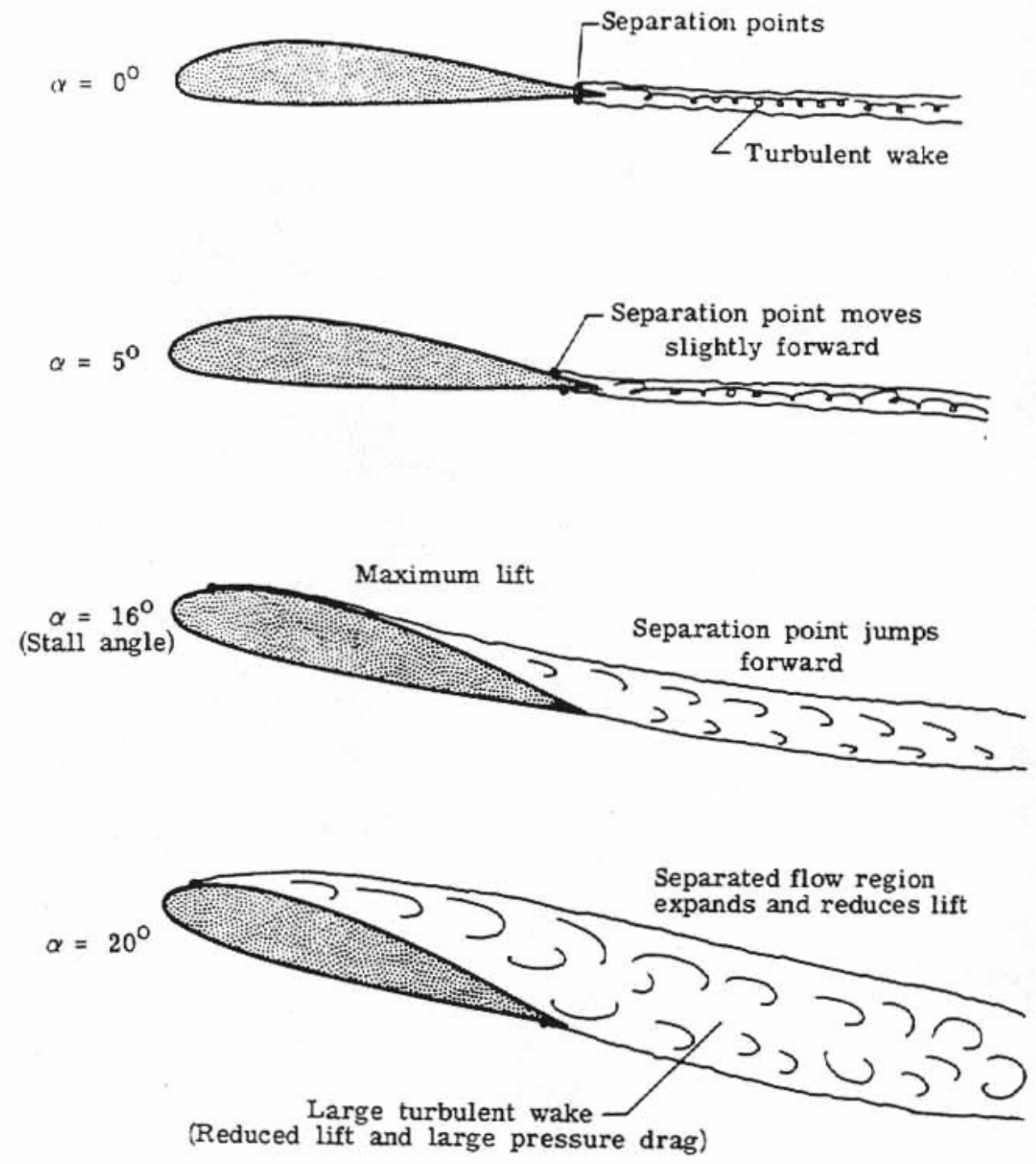

Figure 3.5 Boundary Layer Separation on an Airfoil [44]

The backflow, rolling up effect, results in counter-rotating vortex formation. This process continually occurs along the entire length of the blades, resulting in the continual shedding of vortex sheets, creating helical structures that travel downstream. This phenomenon generates a wake on the "departure" side of the airfoils, full of instabilities and disordered flow. Shed vortices eventually become stretched, resulting in merging of vortex elements in the wake which 
creates an intensification of vorticity [45]. Figure 3.6 is an image from a flow visualization experiment at the Royal Institute of Technology. The image depicts vortex shedding off the blade tips of a wind turbine [46]. Evidence of the vortex cores are distinct in the near wake region, and can still be sensed in the far wake region where they exist in a less distinct, but periodic fashion [47].

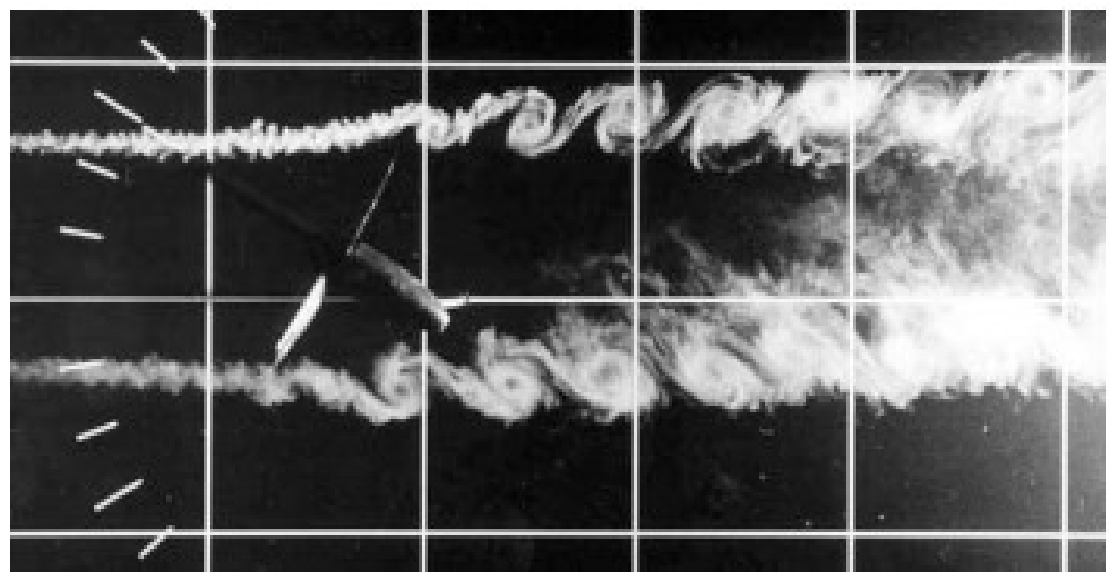

Figure 3.6 Flow Visualization of Vortex Shedding [46]

\subsubsection{Reynolds Number and Turbulence}

Higher Reynolds numbers are associated with raised levels of turbulence in a flow regime. Equation 3.3, the Reynolds number equation, shows that this number is based upon the ratio of inertial forces to the viscous forces where $U$ is the velocity, $c$ is the chord length and $v$ is the kinematic viscosity of the fluid.

$$
R e=\frac{U c}{v}
$$

In Section 3.2.1, the boundary layer was introduced and described as having distinct regions; the viscous sublayer, a transitional region and an inertial layer. Equation 3.3 indicates that Reynolds number is increased with velocity, showing that in high Reynolds number flows, inertial forces are dominant. This has a direct implication to the inertial layer of the boundary 
layer: as the Reynolds number increases, the inertial layer of the boundary layer will thicken [48].

Disorders in a flow field are caused by agitations from various sources such as atmospheric turbulence or the effect of blunt bodies in the flow. In Section 3.2.1, the occurrence of flow over a blunt body was discussed in the context of flow over a turbine blade, leading to vortex shedding. In low velocity flows, these agitations in the flow die down because they are dampened by the viscous forces which are dominant over the inertial forces. In high velocity flows, the inertial forces are dominant and they amplify the disturbances in the flow. Amplification of flow agitations in high velocity flows leads to strong mixing, as the motion of fluid particles becomes disordered. Through this disorder, the fluctuating velocity components of fluid particles interact with each other and bring about the transport of momentum, otherwise known as turbulent diffusion [45]. The effects of viscous damping and the amplification of flow agitations through inertial effects and their influence on the physical structures of eddies will be described in Section 3.2.3.

\subsubsection{Physical Structures in Turbulent flow}

Individual wakes are classified into a near wake zone which is $2-4$ rotor diameters downstream from the turbine, followed by a transitional zone, and a far wake zone beyond the transitional region. Within the near wake region, the blade configuration, as well as the pressure gradient along the axial frame of reference of the turbine, strongly influences the flow regime. In the far wake region, turbulence is the main contributor to the flow regime where large scale and small scale motions, called eddies, have evolved [49]. Eddies are swells of rotating flow, present in the far wake. In Section 3.2.1, the occurrence of vortex shedding is described. The vortex elements are very distinct in the near wake region, and become paired and stretched as they 
move downstream, evolving into eddies of varying length scales in the far wake region.

Figure 3.7 illustrates the progression of distinct vortices in the near wake regions to large scale structures in the far wake that form through the process of vortex pairing and stretching [50]. The largest length scale is represented by the variable $\mathrm{L}$, and the velocity of this length scale is represented by $V_{\text {Int. }}$. The variables used to represent the smallest length scale and its associated velocity are $\eta$ and $V_{K o l}$. respectively. The smallest eddy sizes can be on the order of the molecular size $(\sim 1 \mathrm{~mm}$ in the $\mathrm{ABL})$, referred to as the Kolmogorov scale, large eddy sizes are referred to as the integral scale $(\sim 1 \mathrm{~km}$ in the $\mathrm{ABL})[51]$. Both of these length scales can be mathematically described as discussed later in this section [45].

\section{Wake Structure}

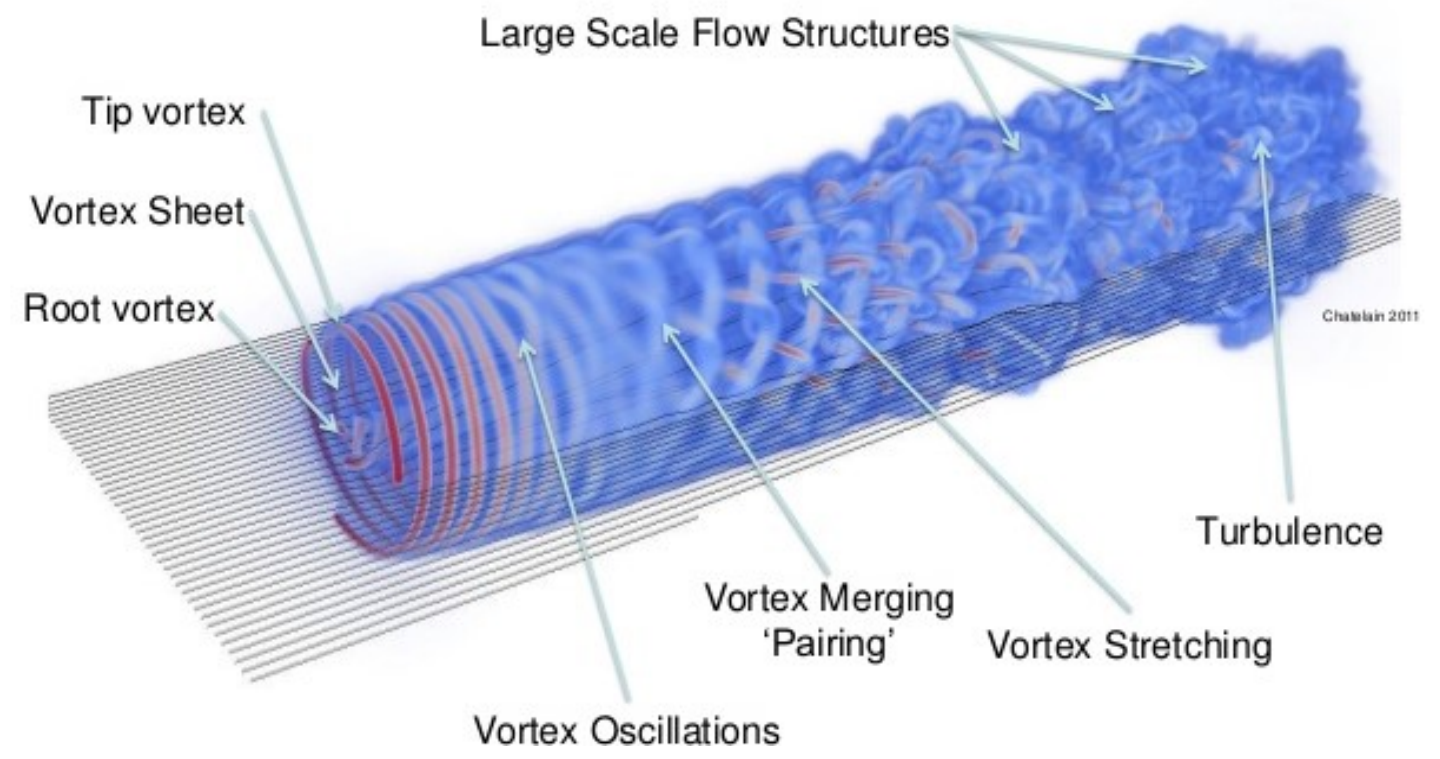

Figure 3.7 Evolution of a Wind Turbine Wake [50]

Large eddies are responsible for the majority of momentum and energy transfer between the freestream and wake flow. Due to the dominant inertial forces in high Reynolds number flow regimes, the kinetic energy that has been extracted by the large eddies from the mean flow cannot be dissipated through viscous forces. Once a large eddy extracts energy from the mean 
flow, it will evolve into smaller eddies. Energy extracted from the mean flow is subsequently passed on from larger eddies to smaller ones. Larger eddies contain a greater amount of energy. Kinetic energy extracted by large eddies from the mean flow cascades through the large eddies to eddies of diminishing length scales to a point in which it is eventually dissipated by the smallest eddies. Small eddies keep forming until the magnitude of the viscous force becomes on the order of the inertial force; viscosity controls the scale of the smallest eddies [51]. Turbulent kinetic energy that has been carried through eddies of decreasing sizes can be completely dissipated by the smallest eddies through viscous dissipation because the viscous effects are of equal or greater strength than the inertial forces. This process of extraction of turbulent kinetic energy to dissipation through eddies forms what is referred to as an energy cascade in the far wake region [49]. Figure 3.8 illustrates the energy cascade.

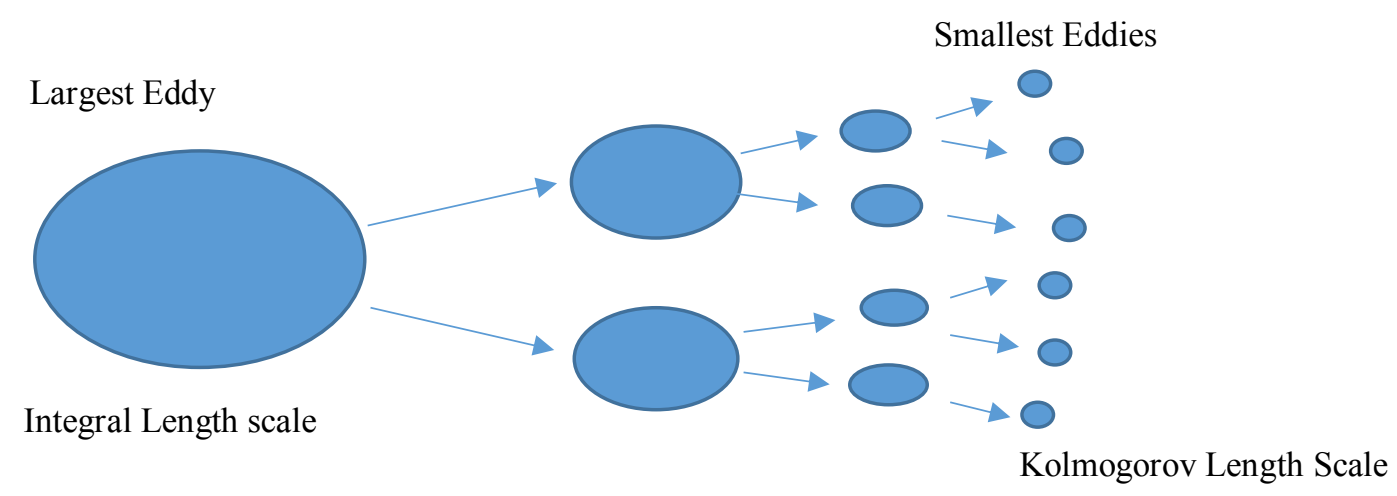

Figure 3.8 Energy Cascade

Theoretically, in statistically steady turbulent flow, the energy dissipated by the small scale eddies is equal to the energy supplied by the large scale eddies, and thus, no intermittent storage of energy occurs [52]. The balance between turbulent kinetic energy extracted from the mean flow and the energy that is dissipated, is used to formulate turbulence models. This will be described in Section 3.3.2.3. 
The rate of dissipation of turbulent kinetic energy, $\varepsilon$, is defined in Equation 3.4 where $v$ is the kinematic viscosity and $e_{i j}$ is a rate of deformation tensor. More specifically, the stress tensor $e_{i j}$, is the rate of deformation tensor for the smallest eddy and is given by Equation 3.5. The velocity is represented by the variable $V_{K o l}$. and the smallest eddy length scale, $\eta$, as the smallest eddy is responsible for dissipation [51] [45].

$$
\begin{gathered}
\varepsilon=v e_{i j} e_{i j} \\
e_{i j}=\frac{V_{K o l} .}{\eta}
\end{gathered}
$$

Where substituting Equation 3.5 into Equation 3.4 gives an alternate formula for kinetic energy dissipation, $\varepsilon$, in terms of the smallest eddy length scale, $\eta$, kinematic viscosity, $\nu$, and velocity, $V_{\text {Kol. }}$.

$$
\varepsilon=v\left(\frac{V_{K o l .}^{2}}{\eta^{2}}\right)
$$

The Kolmogorov length scale formula, Equation 3.7, defines the length scale, $\eta$ of the smallest eddies. Energy is dissipated at the Kolmogorov length scale, and this length scale depends on the dissipation rate, $\varepsilon$, and kinematic viscosity, $v$, rather than on the physical features, $V_{\text {Int. }}$ and L, of the largest eddies [51].

$$
\eta=\left(\frac{v^{3}}{\varepsilon}\right)^{1 / 4}
$$

Equation 3.7 is derived from the Reynolds number, knowing that for the length scale at which dissipation occurs, the viscous forces must be on the order of the inertial forces, therefore $R e_{\eta} \sim 1$. In terms of the length scale, velocity, and viscosity, this translates to Expression 3.8 which can be manipulated to mathematically define the smallest length scale in the turbulent flow field.

$$
\frac{V_{K o l . \eta}}{v} \sim 1
$$


Rearranging Expression 3.8 in terms of velocity and substituting this new form into Equation 3.6 eliminates the velocity term. After this substitution, Equation 3.6 can be rearranged, yielding Equation 3.7, defining the Kolmogorov length scale in terms of two variables. Once $\eta$ is found, the characteristic velocity for the smallest length scale can be solved for through Expression 3.8. The Kolmogorov length scale is used to determine the largest length scale, $L$, associated with the kinetic energy extraction rate, $k$. This is done through using the relationship between $k$ and $\varepsilon$ where the dissipation rate is on the order of the rate of turbulent kinetic energy extracted from the mean flow. First, the rate of turbulent kinetic energy extracted from the external flow is defined mathematically through Equation 3.9. The rate, $k$, is derived from the kinetic energy formula and is taken on a per unit mass basis. Large eddy velocity and length scales change as the eddies evolve over time, necessitating the use of a rate. The rate at which the large eddies change through the process of stretching and breaking up into smaller eddies is factored into the kinetic energy equation through multiplying by $\frac{1}{\text { timescale }}$, where timescale is $L / V_{\text {Int. }}$, yielding the expression:

$$
k=\frac{\frac{1}{2} * m * V_{\text {Int. }}^{2}}{m} \frac{V_{\text {Int. }}}{L}
$$

The simplified formula for $\mathrm{k}$ is shown through the proportionality Expression 3.10. This formula indicates that the large scale flow features are not influenced by viscosity, which is expected because of the dominant inertial forces at this scale.

$$
k \sim \frac{V_{\text {Int. }}{ }^{3}}{L}
$$


As stated in the above, the rate of the dissipation is on the order of the rate of turbulent kinetic energy extracted from the mean flow in the flow system, yielding the expression:

$$
k \sim \varepsilon
$$

This relationship can be used to relate the large eddy length scale, L, to the Kolmogorov scale, $\eta$. After substitution and several steps of algebraic manipulation, relating Equation 3.7 rearranged in terms of $\varepsilon$, with Expression 3.10 for $\mathrm{k}$ yields Expression 3.12.

$$
\frac{V_{\text {Int. }}{ }^{3}}{L} \sim \frac{v^{3}}{\eta^{4}}
$$

After algebraic manipulation shown in Appendix A, the ratio of large and small eddy length scales, $\mathrm{L}$ to $\eta$, is on the order of $\operatorname{Re}_{L}^{3 / 4}[45][51]$ :

$$
\frac{L}{\eta} \sim R e_{L}^{3 / 4}
$$

Expression 3.13 indicates that the large and small eddy length scales differ significantly, by a factor of the Reynolds number of the large scale flow structures. Difference in time scales, $t_{L}$ and $t_{\eta}$, between the large and small scale motions in the flow, is on the order of $R e_{L}{ }^{1 / 2}$ as shown in Expression 3.14. Timescale for the large scale eddies was defined earlier in this section and the small scale eddy time scale is $\sqrt{\frac{v}{\varepsilon}}[51]$.

$$
\frac{t_{L}}{t_{\eta}} \sim R e_{L}^{1 / 2}
$$

Time scales between the large and small-scale motions significantly differ by a factor of the Reynolds number for large scale motions. The ranges of length and times scales become greater as the Reynolds number of the large scale eddies increases.

Flow structures over the entire length and time scales, each possessing unique characteristics, have to be captured in CFD simulations in order to fully represent the energy 
cascade. The Navier-Stokes equations must be evaluated continuously over the entire length scale which in turn encompasses a wide range of time scales. In addition to a wide range of length and time scales, the unsteady, three-dimensional nature of turbulent flow creates an even more challenging scenario to simulate. Analytical simulations of turbulent flows are not possible; however, approximation techniques using statistical models are feasible and are described in Section 3.3.2.3.

As wakes develop behind a wind turbine, a shear layer is formed from the difference in velocity between the inside low velocity region of the wake and the outside freestream velocity. The wake in the velocity contour plot of Figure 3.9 is indicative of the presence of a shear layer through the distinct color transition from the freestream towards the inside of the wake. This figure is a top-down view of two wind turbines from an early simulation of this research.

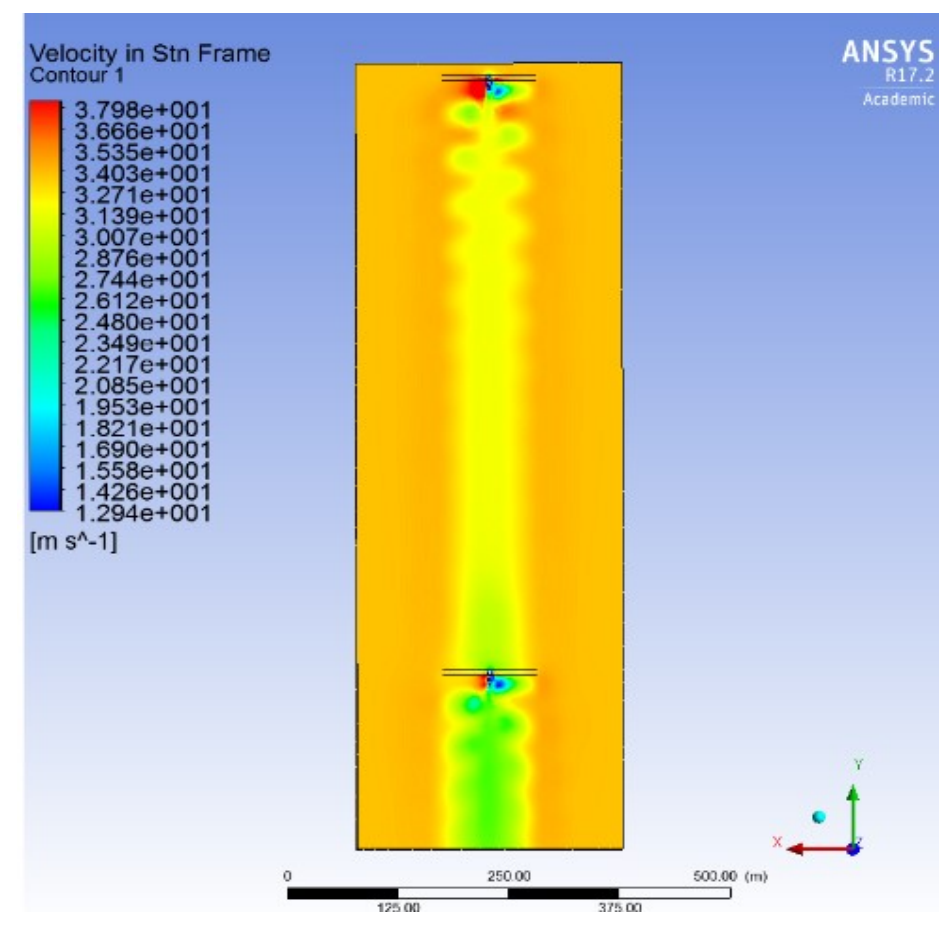

Figure 3.9 Wake Contour Plot

Eddies are induced from shear stress, and the eddies form within the shear layer. The shear layer thickens in the far wake region, where large and small scale motions have formed [53]. 
Large scale motions can cause wake meandering, which is the lateral movement of an entire wake. Wake meandering effects have the potential to cause extreme loads, especially yaw loads on turbines as the wake drifts in and out of the rotor planes of downstream turbines. Meandering can also diminish the overall deficit of a wake to ultimately ease power losses [53]. Early research on wake meandering demonstrated that meandering has a significant impact on reducing the magnitude of wake deficits [54].

Wake dissipation occurs through mixing between the low velocity fluid inside the wake and the freestream fluid of a higher velocity. The main catalyst of this mixing is the large scale eddies that have formed. Through this process, high velocity fluid from outside the wake is transferred into the wake, causing wake expansion, reducing the velocity deficit [53], [55]. Mixing transfers momentum and energy from the higher energy freestream region to the low energy flow within the wake, which causes the wake fluid to eventually recover to the freestream velocity.

\subsection{Wake Simulation through CFD}

CFD simulations use mathematical models grounded in conservation principles; namely the conservation of mass, momentum and energy to predict the behavior of a flow regime. Application of these principles allows for the equations of fluid motion, the Navier-Stokes equations to be resolved [48]. The Cartesian form of the Navier-Stokes equations, given in Equations 3.15-3.18 define the motion of any Newtonian flow. The variables $\rho, t, p$, $\mu$ represent density, time, pressure and viscosity respectively. The source term is represented by $S_{x, y, z}$. Source terms in turbulent flow are imposed through vorticity [45]. The variables $x, y, z$ represent the three dimensions in space, and the Cartesian system is represented by $u, v$ and $w$. 


$$
\begin{gathered}
\frac{\partial u}{\partial x}+\frac{\partial v}{\partial y}+\frac{\partial w}{\partial z}=0 \\
\rho\left(\frac{\partial u}{\partial t}+u \frac{\partial u}{\partial x}+v \frac{\partial v}{\partial y}+w \frac{\partial w}{\partial z}\right)=-\frac{\partial p}{\partial x}+\mu\left(\frac{\partial^{2} u}{\partial x^{2}}+\frac{\partial^{2} u}{\partial y^{2}}+\frac{\partial^{2} u}{\partial z^{2}}\right)+S_{x} \\
\rho\left(\frac{\partial v}{\partial t}+u \frac{\partial v}{\partial x}+v \frac{\partial v}{\partial y}+w \frac{\partial v}{\partial z}\right)=-\frac{\partial p}{\partial y}+\mu\left(\frac{\partial^{2} v}{\partial x^{2}}+\frac{\partial^{2} v}{\partial y^{2}}+\frac{\partial^{2} v}{\partial z^{2}}\right)+S_{y} \\
\rho\left(\frac{\partial w}{\partial t}+u \frac{\partial w}{\partial x}+v \frac{\partial w}{\partial y}+w \frac{\partial w}{\partial z}\right)=-\frac{\partial p}{\partial z}+\mu\left(\frac{\partial^{2} w}{\partial x^{2}}+\frac{\partial^{2} w}{\partial y^{2}}+\frac{\partial^{2} w}{\partial z^{2}}\right)+S_{z}
\end{gathered}
$$

These equations are difficult to solve as they are highly sensitive to initial conditions and must address a wide range of length scales present in the flow as described in Section 3.2.3. As stated in Section 3.1, wakes are regions of turbulent flow, a flow regime that appears random. This seemingly random behavior in turbulent flow can actually be described in terms of time and space; and simulated through the Navier-Stokes equations. Analytical solutions are only possible for very simple problems because of the complexity of the Navier-Stokes equations. Instead, the equations are simplified through assumptions as described in Section 3.3.1. Several methods have been derived which approximate a representation of the Navier-Stokes equations. The simplification techniques and governing equations used to represent the Navier-

Stokes equations are described in Section 3.3.1 and Section 3.3.2 respectively.

\subsubsection{Simplifications to Fluid Equations}

Numerical solutions evaluate the motion of flow by subdividing the continuous problem into discrete points in the domain. The quality of discretization used, greatly impacts the precision of the numerical solution [56]. Simulations aim to imitate the appearance of a physical real-life scenario. CFD uses many mathematical models to simulate turbulent flow. These mathematical models are simplified descriptions of the actual physical flow scenario. The nature of this work 
requires certain assumptions to be made as the complexities of turbulent flow, such as rapidly fluctuating flow parameters and stretching of vortex elements are continuously occurring. Simplifications have the ability to greatly reduce computational expense. Assumptions made to simplify the equations also have the potential to skew the results. The assumptions are made based upon their significance to the overall results. The sensitivity of the simulation results to certain parameters such as time step and grid size is analyzed in comprehensive CFD studies. If reducing grid density and increasing time step does not change the overall results, the overall computational expense of the simulation can be reduced. Sections 5.2.1 and 5.3.4 describe the grid and time step sensitivity studies performed for the offshore array simulations of this work. Further simplifications in the simulation can also be made simplifying the geometric representation of the rotor, as described in Section 4.2. Also, statistically averaging the NavierStokes equations is an approximation technique employed by CFD to enable complex flow patterns to be simulated. The mathematics of this technique is described through the governing equations in Section 3.3.2.

To reduce the complexities of the flow equations, certain assumptions can be made regarding the physicalities of the flow. The fluid problem to be solved in this research is assumed to be incompressible as the Mach number is below 0.3 with the highest speed, $70 \mathrm{~m} / \mathrm{s}$, reached at blade tips. Recent designs are introducing larger rotor diameters, such as the National Renewable Energy Lab (NREL) 5MW offshore wind turbine which has blade lengths of $90 \mathrm{~m}$. For a $200 \mathrm{~m}$ diameter turbine rotating at $25 \mathrm{rpm}$, the Mach number at the blade tips will be approximately 0.78, large enough for compressibility to take effect [57]. Incompressibility allows the assumption that density remains constant throughout the domain. 
Another simplification that can be made to complex flow modelling is through using streamlines to represent the path that a fluid particle takes. Also, assumptions about viscosity can be made for further simplification. In regions a significant distance from the blades and other turbine parts, viscosity can be considered a negligible force, thus the flow calculations are greatly simplified [58]. Within the boundary layer, viscous forces are very important and change the trajectory of the fluid particles, altering the streamlines and giving them a curvature.

Consequentially, viscous effects must be included in the boundary layer regions of the flow field. Figure 3.10 indicates the locations of the assumed inviscid and viscous regions around an airfoil [59] .

Inviscid region

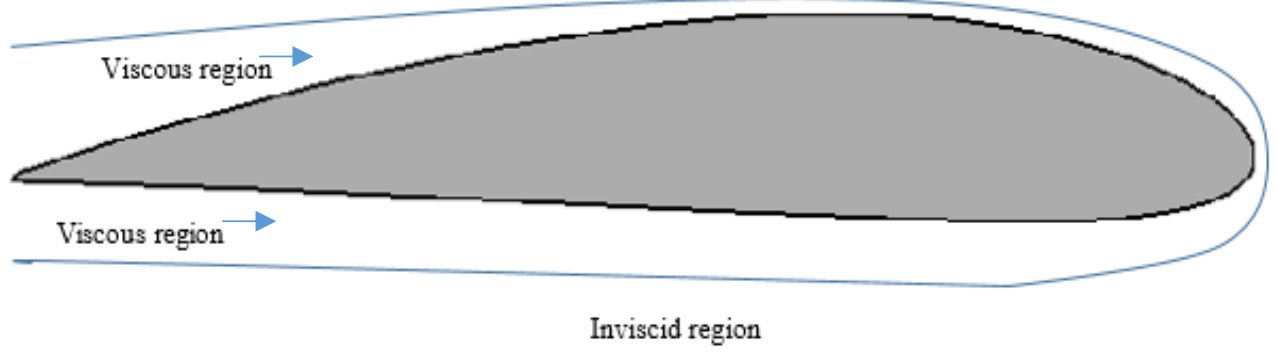

Figure 3.10 Regions of Flow Surrounding an Airfoil

\subsubsection{Approximating the Navier-Stokes Equations}

As described in Section 3.1, transport of turbulent flow displays a chaotic behavior where parameters of velocity and pressure rapidly fluctuate, making the flow problem difficult to be resolved analytically. Analytical solutions are exact solutions to a problem, whereas numerical solutions are approximations, solved through numerical techniques. Complex flow regimes in the natural world cannot be solved analytically unless they are extremely simple and under ideal conditions. Numerical methods must be employed to solve algebraic approximations of the 
Navier-Stokes. The four most common CFD techniques employed to approximate the NavierStokes equations for turbulent flow are: Direct Numerical Simulation (DNS) using the NavierStokes equations, Large Eddy Simulation (LES) using filtered Navier-Stokes equations, Detached Eddy Simulation (DES) and the Reynolds-Averaged Navier-Stokes equations (RANS) modelling technique. These different techniques are unique from each other in the way they model and or resolve turbulence [60].

\subsubsection{Common Four CFD Techniques}

DNS resolves all eddy length scales of the turbulent flow using the Navier-Stokes equations. Accuracy is high in DNS as the method resolves the Navier-Stokes equations for the entire length scale of the flow regime without using averaging techniques that are used in alternate methods such as RANS. DNS requires an extremely fine mesh and small time steps in order to capture the smallest motions. This method can only be used for simple, low Reynolds number flow problems as the grid requirements exceed current computational resources [48] [61].

In LES, only the large eddy behaviors are resolved. Small scale motions are filtered out before resolving the unsteady Navier-Stokes equations. To include the effects of the small scale motions that are left unresolved, an additional model, the sub-grid-scale (SGS) model, is employed to resolve the stresses in the flow, associated with the smaller eddies [45]. LES is an attractive technique as the method can handle extremely separated flow problems, making LES superior to RANS for modelling turbulent flow [48] [62]. LES can also handle complex geometry well [45]. The drawback is that LES is very computationally expensive and requires a Courant-FriedrichsLewy (CFL) condition of 1 for stability, a condition described in Section 5.3.4. This condition requires that the time step be very small in addition to an extremely fine grid required to fully capture all length scales. The region of highest computational complexity in LES is near the 
wall, where the grid is significantly finer than other regions of the flow domain. DES uses LES methodology in the majority of the domain but attempts to reduce the computational complexity in the near wall region by switching to the simpler solving technique used in RANS. Turbulence length scales or user defined controls signals the solver to switch from LES to RANS modelling [62].

The turbulence energy spectrum in wave number space is used to define the turbulence of a flow where the wave number $\mathrm{k}$, is equal to $2 \pi$ /wavelength. Smaller scale motions carry most of the Reynolds stresses and the larger scale motions carry most of the kinetic energy [56]. DNS, LES and DES are much more computationally expensive than RANS because they resolve small scale motions. Figure 3.11 is a graphical representation of the turbulence energy spectrum [51]. Labels on the graph indicate the eddy scales that the aforementioned CFD solving techniques model and resolve [56].

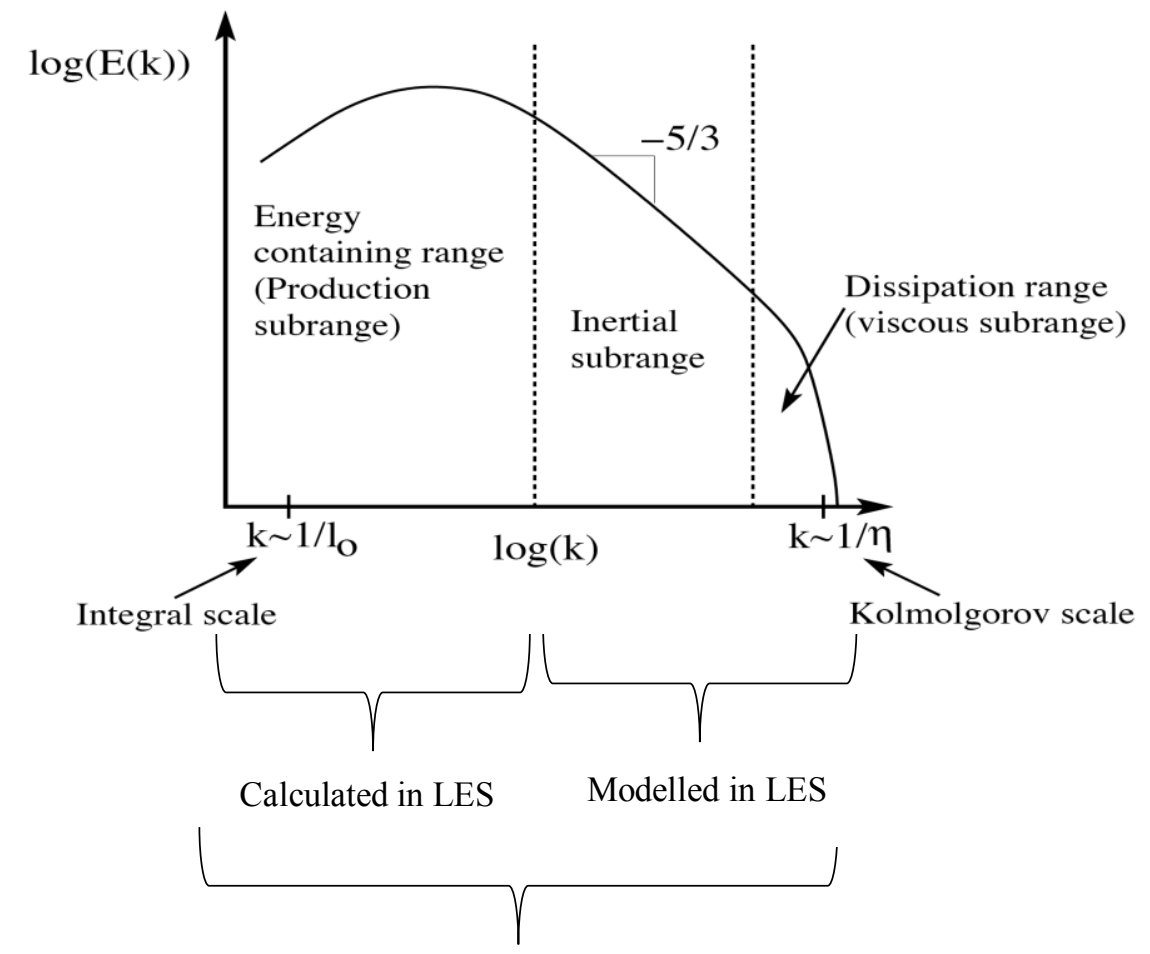

Calculated in DNS. modelled in RANS

Figure 3.11 Simulation Capacity of the Main Turbulence Models [51] 


\subsubsection{Reynolds Average Navier-Stokes (RANS)}

RANS is an attractive model for CFD simulations as the method can resolve the Navier-Stokes equations within reasonable computational expense. This method was selected for the simulations of this research and is described in greater detail than the alternative models mentioned above. To approximate the Navier-Stokes equations through RANS, statistics are used to approximate the flow where the instantaneous flow quantities are calculated by the sum of a mean component and a fluctuating component that is brought about by vortices. This approximation concept is shown in Equations 3.19 and 3.20 for the $i$-direction of velocity and pressure respectively.

$$
\begin{gathered}
U_{i}=\bar{U}_{\imath}+U_{i}^{\prime} \\
P_{i}=\bar{P}_{\imath}+P_{i}^{\prime}
\end{gathered}
$$

Equations 3.19 and 3.20 can be written in proper form for all three dimensions. The next step in obtaining the RANS flow equations is to substitute the appropriate forms of Equation 3.19 and 3.20 into the continuity and momentum equations (Equations 3.15-3.18) and time averaging each modified equation. Lastly, the Reynolds operator is employed (Equation 3.21) which states that the average of the fluctuation is equal to zero.

$$
\overline{U_{l}{ }^{\prime}}=0
$$

This yields the governing equations of RANS which can be used to obtain a solution for the mean flow. The continuity equation is displayed in Equation 3.22 and the $i$-direction Reynolds Average Navier-Stokes equation is shown in Equation 3.23 [63] [45].

$$
\begin{gathered}
\frac{\partial \overline{U_{i}}}{\partial x_{i}}=0 \\
\rho\left(\frac{\partial \overline{U_{l}}}{\partial t}+\frac{\partial}{\partial x_{j}}\left(\bar{U}_{\imath} \bar{U}_{J}\right)=-\frac{\partial \bar{p}}{\partial x_{i}}+\frac{\partial}{\partial x_{j}}\left(\mu \frac{\partial \overline{U_{l}}}{\partial x_{j}}\right)-\frac{\partial}{\partial x_{j}}\left(\overline{\rho U_{l}^{\prime} U_{J}^{\prime}}\right)\right.
\end{gathered}
$$


The $U$ terms represent the flow's velocity, $p$ is pressure, $\mu$ is viscosity and $-\overline{\rho U_{l}^{\prime} U_{J}^{\prime}}$ represents the Reynolds stresses [63]. The Reynolds stresses are additional terms added to the NavierStokes equation through the averaging process. In Equation 3.23 the units of stress, attached to the $\overline{\rho U_{l}^{\prime} U_{j}^{\prime}}$ term, are apparent. Fluctuations of $U$ in the $i$-direction can interact with fluctuations

of $U$ in the $j$-direction to create momentum transfer. Through this process, turbulent stress arises [45]. The Reynolds stresses act in all dimensions, and introduce 6 additional unknowns into the system of equations. Turbulence models have been developed to close the RANS equations by formulating equations to compute the Reynolds stresses. The turbulence models used for closure of the RANS equations are described in Section 3.3.2.3.

\subsubsection{Turbulence Models}

In Section 3.3.2.2, RANS was described. Through the process of substituting an average and fluctuating component for velocity in the Navier-Stokes equations and time averaging these equations, unknown variables called the Reynolds stresses were introduced. Turbulence models have been formulated to close the RANS equations by solving for the Reynolds stresses. Two main categories of turbulence models exist: eddy viscosity models and Reynolds stress transport models (RSM) [45] [60]. Several eddy viscosity models exist, they differ in their amount of equations as well as overall accuracy and applicability to certain flow regimes. Table 3.1 provides a breakdown of the classes of turbulence models. 
Table 3.1 Classification of Prominent Turbulence Models

\begin{tabular}{|c|c|c|}
\hline Number of Equations & Eddy Viscosity & $\begin{array}{c}\text { Reynolds Stress Model } \\
\text { (RSM) }\end{array}$ \\
\hline $\mathbf{0}$ & - Mixing Length & {$[-]$} \\
\hline 1 & $\begin{array}{ll}\text { - } & \text { Prandtl } \\
\text { - } & \text { Spalart-Allmaras }\end{array}$ & {$[-]$} \\
\hline 2 & $\begin{array}{ll}\text { - } & k-\varepsilon \\
\text { - } & k-\omega \\
\text { - } & k-\omega \text { SST }\end{array}$ & {$[-]$} \\
\hline 7 & {$[-]$} & RSM \\
\hline
\end{tabular}

Zero equation (algebraic) models require the least amount of computational expense compared to other turbulence models but cannot be applied to turbulent flows with vortex shedding. This is because the variation in length scales is hard to algebraically define [64]. Prandtl's mixing length model, developed in 1925, simulates Reynolds stress momentum transfer by computing eddyviscosity. Eddy-viscosity, $\mu_{T}$, in this model relies on a simple equation, Equation 3.24, which requires an input of the mixing length, $l_{\text {mix }}$. The mixing length is a user-defined value based on empirical values which are specific to the nature of the flow [48].

$$
\mu_{T}=\rho l_{m i x}{ }^{2} \frac{d \bar{u}}{d y}
$$

One equation models resolve eddy-viscosity through a single partial differential equation. Common one-equation models are the Prandtl model and Spalart-Allmaras model. Prandtl's model is the simpler of the two, only requiring two closure coefficients while the Spalart- 
Allmaras model requires seven [48] [64]. They are more extensive than the algebraic model, however, they are unable to accurately simulate complex turbulent flow.

The mixing length model is improved upon through two equation models by including turbulent kinetic energy and dissipation in their formulation. Two equation models consist of a turbulent kinetic energy equation and an equation for the turbulent length scale. The main two equation models are the $\mathrm{k}-\varepsilon, \mathrm{k}-\omega$, and $\mathrm{k}-\omega$ shear stress transport (SST) models. The k- $\varepsilon$ model, typically accepted as the default model in CFD, is a significant improvement upon the one equation models, capable of simulating a wider range of flow regimes. A significant drawback of the $\mathrm{k}-\varepsilon$ model is an over-prediction of shear-stress magnitudes which causes the model to produce a solution with a delayed separation point, or in some cases prevents separation altogether. This insensitivity to adverse-pressure gradients makes the model less desirable for separated flow.

The k- $\omega$ model, developed by Wilcox improved upon the shortcoming of the k- $\varepsilon$ model but the eddy-viscosity calculation in this formulation is very sensitive to the flow values of the freestream. The k- $\omega$ SST is a further improvement over the k- $\omega$ model [64]. This model uses the $\mathrm{k}-\omega$ model to simulate the near wake region, then uses a blending function to switch to the $\mathrm{k}-\varepsilon$ model in freestream regions of the flow where shear is not present, avoiding the aforementioned sensitivity of the standard $\mathrm{k}-\omega$ model to freestream conditions. This model performs well for flow separation; both in the prediction of onset and amount in adverse pressure gradients. Increased performance in simulating separation is due to k- $\omega$ SST's inclusion of transport effects in the eddy-viscosity calculation. A shortcoming of this model is that the model assumes isotropic turbulence, which is not always the case. In the largest region of the boundary layer, turbulent kinetic energy is greater than dissipation [48]. 
Near wall treatment is also an important aspect of turbulence modelling. The near wall treatment varies between the k- $\omega$ and k- $\varepsilon$ models. As discussed in Section 3.2.1, the boundary layer consists of three regions. In order to fully capture the boundary layer flow in simulation, the grid point nearest to the wall must be located in the viscous sublayer when using the Low-Reynolds-Number method. This can be very difficult to achieve in some cases due to computational power constraints. To reduce computational expense while still producing acceptable solutions, the automatic wall treatment feature was developed by ANSYS CFX. In this treatment, an automatic switch from the low-Reynolds method to wall functions occurs when necessary [42]. The automatic near-wall treatment is applied by the $k-\omega$, and $k-\omega$ SST models.

Wall functions approximate portions of, or all boundary layer sub layers. Aside from the necessary scaling factors, wall functions assume the formulation defining the wall to outer edge of the logarithmic layer boundary layer region to be of the same form for all near wall flow scenarios [65] . The most basic, earliest wall functions assumed the nodes nearest to the wall to be located outside the viscous sublayer. This methodology yields inaccurate solutions if the mesh is fine enough to have the nearest nodes to the wall within the viscous sublayer [66]. Scalable wall functions, which are employed in ANSYS CFX, have overcome this barrier and can be used with arbitrarily-fine meshes. These wall functions are applied in the $k-\varepsilon$ model [67]. Automatic wall functions employed by $\omega$-based models switch to wall functions in near-wall regions with coarse meshes. This function allows for arbitrarily fine meshes to be used near the wall, ultimately reducing computation time. The main difference in near wall treatments for $\varepsilon$-based and $\omega$-based models is the way in which they model the viscous sublayer. An analytical expression is used to resolve the viscous sublayer in $\omega$-based models and the logarithmic layer is approximated [66]. This is not the case for $\varepsilon$-based models. No analytical expressions are used for resolving the viscous 
sublayer in $\varepsilon$-based models and the entire region of the boundary layer is approximated [48]. The $k$ - $\omega$ SST model was implemented by the simulations of this research and the model is further discussed in Section 5.2.2.

Reynolds-stress models differ from eddy-viscosity models by including additional equations to account for the transport of the principle shear stress. All six Reynolds stresses are solved through an additional transport equation. The seventh equation is included for calculation of turbulent dissipation [45]. Inclusion of this term has enabled more accurate simulations for flow regimes with adverse pressure gradients than eddy-viscosity models [64], [48]. The cost of improved accuracy is an increase in computational expense [48]. Table 3.2 summarizes the information presented above for ease of comparison. 
Table 3.2 Turbulence Model Comparison Chart

\begin{tabular}{|c|c|c|c|c|}
\hline ZERO EQUATION & \multicolumn{2}{|l|}{ Prandtl } & \multicolumn{2}{|c|}{ Spalart-Allmaras } \\
\hline Applicability & \multicolumn{2}{|c|}{$\begin{array}{l}\text {-Incomplete as it assumes the } \\
\text { turbulence length scale is } \\
\text { directly related to a flow } \\
\text { parameter }\end{array}$} & \multicolumn{2}{|c|}{$\begin{array}{l}\text {-Improved prediction of free } \\
\text { shear flow } \\
\text {-More accurate velocity } \\
\text { profiles } \\
\text {-Unsuitable for adverse } \\
\text { pressure gradients }\end{array}$} \\
\hline Computational Expense & \multicolumn{2}{|l|}{ - Inexpensive } & \multicolumn{2}{|c|}{ - Inexpensive } \\
\hline ONE EQUATION & \multicolumn{4}{|c|}{ Mixing Length } \\
\hline Applicability & \multicolumn{4}{|c|}{$\begin{array}{l}\text { - Only applicable to simple flow problems } \\
\text { - Cannot handle rotational flow or separation } \\
\text { - Relies on empirically defined mixing lengths and is therefore } \\
\text { incomplete }\end{array}$} \\
\hline Computational Expense & \multicolumn{4}{|l|}{ - Inexpensive } \\
\hline TWO EQUATION & k- $-\varepsilon$ & \multicolumn{2}{|l|}{ k- $\omega$} & k- $\omega$ SST \\
\hline Applicability & $\begin{array}{l}\text { - Poorly predicts } \\
\text { rotating and highly } \\
\text { separated flows } \\
\text { - Approximates the } \\
\text { whole boundary } \\
\text { layer }\end{array}$ & \multicolumn{2}{|c|}{$\begin{array}{l}\text { - Better } \\
\text { performance for } \\
\text { separated flows } \\
\text { than the k- } \varepsilon \text { model } \\
\text { - Higher accuracy } \\
\text { near walls than the } \\
\text { k- } \varepsilon \text { model } \\
\text { - Overly sensitive } \\
\text { to the external flow } \\
\text { velocity at the BL } \\
\text { edge }\end{array}$} & 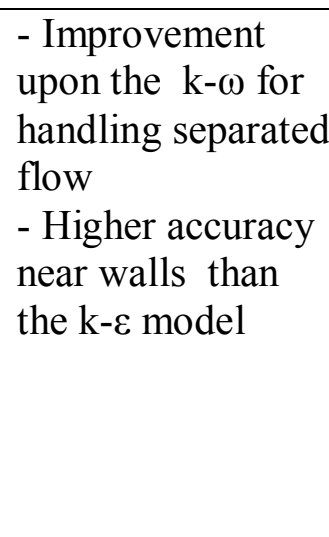 \\
\hline Computational Expense & $\begin{array}{l}\text { - Higher than zero- } \\
\text { equation model }\end{array}$ & \multicolumn{2}{|c|}{$\begin{array}{l}\text { - More detail than } \\
\mathrm{k}-\varepsilon\end{array}$} & $\begin{array}{l}\text {-Small increase } \\
\text { from } \mathrm{k}-\omega\end{array}$ \\
\hline SEVEN EQUATION & \multicolumn{4}{|c|}{ RSM } \\
\hline Applicability & \multicolumn{4}{|c|}{$\begin{array}{l}\text { - Accurately simulates flow rotation and swirl } \\
\text { - Accounts for streamline curvature }\end{array}$} \\
\hline Computational Expense & \multicolumn{4}{|c|}{ - Very expensive (2-3 times greater than alternative models) } \\
\hline
\end{tabular}




\subsubsection{Solver Control in CFD}

The nature of the flow regime in the research which includes the behavior of vortex shedding, requires the use of transient models during the simulations. In ANSYS CFX, the simulation can be run with either a steady-state or transient solving scheme. In CFX's steady-state models, a pseudo-transient technique is imposed, where a false time step is applied to under-relax the equations. Large time steps can be used as the solving scheme is fully implicit. Convergence in the steady state model is reached very quickly compared to the transient approach [68]. In steady state scenarios, the velocity and pressure parameters do not change with time. Steady state simulations do not require information related to simulation duration to be solved; the assumption is made that the steady conditions are reached after a relatively long period of time. Transient simulations do require user inputs regarding simulation duration to determine the time intervals at which the flow equations are to be solved at. In transient simulations, the parameters of velocity and pressure fluctuate with time to account for the unsteady nature of the flow in the simulation. At each time step, the governing equations are evaluated to compute a solution for all nodes in the fluid domain [69], [70]. Virtually all flows are transient in nature, a steady state assumption can only be made if the transient fluctuations are ignored and a time-averaging method is imposed [71]. The solution of a simulation run with the steady-state models can be used as an initial guess for the transient simulation for a particular flow problem to reduce computation time as this guess is expected to be much more accurate and realistic than the automatic initial guess by the solver. Eventually after sufficient run time, a transient simulation will progress towards a steady-state or time-periodic solution. In steady-state solutions, the flow parameters stop changing over time. For a time-periodic solution, the flow parameters will oscillate in a repeating pattern [72]. 
Transient solving schemes involve solving derivatives with respect to time using either a firstorder accurate scheme, the First Order Backward Euler method, or a second-order accurate scheme called the Second Order Backward Euler method. These implicit methods are commonly used in numerical solvers to solve the ordinary differential equations. The discontinuous Galerkin method can be used in CFD to evaluate the Euler equations. Information on the formulation of the aforementioned methods can be found in [73].

Before resolving the flow, the fluid domain is discretized into finite volumes, which is done by creating a mesh. Each finite volume is a control volume that surrounds a node. Nodes, the mesh vertices, are storage locations for all of the fluid properties and solution values. Over each control volume, the conservation of mass and momentum equations and a passive scalar are integrated rather than evaluating the differential form of the flow equations [48] [74]. As described in Section 3.3, fluid flow problems are most often nonlinear and cannot be solved analytically. Therefore, in order to resolve the discrete system of linearized equations, ANSYS CFX uses an iterative process called the Multigrid accelerated Incomplete Lower Upper factorization technique [75]. Through this process, the final solution is obtained over many iterations. To monitor the accuracy of the solver's iterations, residuals are calculated for the three-dimensional governing Navier-Stokes equations, the continuity equations, and the turbulence model equations. For each control volume, conservation equations are formulated and the local imbalance of these equations, the residual, is computed. Residual values are output for each of the aforementioned equations for every control volume in the fluid domain, at every time step. In ANSYS CFX, the root mean square technique is employed to normalize the solution residuals. As the solver progresses through time, the magnitude of the residuals should decrease if a proper mesh and accurate boundary conditions are employed in the set up phase. If 
this is the case, the solutions are said to be converging. Relatively loosely converged solution would have RMS residual levels of 1E-4. This is a sufficient level of convergence for many applications. Values of 1E-5 are considered well converged and values of 1 E-6 are said to be tightly converged [76]. Domain imbalance is also monitored for solution accuracy. Imbalance quantifies the conservation of mass, momentum and energy over the entire domain. In a converged solution, the total flux entering the domain should be the same as the total flux exiting the domain (i.e. less than 1\%). Defining the time step is also a requirement for transient simulations. ANSYS documentation recommends that the inverse of the angular velocity of the rotating machine be used for the time step [77]. 


\section{Literature review}

Computational Fluid Dynamics (CFD) is a computer simulation tool which characterizes fluid motion through partial differential equations. CFD allows for a representation of very large scale complex flow regimes to be analyzed in detail which would otherwise be extremely difficult and often impossible to be solved analytically. The study of cumulative wake effects over a marine environment is difficult to simulate because flow problems are by the majority, complex and highly nonlinear and so very rarely can the Navier-Stokes equations be solved analytically. In this research, CFD was employed to simulate wakes of clusters of offshore wind turbines. A review of prominent work for simulating wind farm wakes and a review of techniques for rotor geometry and boundary condition representation is included in Section 4.2. Another component of the research was to validate CFD simulation techniques with a small scale wind tunnel test case. A brief review of wind turbine experimentation in wind tunnels is included in Section 4.4.

\subsection{Previous Work in Offshore Wind Farm Wake Analysis}

The Horns Rev offshore wind farms in the North Sea have been the subject of many offshore wind farm field studies, providing real life wake analyses and a means of validating CFD modelling tools. These early offshore farms are comprised of $2 \mathrm{MW}$ turbines which are small in capacity relative to the latest designs brought to market by current leading manufacturers. Past modelling of the Horns Rev location was done using a modified version of the Park wake model (Katic et al. 1986) which was also implemented in the Wind Atlas Analysis and Application Program (WAsP) [6]. The Park wake model draws upon the Jensen model which uses mass conservation-based wake deficit equations (Jensen 1983) [78]. Katic et al. later modified the Park wake model, suggesting that the square of the total wake deficit should be the summation of the square of all contributing wake deficits. This methodology only accounts for the rotor effects 
and not the 'ground reflecting back wakes'. In a 2015 study done by the Technical University of Denmark's Department of Wind Energy [6], the modified Park wake model was scripted into Matlab in order to derive wake deficits at any given point, enabling the microscale modelling technique to be validated with satellite technology called Synthetic Aperture Radar (SAR) data collected from large offshore wind farms in Northern Europe. Results of the study revealed that microscale models can be useful for understanding inter-farm offshore wake effects. Lateral wake spreads between the SAR imagery and model proved to be very similar. However, the model calculated wake extensions to be $15 \mathrm{~km}$ for the Sheringham shoals wind farm, a much shorter distance than on-site SAR data which revealed wake extension lengths of nearly $30 \mathrm{~km}$. The Sheringham shoals wind farm is an 88 turbine farm with a 317 MW capacity, located off the coast of the UK [79].

SAR was also used by the Risø National Laboratory in Denmark to characterize the wake effects of two Danish wind farms. The Horns Rev is an 80 turbine farm and the Nysted farm consists of 78 turbines. The SAR images revealed wake velocity recovery to be within $2 \%$ of the freestream velocity over a distance of $5-20 \mathrm{~km}$. This variation in wake recovery length was due to the ambient wind speed, atmospheric stability and the number of turbines operating in the 19 SAR images that were collected for the study [80]. The average on site wind speed of the Horns Rev wind farms is $10 \mathrm{~m} / \mathrm{s}$. The Horns Rev farm consists of $2 \mathrm{MW}$ capacity turbines and Nysted turbines are $2.3 \mathrm{MW}$ capacity [81].

Computational fluid dynamics solvers based on the Reynolds Averaged Navier-Stokes (RANS) equations have been used as wake modelling tools beginning nearly two decades ago with the work of researchers such as Duque et al. in 1999 [82]. RANS turbulence modelling techniques belongs to the Statistical Turbulence Model class because the model implements a statistical 
averaging method to derive the equations. Employing these averaging techniques results in a much less computationally expensive simulation compared to a Direct Numerical Simulation [67]. Recent thesis work done by Johnson (2015) demonstrated the reliability of RANS CFD simulations performed using ANSYS-Fluent and ANSYS-CFX for the simulation of wind turbines. This study critically evaluated both codes and found ANSYS-CFX to demonstrate superior performance to ANSYS-Fluent for the computational effort required in the two solvers. Both simulation methods produced results within reasonable agreement to other corresponding data sources in the far wake velocity profiles with the use of the k- $\omega$ shear stress transport model (SST) [48].

\subsection{Rotor Geometry Modelling Techniques in CFD}

Several methods exist to represent the wind turbine rotor in a CFD simulation, all of which vary in their degree of work to build, level of computational expense to resolve, and level of accuracy in the solution. The main methods of representing rotor geometry are: full rotor, actuator line, surface, disk, porous disk, and momentum sinks. Full rotor models are built as a direct representation of a real world wind turbine's geometry, this method is used in the full array simulation and includes the interactions between the turbine blades, tower and nacelle.

Consequentially, this is the most accurate technique of rotor representation. The trade-off for this level of accuracy is that full rotor representation is expensive to simulate. This method requires a sliding mesh to simulate a rotational domain inside a stationary one [48].

Actuator methods, the most commonly used geometry techniques, use a disc, line or surface to represent the blades as a body that exerts a force on the flow field. This body force can be in the shape of a line, disc or surface. Actuator methods require a lower mesh resolution compared to the full rotor case, which in turn reduces the overall computational expense. Momentum sinks 
are the simplest way to represent the turbine blades and would typically be used for far wake studies where the details of turbulent patterns near the blades are not of interest [48]. Porous disk CFD simulations have been found to inaccurately represent the flow regime in the near wake region but produce results closer to experimental comparison cases in the far wake region [83]. Comparison studies between the complex full rotor geometry technique and simpler methods have been conducted. Réthoré et al. researched the differences in results using a full rotor and an actuator disk and actuator line model. Results showed stark differences in computational expense with the full rotor simulation taking much longer to converge [84]. Another study compared the near wake results of a full rotor, actuator disk and actuator line model. In the full rotor case, small structures which have a substantial effect on the development of turbulence were captured in the simulation. Both the actuator disk and actuator line methods were unable to predict these structures. A possible factor of impact on this simulation is that the inlet condition did not have turbulent inflow [85], [48]. Zahle et al. demonstrated good agreement between a single full rotor simulation and experimental data. In this simulation, the rotor's effect on the vortices shed from the tower were successfully captured [86]. Ultimately, using simplification techniques to represent rotor geometry relies on the user to input source terms which are derived from methods such as the lifting line theory. Theories used to compute source terms have a low order of accuracy and neglect the effects of real world factors such as interaction with neighbouring turbines, interactions with the nacelle and tower, yawed flow and turbulence in the atmosphere [87].

To date, most wind turbine wake simulations which use full rotor representation are for a single or two turbine case. Simulations of wind turbines are most often conducted for the purpose of optimization of individual turbine performance. The next progression in CFD wind turbine 
research is to simulate entire wind farms to optimize the performance of a cluster of wind turbines. Most multi-turbine CFD simulations use the actuator line or actuator disk rotor representation. Recent work has been done to model full arrays using full rotor geometry representation, but this work is still highly computationally expensive. In 2013, a University of Wyoming research group undertook the unprecedented task of simulating a wind farm using full rotor geometry representation. The group had access to large computational resources, allowing them to implement a very fine mesh and a very small time step of 0.008 seconds to simulate the intra-farm wake effects of a 48 turbine array. The total time for the full array simulation was estimated to take 8.5 million core hours. An 11,200 core cluster was proposed to carry out the simulation over a 20 day period [87]. Results of this study revealed cumulative wake effects in downstream rows. Turbine power output was reduced from $100 \%$ in the first row to approximately $25 \%$ in successive downstream rows [88].

\subsection{Initial conditions}

In Johnson's review of wake modelling techniques for wind turbines, methodologies for defining the inlet condition are discussed [48]. In the natural environment, wind turbines encounter varying turbulent wind shear [48]. This is referred to as the wind shear profile and can be mathematically defined with the wind power law [89], as shown by Equation 4.1 with $U$ and $h$ as velocity and height from the ground respectively. The variable $U_{r e f}$ is the measured wind speed at a known height, $h_{r e f}$.

$$
\frac{U}{U_{\text {ref }}}=\left(\frac{h}{h_{\text {ref }}}\right)^{\alpha}
$$

The surface of water is smoother than land, and so the wind velocity profile for offshore conditions exhibits a steeper boundary layer than on land. In the Equation 4.1, this factor is 
accounted for by the value of the constant variable, $\alpha$, which is referred to as the wake expansion coefficient.

In CFD two methods exist for incorporating this effect, the precursor and the synthesized inlet. The precursor method, which implements the wind shear profile, is highly unconducive to the meshing process if any changes need to be made to the model geometry or the mesh. Often times, errors in the meshing scheme are not apparent until initializing the simulation. In the initialization of the simulation, negative volumes can be detected which go unnoticed in the set up process. In the precursor method, every time the mesh is altered, the simulation has to be run for an amount of time to obtain the inlet element coordinates in order to initialize the shear inflow parameter.

Alternatively, a synthesized inlet can be created where a turbulent field is created by defining a turbulent length scale for the inlet. These methods of defining the inflow characteristics bring the simulation one step closer to exactly matching the real world case; however, they come at a cost of higher computation expense. Research into the sensitivity of wind turbine wake simulations to turbulent inflow parameters has been done by several researchers referenced by Johnson [48]. Turbulent inflow sensitivity studies using actuator methods have revealed that flow regimes with Reynolds number greater than 1000, show little change from simulating without a turbulent inflow parameter [90-93].

\subsection{Previous Work in Experimental Wake Characterization}

Wind turbine testing procedures were studied by McTavish (2013) using Carleton University's low speed wind tunnel. Many guidelines for conducting wind turbine tests in low speed wind tunnels were highlighted in the work of McTavish which were valuable to this research. McTavish experimented with clusters of wind turbines. In order to fit several turbines 
within the space limitations imposed by a typical wind tunnel, tip speeds with Reynolds numbers on the order of $10^{4}$ were used. Limited work has been done researching the impact of this very low Reynolds numbers on wake expansion. McTavish analyzed low Reynolds number wind turbine experiments which revealed the benefits of using blades tailored to low Reynolds number regimes. Testing revealed that downstream wake expansion became less sensitive to changes above a Reynolds number of 20,000 at the blade tip, but produced narrowing of the downstream wakes which is unlike the wakes of turbines operating in a full scale, commercial array. Vortex pitch was strongly affected by tip speed ratio and lift distribution along the blade in the experimentation. A further objective of the research was to evaluate blockage effects of a closed test section on near wake expansion. Experimental testing with blockage ratios of $6.3 \%$ and $9.9 \%$ produced wake expansions with similar magnitudes to computational results and blockage factors beyond $10 \%$ constricted downstream wake expansion and delayed vortex pairing. Blockage ratio is the ratio of the model's frontal area over the cross-sectional area of the test section [90] . 


\section{Computational Methodology}

The offshore wind farm wake analysis was performed through numerical CFD modelling and was informed by the literature review and the experimental testing described in Chapter 6 . Geometry setup, meshing strategies, CFX setup conditions and convergence criteria of the Eppler simulation which produced congruent results with the experimental hot wire probe results, was implemented in the Sandia offshore simulations, all of which are described in this chapter. The CFD research in this thesis was performed in RANS based, ANSYS CFX.

Three offshore arrays of Sandia 3 MW turbines were simulated. The first case was a two array simulation representative of the potential lake Ontario sites. To reduce computational expense, a cross section of each array was modelled, using the symmetry boundary condition. Array 1 consisted of a $6 \times 4$ configuration and Array 2 consisted of an $8 \times 5$ configuration. For the actual construction of the Trillium wind farm, the total number of turbines in Array 1 will be near 100 . The methodology in choosing a size for the cross section as well as the boundary condition used to account for only simulating a cross-section cut will be described in Section 5.3.2.

Geometric representation of the arrays and the rotor modelling technique implemented is described in Section 5.1. The meshing strategy implemented in the array simulations is discussed in Section 5.2. All set up parameters related to interfaces, boundary conditions, simulation execution controls and time step are described in Section 5.3.

Impact of rotor diameter size on wake recovery length was briefly studied, as described in Section 5.4. The third array simulated was a $7 \times 4$ configuration used to determine the impact of the number of rows on wake recovery length. Influence of increased inlet wind speed was 
another factor analyzed through an additional simulation with the $7 \times 4$ configuration. Both of these additional simulations are discussed in Section 5.5.

\subsection{Model Geometry (Two array simulation)}

As stated in Section 4.2, full rotor modelling is the most accurate technique of rotor representation and uniquely from the other methods, it allows for the other turbine components (i.e. nacelle $\&$ tower) to be included in the simulation. Therefore, full rotor modelling was used in the CFD array models as well as the experimental validation case. As mentioned in Section 4.2, this level of detail causes this method to be labour intensive and computationally expensive.

The overall model geometry of the two array simulation consists of two separate offshore arrays of 3 MW Sandia wind turbines separated by a distance of $7 \mathrm{~km}$ with Main Duck Island near the last row of Array 1. This location is an area surrounding Main Duck Island in Lake Ontario, with the island marked in Figure 5.1 [91]. The approximate locations of proposed build sites are indicated on the map, and the Canada-U.S. border is indicated by the white line. The island and aquatic environment was included in the simulation. Power production in wind farms is reduced to a greater extent in wind farm layouts with axially aligned turbines due to wake effects compared to irregular layouts [92] and so downstream turbines were axially aligned in the simulations of this research to demonstrate the worst case scenario. 


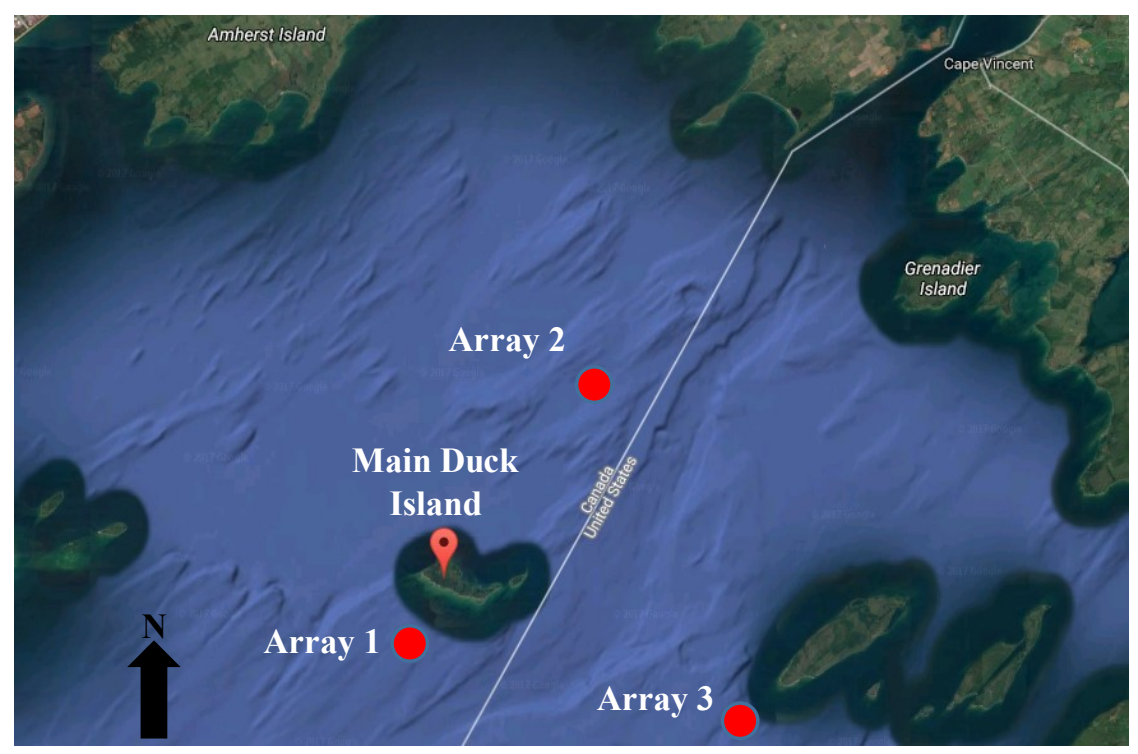

Figure 5.1 Array Locations [91]

Main Duck Island's shores are ideal for offshore wind energy production due to wind conditions and shallow waters. The location of the proposed Array 1 lies $28 \mathrm{~km}$ from the nearest mainland. Array 1 and Array 2 are both proposed build locations for Trillium Wind Power. A development at Array 3 was proposed on the U.S. side of the border by Windstream Energy. Main Duck Island is 407 hectares in size, heavily wooded, and experiences strong winds [93]. Meteorological data indicates that the examined build sites possess wind speeds of at least $8 \mathrm{~m} / \mathrm{s}$ at $100 \mathrm{~m}$ above the water, a typical hub height for a $100 \mathrm{~m}$ diameter wind turbine, and water depths between 5 and 30 meters [94].

A literature review was done to select a publicly-available design similar in capacity, diameter and tip speed ratio to common commercial models implemented in past offshore wind farms. The 3 MW Sandia NASA-Langley design [95] was selected, built in Creo Parametric and imported into ANSYS. This design was selected as early plans of Trillium were to install turbines of the $3 \mathrm{MW}$ size; however, recent technological advancements in the industry have brought about new designs with capacities on the $6 \mathrm{MW}$ and $7 \mathrm{MW}$ scales. In the early design 
stage of the Trillium Wind Power offshore wind project, 3 MW Vesta 90 turbines were planned for the build. If and when approval of offshore wind projects in Lake Ontario happens, some of the arrays will contain the Siemens $6 \mathrm{MW}$ turbines. Unfortunately, due to the novelty of this scale of turbine, data on blade designs similar to the Siemens $6 \mathrm{MW}$ is not publicly available and both simulations consisted of the smaller turbines. Table 5.1 displays a direct comparison of the Vestas-90 and the simulated Sandia NASA-Langley designs.

Table 5.1 Wind Turbine Specifications Comparison [96], [124]

\begin{tabular}{|l|l|l|}
\hline & Sandia hybrid blade (NREL S809/NASA-Langley) & VESTAS 90 \\
\hline Rated Capacity & $3.0 \mathrm{MW}$ & $3.0 \mathrm{MW}$ \\
\hline Blade Length & $50 \mathrm{~m}$ & $44 \mathrm{~m}$ \\
\hline Max Chord & $4.4 \mathrm{~m}$ & $3.5 \mathrm{~m}$ \\
\hline Tip Speed & $70 \mathrm{~m} / \mathrm{s}$ & $67.5 \mathrm{~m} / \mathrm{s}-70.5 \mathrm{~m} / \mathrm{s}$ \\
\hline Rated Rotation Rate & $12.73 \mathrm{rpm}$ & $8.6-18.4 \mathrm{rpm}$ \\
\hline Class & IEC I & IEC I and IEC II \\
\hline
\end{tabular}

Both designs have a rated capacity of $3 \mathrm{MW}$ and have the same range of rotation rates and similar blade lengths. The blade profile specifications used to create the geometry in CREO is included in Table 5.2 [96]. The Sandia NASA-Langley blade consists of ten individual blade elements, each with a unique twist, chord length and thickness. 
Table 5.2 Sandia NASA-Langley Blade Specifications [96]

\begin{tabular}{|l|l|l|l|l|}
\hline Radius Ratio & Radius (m) & Chord Ratio & Chord (m) & Twist (deg) \\
\hline $5 \%$ & 2.6 & $5.2 \%$ & 2.69 & 29.5 \\
\hline $15 \%$ & 7.8 & $7.8 \%$ & 4.03 & 19.5 \\
\hline $25 \%$ & 13.0 & $8.6 \%$ & 4.47 & 13.0 \\
\hline $35 \%$ & 18.2 & $7.6 \%$ & 3.94 & 8.8 \\
\hline $45 \%$ & 23.4 & $6.6 \%$ & 3.45 & 6.2 \\
\hline $55 \%$ & 28.6 & $5.7 \%$ & 2.99 & 4.4 \\
\hline $65 \%$ & 33.8 & $4.9 \%$ & 2.53 & 3.1 \\
\hline $75 \%$ & 39.0 & $4.0 \%$ & 2.09 & 1.9 \\
\hline $85 \%$ & 44.2 & $3.2 \%$ & 1.66 & 0.8 \\
\hline $95 \%$ & 49.4 & $2.4 \%$ & 1.23 & 0.0 \\
\hline
\end{tabular}

For meshing compatibility and to maintain consistency with commercial turbine designs, the top and bottom airfoil profiles were attached with a rounded trailing edge. Figure 5.2 displays the CREO build of the Sandia NASA-Langley design with full-view images and a lateral view of the blade design which reveals the internal blade twist and rounded trailing edge. Each blade is 50 meters in length and the hub diameter is 5 meters. 


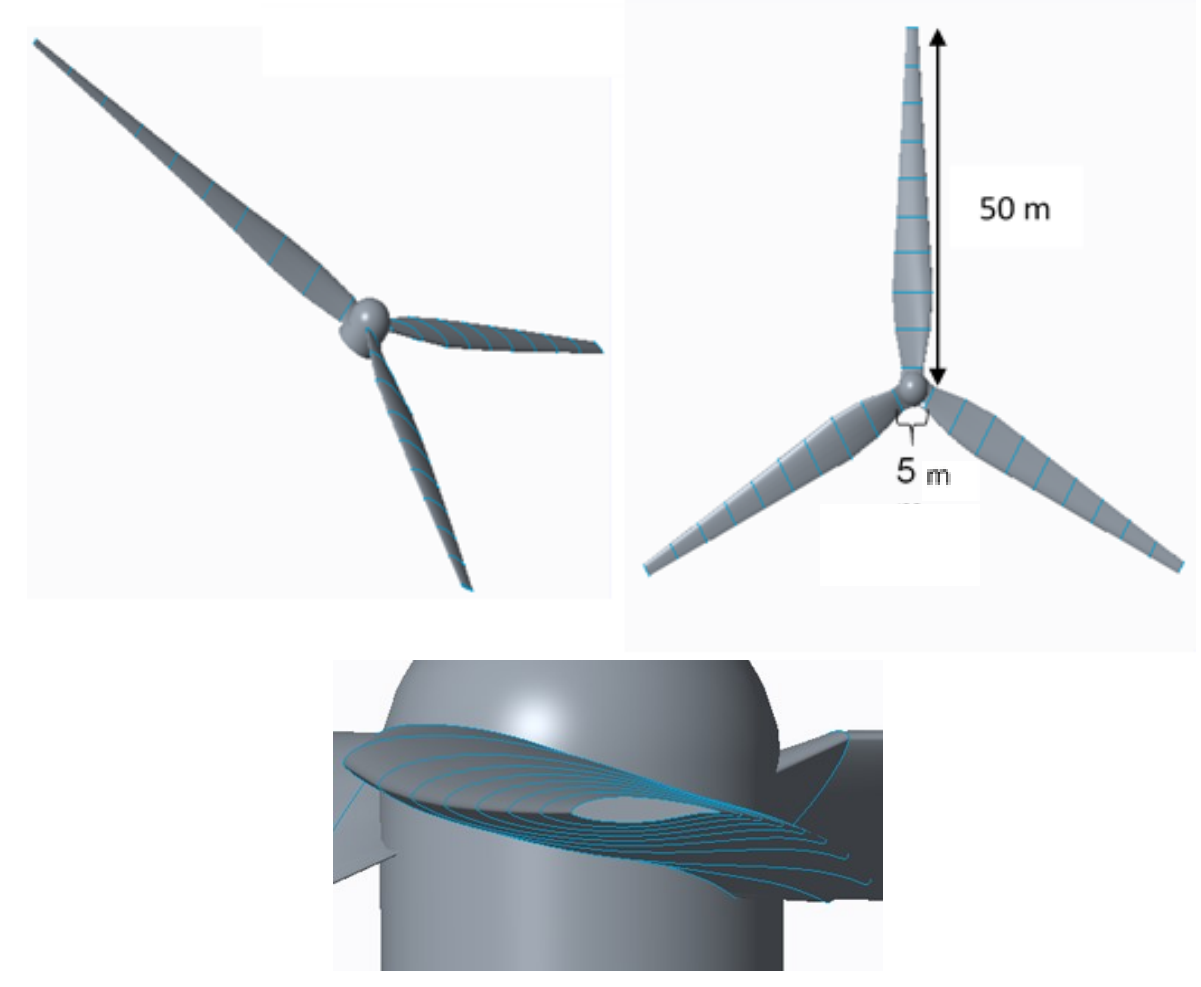

Figure 5.2 Full Rotor Geometry Creo Build

In order to reduce computational expense, the arrays simulated only represent a cross section of the total size of a typical offshore wind farm. All of the cross sections simulated contain the full number of anticipated rows for arrays of this size and a fraction of the columns. The reasoning behind this was to maximize accuracy within the computational constraints as simulating fewer turbines made implementing a finer mesh feasible. Using a greater number of columns than horizontal rows minimizes space requirements of the array as the horizontal spacing requirements are much less than between downstream rows. In addition to optimizing space requirements, maximum power extraction occurs in the first row which is exposed to undisturbed wind. Wind farms are usually constructed in rectilinear layouts [92], the Danish Horns Rev 1 offshore farm being an example. Additionally, when considering wake recovery 
distance, the number of rows has a greater impact than the number of columns in an array. The majority of wake studies focus on axially aligned turbines and analyze the effect of spacing between rows [60]. Array 1 was modelled using 6 rows of 4 turbines and Array 2 consisted of 8 rows of 5 turbines, all placed at standard inter-row spacing distances: 5 rotor diameters in the traverse direction and 10 rotor diameters in the stream wise direction. Both arrays were placed 7 $\mathrm{km}$ apart and built with the highest level of geometric accuracy using full rotor geometry in the simulation. Rotor geometry was directly replicated from a publicly available Sandia design for a $3 \mathrm{MW}$ turbine [96]. Figure 5.3 is a top down view of the entire model.

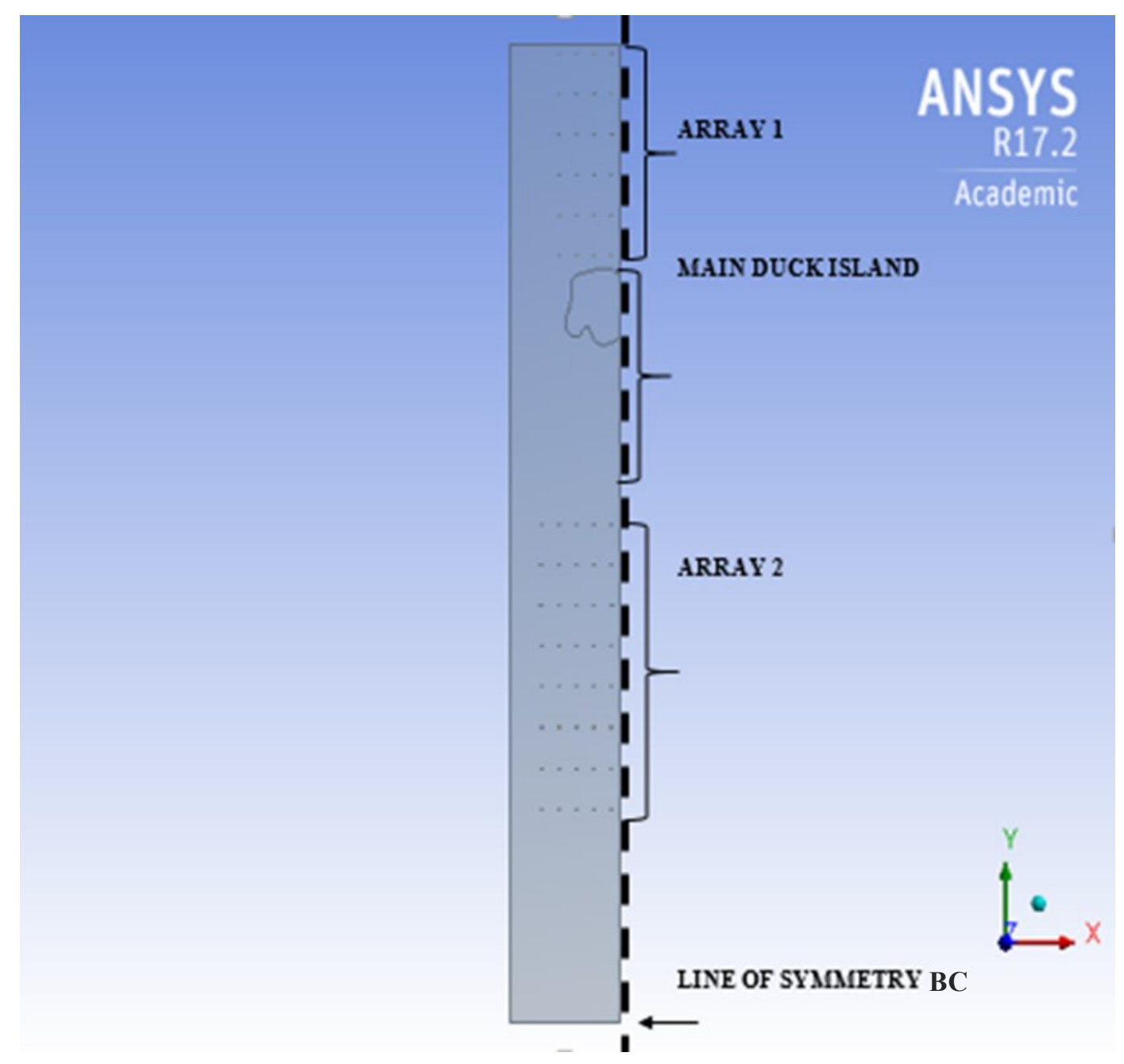

Figure 5.3 Two-Array Simulation Geometry 


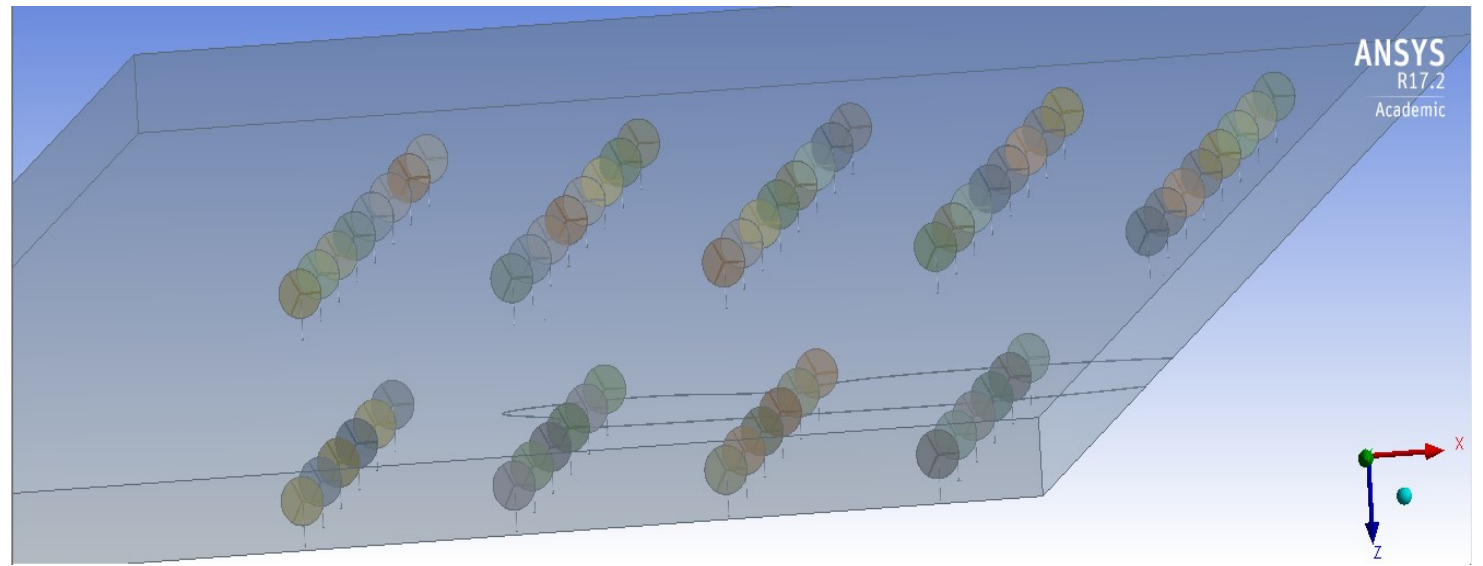

Figure 5.4 Rotational Domains inside the Stationary Environment

As described in Section 3.3.1, modelling requires assumptions that are driven by the objective behind developing the model, computational capacity and the sensitivity of variables in the model's equations. In this study, the purpose is to analyze the overall and cumulative largescale wakes formed from offshore wind farms; therefore, each wind turbine rotor was assumed to be a rigid body. This assumption allowed the wind turbines to be constructed in the simulation as circular disks (rotational domains) with the blade and hub geometry extracted from the rotational domain. Each of the rotational domains was mounted at the hub to a nacelle on top of a $110 \mathrm{~m}$ tower. Rotational domains were encapsulated by a larger stationary domain representative of the offshore environment. An all-encompassing view of the rotational domains inside the stationary domain is pictured in Figure 5.4.

\subsection{Meshing Strategy}

ANSYS CFX is a finite element solver, meaning that conservation of mass and momentum calculations are executed throughout a fluid domain that is divided into elemental pieces. At every node in the domain, calculations are performed. The type of flow problem and the fluid behaviors of interest to the researcher influence the meshing scheme required in the simulation. 


\subsubsection{Grid Size Sensitivity}

A single turbine wake was simulated using three different mesh densities to employ the appropriate grid spacing in the full array simulations. The number of elements in the simulation with the coarsest mesh was approximately half of those used in the finest mesh. The different densities were created by altering the maximum allowable node element spacing in the regions exterior to boundary layer flow. For all mesh comparison simulations, inflation and face sizing tools were used on and around the region of the blades in the same way to have a higher concentration of nodes near the walls. The importance of having a biased accumulation of nodes towards the walls in simulations with turbulent flow will be discussed later on in this chapter along with a description of how this meshing scheme was implemented in CFX. The number of elements in each convergence study is listed in Table 5.3. The velocity in the wake at hub height, across the width of the rotor for distances downstream from the rotor are plotted in Figure 5.5.

Table 5.3 Grid Sizes for Sensitivity Study

\begin{tabular}{|l|l|}
\hline Mesh Type & Number of Elements \\
\hline Coarse & 355978 \\
\hline Medium & 578981 \\
\hline Fine & 724988 \\
\hline
\end{tabular}



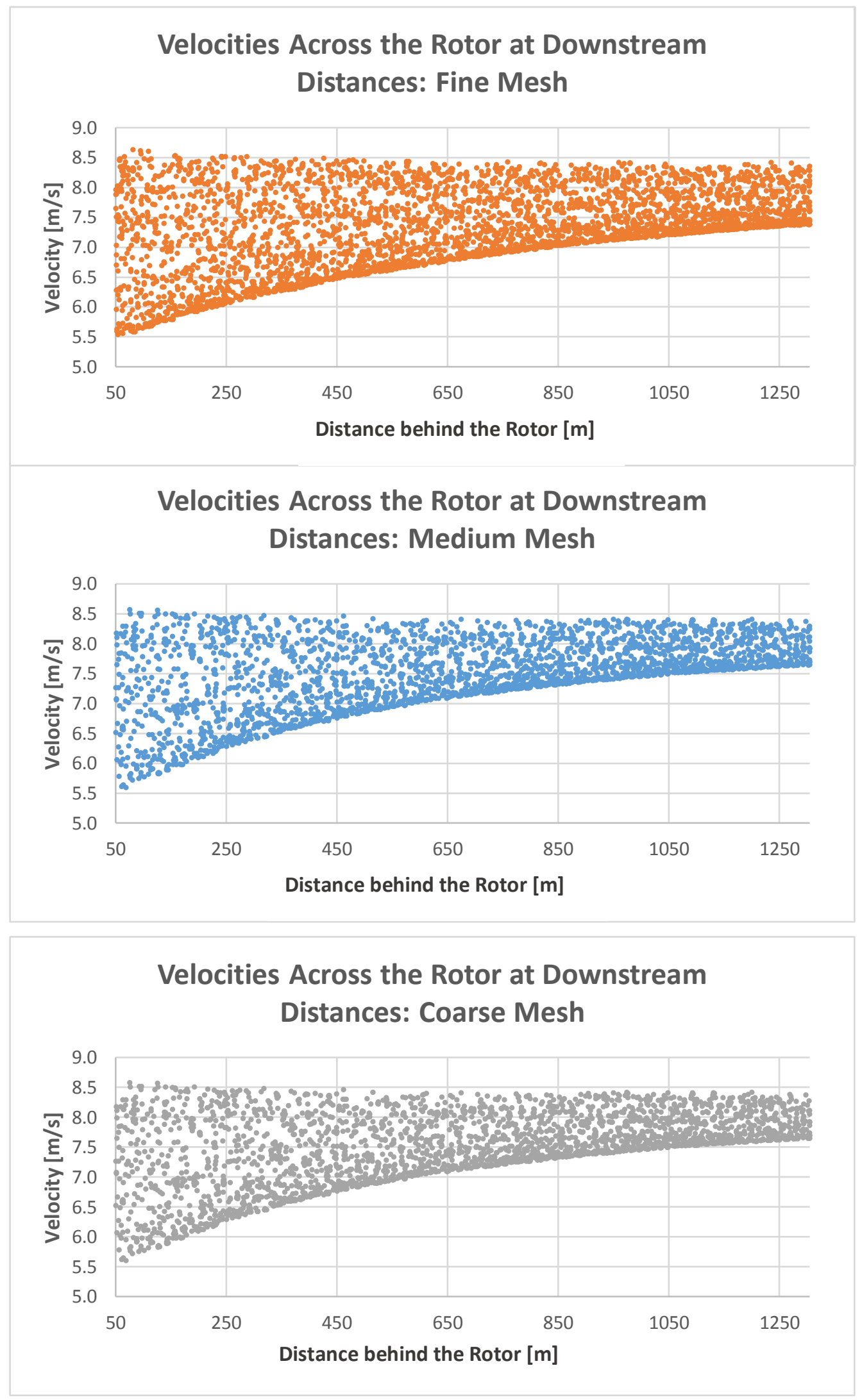

Figure 5.5 Grid Sensitivity Study Velocity Plots 
The percent differences in average downstream velocities between the medium and fine mesh simulations was calculated and plotted in Figure 5.6. $91.1 \%$ of the percent differences calculated were under $1 \%$ and the average of all percent difference calculations was $2.36 \%$. This comparison was done between the "medium" and "fine" mesh simulations. Consequentially, the grid spacing used in the aforementioned "medium" mesh simulation was used in the meshes of the offshore array simulations.

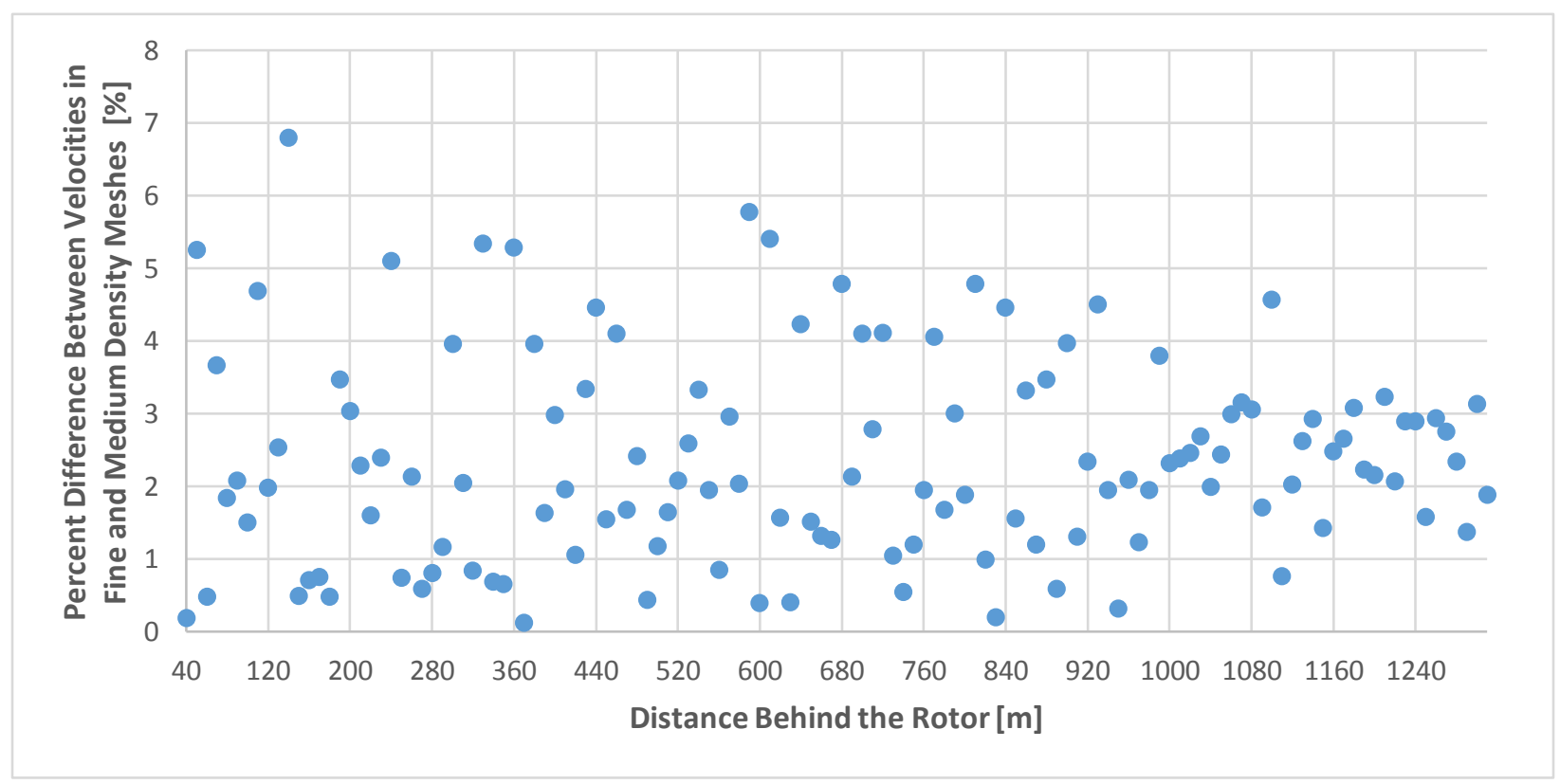

Figure 5.6 Percent Differences of the Grid Sensitivity Study

\subsubsection{Mesh Adaptation for Vortex Shedding}

As a wind turbine extracts energy from the wind, vortex shedding continually occurs as boundary layer separation occurs on the blade surfaces in the high Reynolds number flow regime. This process was described in Section 3.2.1. Boundary layer separation from the wind turbine blades is an integral part of wake development. To improve the precision of boundary layer modelling, a biased accumulation of nodes was created in the boundary layer region using fine layers of rectangular elements, normal to the blade surfaces. Tetrahedral elements made up the mesh throughout the rest of the rotational domains which encapsulate the turbine blades, as 
well as the stationary domain, representative of the offshore environment, nacelles and towers. This type of meshing scheme is well suited for complex geometries. Automatic wall functions employed by the k- $\omega$ SST turbulence model allowed for the near wall mesh to be arbitrarily fine. Sizing of the elements on the blade, tower, hub and nacelle surfaces was limited to $0.4 \mathrm{~m}$. Figure 5.7 is an image of the rotational domain containing the wind turbine blade geometry. Darker shading is representative of the mesh covering the blades and hub of the wind turbine.

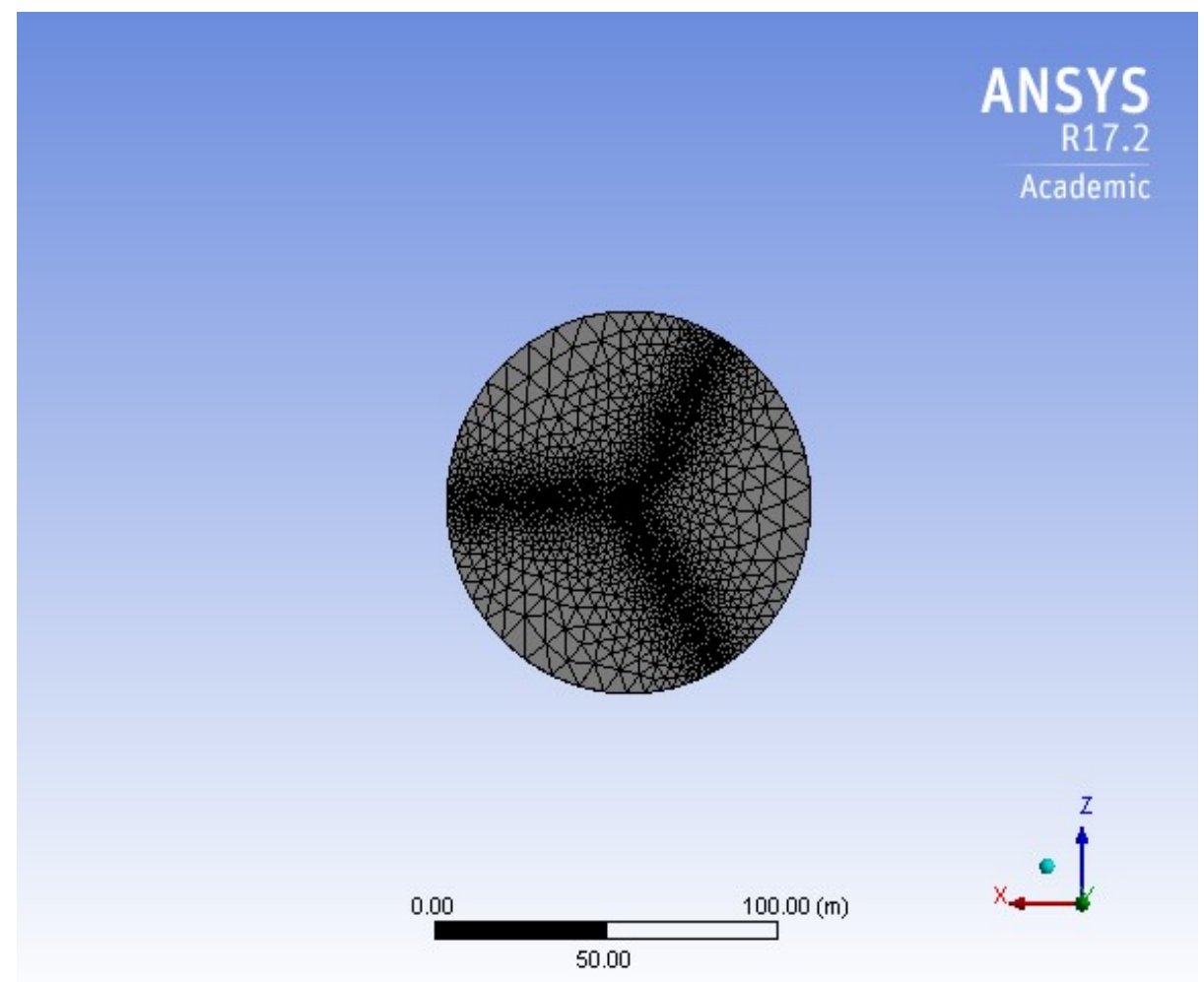

Figure 5.7 Mesh Configuration of a Rotational Domain in the Simulation

The first layer of rectangular prisms surrounding the blade elements were $5 \mathrm{~mm}$ high. Rectangular elements were created using the "inflation" tool in the ANSYS CFX meshing application with a last aspect ratio (Base/Height) of 1.5. In total, 10 layers of rectangular prisms were placed in the boundary layer. Figure 5.8 is a view of the mesh surrounding the airfoil of a blade element. 


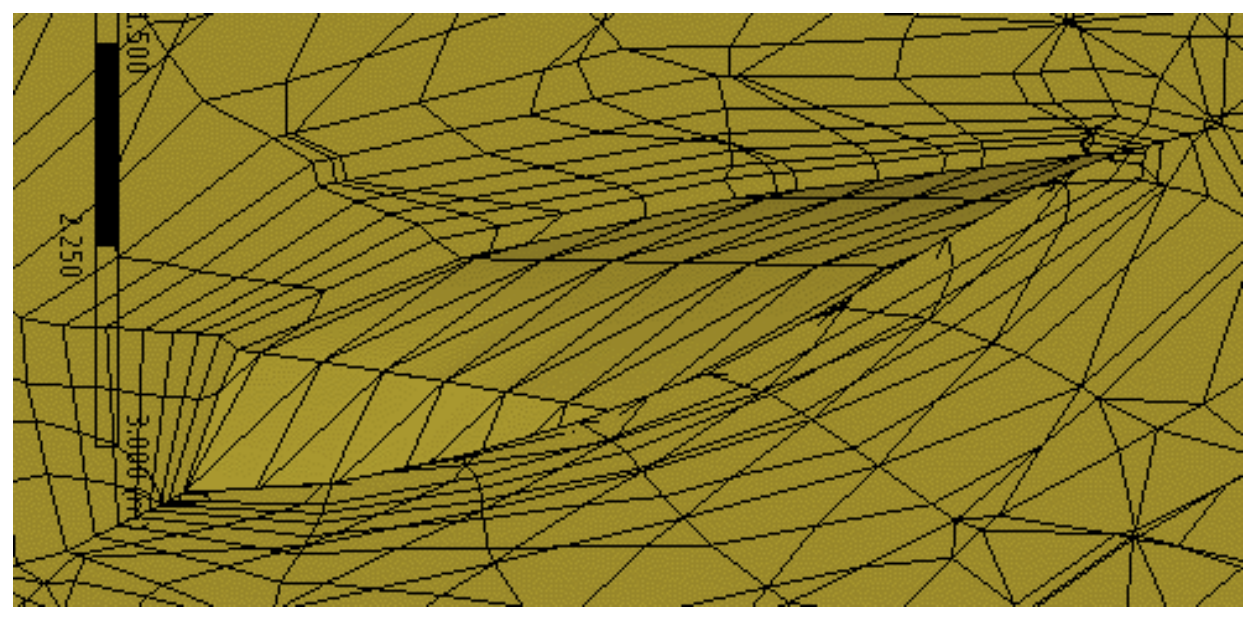

Figure 5.8 Hybridized Mesh Surrounding a Blade Element

The velocity profile of the boundary layer has two distinct regions as fluid transitions from the wall point with the no slip conditions, to the external flow field; the viscous sublayer and the inertia-dominated logarithmic layer, after which the fully- turbulent region lies. This concept was introduced in Section 3.2.1. When performing CFD simulations, the first grid point, normal to the wall surface, must lie within the viscous sublayer region of the boundary layer to sufficiently describe the boundary layer. Using fine rectangular elements, normal to the wall aims to place the nodes nearest to the wall in the viscous sublayer. This distance is determined by the $\mathrm{Y}+$ criterion shown in Equation 6.1, where $\mathrm{Y}$ is the distance from the wall to the nearest node and $v$ is the kinematic viscosity of the fluid and $u_{*}$ is the friction velocity at the nearest wall.

$$
\mathrm{Y}^{+}=\frac{u_{*} \mathrm{Y}}{v}
$$

The degree to which mesh refinement was achieved by creating smaller elements near the blade surface was limited by computational resources and the Courant number, which will be described in Section 5.3.4. Consequentially, the $\mathrm{Y}+$ condition at the high speed region of the blade tips was not achieved directly through the physical mesh element sizing. Often, computational resources do not allow for a mesh refinement level that achieves a $\mathrm{Y}+$ of 1 . 
Additionally, the overall parameter of interest in the research is the large scale cumulative wake effect, and the flow patterns in the boundary layer were not be studied. In cases like this, full resolution of the boundary layer is not required and so the node density near the wall can be greater than what would be needed to satisfy a $\mathrm{Y}+$ condition of 1 [67]. To compensate for the larger Y+ values, wall functions are implemented by ANSYS CFX to approximate portions of, or all of the boundary layer region. In this treatment of the boundary layer through wall functions, an automatic switch from the low-Reynolds method to wall functions occurs when necessary [42]. This automatic near-wall treatment significantly reduces the mesh size constraints in the boundary layer region [67] [97]. Both the k- $\omega$, and k- $\omega$ SST models implement this technique. Wall functions and their role in the two-equation models were described in Section 3.3.2.3. ANSYS theory documentation recommends when using wall functions, to use at least 10 nodes in the boundary layer when using the near wall modelling techniques of the SST model. In this simulation, the SST model was employed and 10 layers were used in the near wall region. Minimum Y+ values must not drop below 11.06 as recommended by ANSYS documentation. The upper range of allowable $\mathrm{Y}+$ conditions is a function of the Reynolds number. In high Reynolds flow regimes (RE 10E9), the solver can handle Y+ values well over 1000 [67].

A fine mesh in the flow field of the farm was also required to capture the vortical structures, but the mesh in this region was also limited by computational resources. Grid spacing throughout the domain was approximately $17 \mathrm{~m}$. This grid spacing was informed by the grid sensitivity analysis. Figure 5.9 shows the entire grid, the total number of grid cells in the simulation was $48,131,055$. 
Complexity of the mesh is increased due to the nature of a stationary environmental domain encapsulating smaller rotational domains. The frames of reference between the rotating domains and the stationary domains are constantly being altered as the blades spin at a defined RPM. Adaptation to account for this behavior is done through the use of a sliding mesh. This technique is applied in the simulation through the use of interfaces described in Section 5.3.1. Through the use of interfaces, the mesh of the rotational domain is continually reconnected in its new position to the stationary domain. 


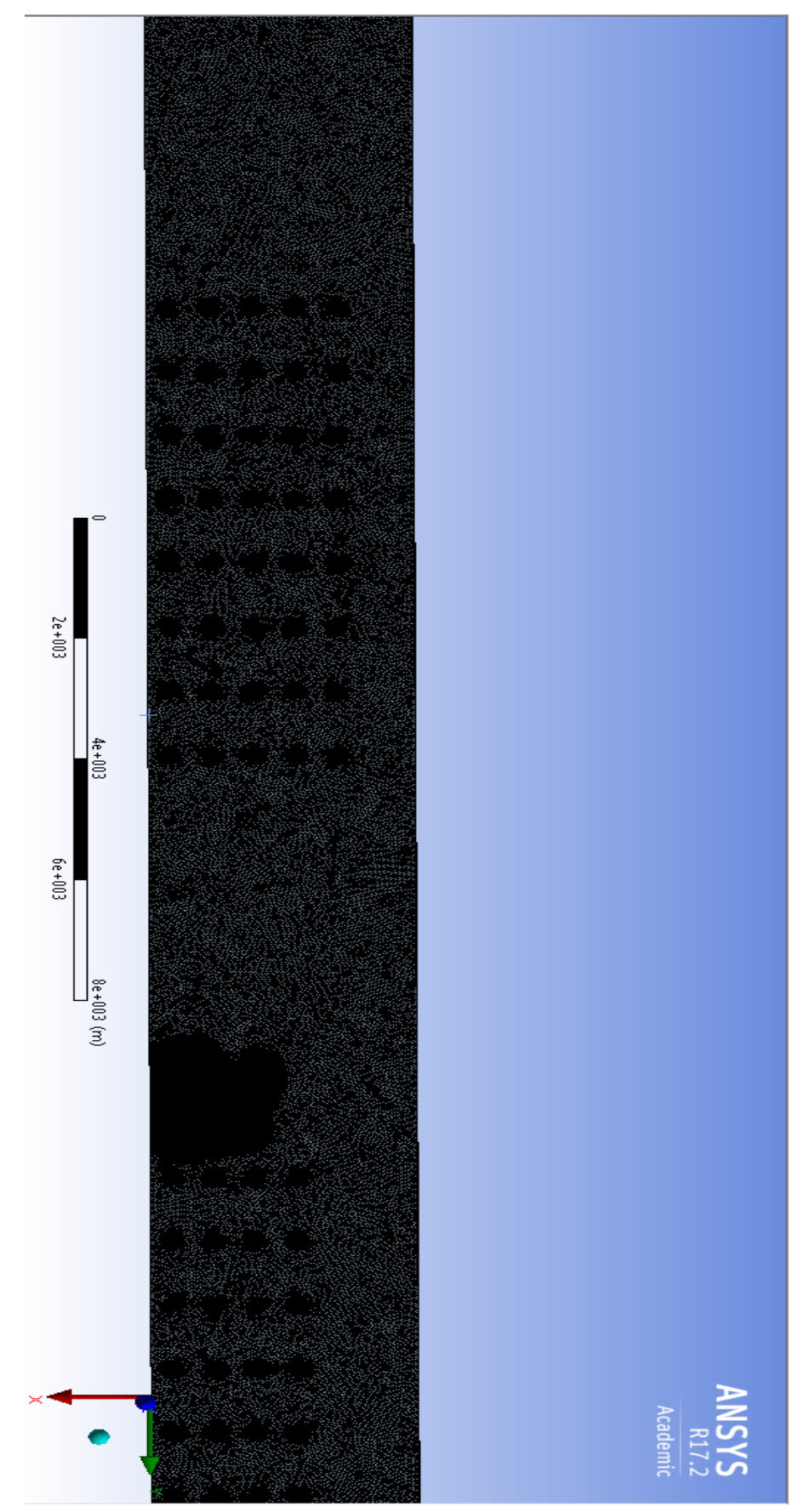

Figure 5.9 Two-Array Simulation Domain Mesh

\subsection{Model Setup in CFX-Pre}

After the mesh has been completed, the next phase of the simulation process is to define the boundary conditions, define the analysis type, and to set expert parameters, memory allocation 
factors, convergence criteria and output control. These set up conditions and what was implemented in the simulations of this work will be described in this section.

\subsubsection{Interfaces}

The complexity level of this simulation is intensified due to the coupling of 64 rotational domains with a large all encompassing stationary domain. In order to "join" the rotational domains with the stationary environmental domain, interfaces were implemented. Interfaces between domains connect meshes which do not match and are used when there are changes in reference frames between domains. The General Grid Interface (GGI) was implemented in the interfaces for the simulations to connect the meshes of the rotating domains to the stationary domain. This setting enables the calculations to proceed throughout the different domains even if the nodes are not perfectly aligned between the domains. In this simulation, the mesh elements are smaller in the rotational domains than in the stationary domain and so the nodes are more densely packed. Fluxes are conserved across the interface. These adjustments made by the interface to conserve all fluxes across the interface between two domains with mismatched meshes and changes in reference frames increases computational expense [98]. The alternative method to GGI would be a more complicated topologically deforming mesh.

In the steady-state simulation, used to obtain initial guess values for the transient simulation, the interfaces were modelled using the "Frozen Rotor" approach. In the "Frozen Rotor" approach, the stationary domain "sees" the rotational domain with the wind turbine rotor at a fixed position as the rotational effects between the domains are not incorporated into the model's equations [99]. Full transient effects of the turning turbine are not modelled, which is why a steady state simulation is insufficient for modelling unsteady flow with vortex shedding that occurs from wind turbine operation. Additionally, losses incurred in the real world scenario from 
the mixing that happens between the stationary and rotating domains is not captured in the steady state simulation [100]. For the transient simulation, the "Transient rotor-stator" interface was implemented. In this frame change option, when particles cross the interface, they continue their motion at the position which corresponds to the position before crossing the interface. The timedependent relative position of the domains is taken into account in this model, unlike in the Frozen Rotor steady state approach [101]. Transient rotor-stator interfaces are ANSYS CFX's most accurate frame change model for simulating flow interactions between rotating and stationary domains. This method is computationally expensive; however, the method predicts the actual transient interaction of flow through the rotor to the stator and simulates the transient relative motion between turbine blades and the fluid in the outside environment on each side of the interface [99].

\subsubsection{Boundary Conditions}

Boundary conditions were applied to all surfaces of the simulation's geometry. All three external sides and top of the stationary domain were defined as openings with a pressure of $0 \mathrm{~Pa}$, simulating being open to the atmosphere. At the cross section cut, the symmetry boundary condition was used in ANSYS, as indicated in Figure 5.10. The symmetry boundary condition assumes that the flow values just adjacent to the domain are the same as the values at the nearest node just inside the domain. The number of rows was made to be a realistic representation of a typical offshore wind farm. Only a quarter of the number of columns was simulated based on the assumption that the number of columns has a negligible effect on wake recovery compared to the effect of the number of rows. In the simulation results, lateral merging of wakes did not occur within both arrays. To further ensure the validity of simulating a cross section of the array, another simulation was performed for a single array containing an extra column. The $6 \times 4$ and 6 
x 5 simulations were compared after 400 iterations and in both cases, the distance from the last row to the end of the wake was near $2300 \mathrm{~m}$.

To simulate the offshore environment, the bottom plane of the stationary domain was defined as wall with a smooth surface. Similarly so, the surface that was the island was defined as a wall with a roughness length of $1 \mathrm{~m}$, the standard roughness length for forested land. All turbine geometry including the blades, nacelles and towers were defined as smooth walls with the noslip condition.

The inlet was defined at a constant $8.78 \mathrm{~m} / \mathrm{s}$. This value was taken from weather data gathered from Lake Ontario at the potential build site. This value was the average flow velocity at hub height of the turbines. Figure 5.10 indicates the locations of the aforementioned boundary conditions in the two array simulation.

Rotational speed of the turbine rotors was set at 12.73 RPMs about their individual axes of rotation manually created in ANSYS CFX. Axes of rotation were created using coordinate frames in CFX-Pre, placing the origins at the center of one turbine in each column. The rotational speed was calculated through Equation 6.2 using an estimated tip speed ratio (TSR), from the tip speed defined in the Sandia NASA-Langley study and the average freestream velocity of the region's area. This freestream velocity was the velocity that was input as the inlet condition.

$$
R P M=\frac{60 * U * T S R}{\pi * D}
$$




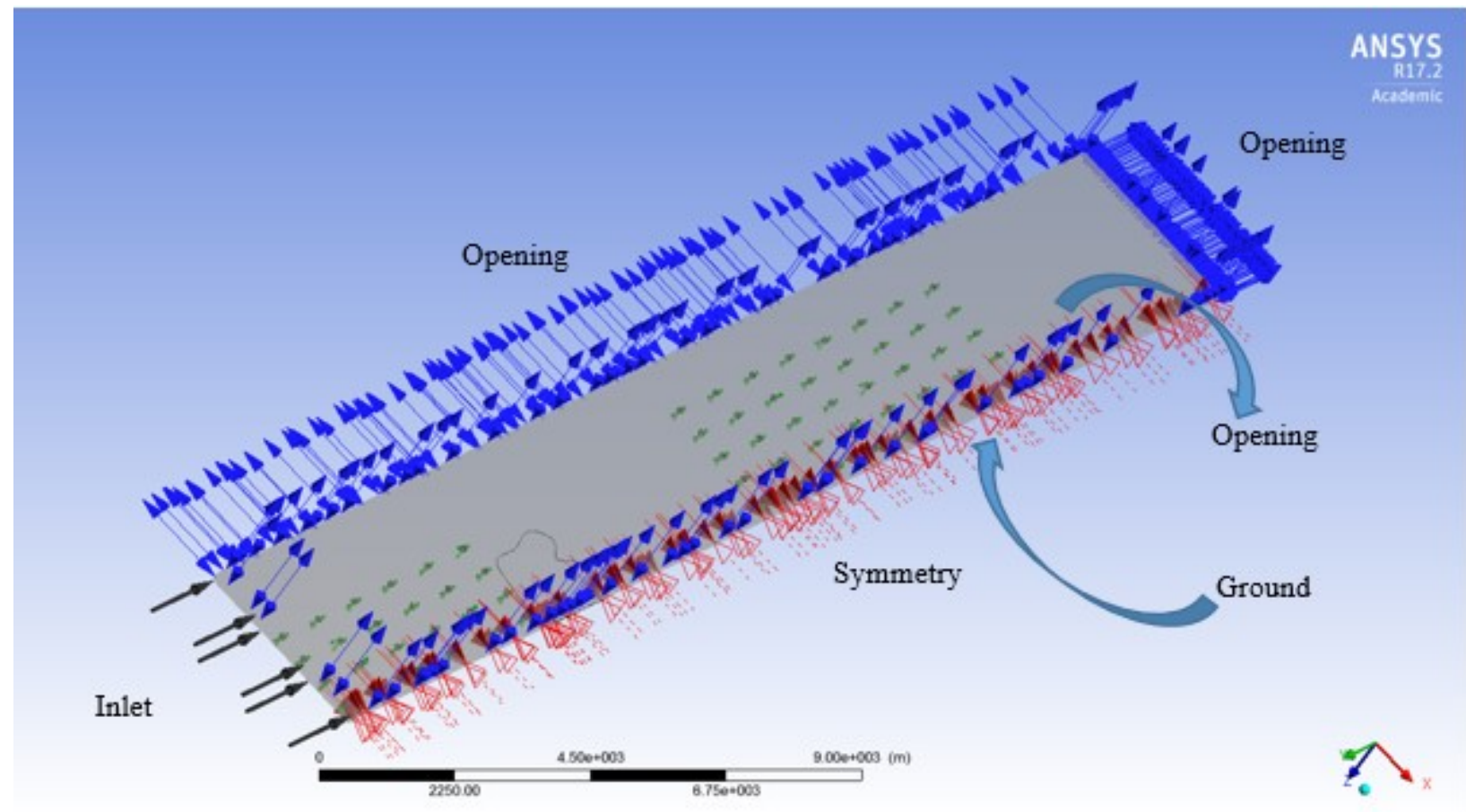

Figure 5.10 Boundary Conditions for the Two Array Simulation

\subsubsection{Simulation Controls}

The analysis type, turbulence model, expert parameters, execution controls and output controls had to be defined. In Section 3.3.3, transient flow problems were discussed and it was stated that the flow regime incurred from rotating wind turbines is a transient process. Consequentially, the simulation was modelled as a transient flow problem. Modelling transient behaviors is computationally expensive; therefore, the steady state simulation was first performed to obtain an initial guess for the transient solver. The results from this simulation were used as an initial guess. Steady state simulation results contain most of the flow features except those which are formed from mixing between the rotational and stationary domains as well as the rotational effects on the stationary domain [100]. These results were input into the solver control in CFXPre to initialize the transient simulation. Transient simulations were performed using the Second Order Backward Euler scheme. The scheme used to calculate advection in the transient 
simulations was high resolution. In the RSM, advection and diffusion terms account for the transport effects of all six Reynolds stresses. Two-equation eddy viscosity models use the advection and diffusion terms to trace the Reynolds stresses [102]. High resolution schemes use a blend factor calculated at each node to be as close to 1 as possible, meaning the formulation is second-order-accurate. This method represents steep spatial gradients with more accuracy than alternative methods [103].

The final run time was decided based upon three criteria: variance in wake recovery length over time, the residual values and imbalance percentages. To determine when the wake recovery length criteria was achieved, intermittent results files were analysed at every 400 time steps. Within the solver's output controls, the simulation was programmed to create a temporary results file every 400 iterations. The wake recovery distance was monitored from each of these output files until the simulation had run long enough for the wake recovery length in the stream-wise direction to remain unchanged between the 400 time step intervals. At this point in time, the first criterion for determining run time was met and the solution had progressed to the steady-state.

The second parameter of run time determination was the residual target. Mass, momentum, turbulence and wall scale equation residual values were monitored at every intermittent results file. Residual values near $10^{-5}$ were considered to be an indicator of convergence. Section 3.3.3 describes residuals and their importance in CFD. Thirdly, imbalance percentages were monitored for the stationary and rotational domains, a concept also discussed in Section 3.3.3. In this simulation, local imbalances were to be well under $0.1 \%$. A summary of the local imbalance values and residuals is presented in Section 7.6. Once the aforementioned criteria were met, the simulation run time was confirmed to be sufficient for determining the wake recovery length of the arrays. The total run time where this occurred was 800 seconds. 
To initiate the simulation, the transient initialization override expert parameter had to be set to "t". This parameter was defined under the physical model tab. Topology estimation factors were set to a value of 1.4 in the expert parameters. This parameter is used to help the solver correctly estimate the memory needed to store the matrix which holds the control volume equations. In large flow problems, the automatic guess for memory requirement made by the solver is often insufficient. The topology estimate factor overwrites the automatic guess and should only be used after an error message recommendation when trying to run a simulation. Sometimes, additional memory estimation overrides are required [104]. In the simulations of this research, the memory allocation factors had to be increased by $60 \%$ in the execution controls.

Because of its aptitude for handling adverse pressure gradients and calculating the amount and onset of flow separation, the k- $\omega$ SST turbulence model was used to run the ANSYS simulation. The application of the wall functions used by the k- $\omega$ SST model reduced computational expense and provided a more accurate way to simulate the boundary layer compared to the k- $\varepsilon$ model, while also allowing for the implementation of an arbitrarily fine mesh. The k- $\omega$ SST model was described and contrasted with other turbulence model options in Section 3.3.2.3. Lastly, the size of the array simulations required significant computational resources and so all simulations were computed using a Carleton computing resource, clusterq, with 32 cores and 128 GB ram.

Command lines are used to run jobs on the clusterq through a secure shell connection. Using the cluster, the two-array simulation converged in approximately 26,880 core hours.

\subsubsection{Time step}

The final simulation was run using transient models which requires a time step and run time to be defined in CFX Pre. In CFD, the Courant number is a constraint used to determine an appropriate time step. The Courant can impact residual values in the model as well as the net flux 
through the domain. This parameter is essentially a measure of how many grid cells fluid is transferred over during a time step. If the courant number is greater than 1 , a single fluid particle would travel through more than one cell each time step. This parameter is used as a criteria for convergence in numerical solvers, specifically in explicit ones where the equations are discretized in such a way that there is only one unknown variable to be solved for in an equation using known variables from the previous time step [105]. The Courant-Friedrichs-Lewy (CFL) condition is where the Courant number, solved for using Equation 6.3, equals approximately 1 [106]. Density based explicit solvers are limited by this condition [107].

$$
C=\frac{u \Delta t}{\Delta x}, \text { CFL condition }: \mathrm{C} \approx 1
$$

In flow regimes with rotor components of large diameters, high speeds are present at the blade tips, and decreasing the Courant number becomes a challenge as shown in Equation 6.3. In the flow simulation for this research, the fluid velocity climbs to ranges of $70 \mathrm{~m} / \mathrm{s}$ at the blade tips, presenting a challenge in achieving the CFL condition. Equation 6.3 illustrates that decreasing the time step $(\Delta t)$ in the solver will reduce the Courant number. Unfortunately, small time steps increase the simulation convergence time. Increasing grid element sizes is an additional way to decrease the Courant number; however, the nature of the flow around the blade surfaces is key to wake formation, requiring small elemental size in this region of high flow velocity. Achieving a reasonable $\mathrm{Y}^{+}$criteria also requires smaller element sizes in regions of high flow velocity.

The CFL condition was achieved in the array simulations in the stationary domain but not in the rotational. Due to aforementioned high velocities and small element sizes around the blades, simulations with Courant numbers below 1 in all rotational domains would become extremely computationally expensive. The impact of making this compromise of accepting a higher Courant number in the simulation has been explored. In this simulation case, the flow problems 
at hand involve irregular, turbulent motion. Complexities in this flow regime are characterized by non-linear, equations. Implicit solving schemes are used in this ANSYS CFX simulation to derive a solution at each time step through a system of non-linear algebraic equations for the whole mesh. In this scheme, the variables depend on parameters at the current time step, unlike in explicit techniques where the unknowns are all dependent variables which rely on the solution calculated in the previous time step. At each time step, the solver uses iterative techniques to obtain a solution rather than directly computing the dependent variables in terms of known quantities. Each time step is more computationally expensive using the implicit technique, as more space is needed in the matrix of control volume equations to solve. Implicit formulation is advantageous because the system of equations are highly stable and so the solution does not diverge for certain parameters like time step size [108]. This solving scheme can handle larger time steps and therefore larger Courant numbers. In the RANS-based simulations for this research, the Courant number does not need to be small to maintain stability. In explicit methods, a small enough Courant number must be employed for stability. When using Large Eddy Simulation (LES), a small courant number must be chosen to correctly simulate the intricacies in the transient flow. In the setup of ANSYS, for all runs in this work, the automatic time step initialization option was implemented with the Second Order Backward Euler scheme. In this methodology, the Courant number is used to determine if the solution from the previous time step is used to resolve the equations at the current time step, or if the solutions from previous time steps should be extrapolated to the new time step. By default, extrapolation is used if the Courant number is less than 5 and the previous time step is used if the Courant number is above 10 [109]. 
Although the implicit solver used is not extremely limited by the Courant number and time step size, the impact that this variable has on the solution is important to consider. For the full rotor simulation of the 2 array scenario, the average Courant number in the entire fluid domain was 2.95. The maximum Courant number in the rotating domains was 57.5 , in the high fluid velocity region of the blade tips. A courant number under 1 was maintained throughout the stationary domain.

A sensitivity analysis was performed to determine the effects of reducing time step on simulation results. Several simulations for a single $3 \mathrm{MW}$ turbine were performed several times while varying the time step in order to determine the effect of Courant number on the convergence and the sensitivity of simulation results to the alteration. In an NREL study, the sensitivity of wind turbine CFD actuator simulations to time step was analyzed. The results of this part of the study suggested that the time-step did not cause a significant difference in the simulation results [110].The impact of Courant number was analyzed on the full rotor geometry of this research through convergence studies, altering the time step and grid densities which are described in Section 5.3.4.1.

\subsubsection{Time Step Sensitivity Study}

Three separate simulations of a single Sandia 3 MW turbine were performed using a different time step in each simulation. All other parameters of the simulation were the same in each simulation. The first used a small time step of $\Delta t=0.1 \mathrm{sec}$. This time step was doubled in the second simulation and quadrupled in the third. Because the differences in wake recovery between these three results were negligible, the largest time step of the aforementioned simulations, $0.4 \mathrm{sec}$, was used in the full-scale simulation to optimize computational expense. Using this time step, the solver iterated at every 30 degrees of rotation. Figure 5.11 is a plot of 
the velocity values at downstream distances behind the turbine rotor at hub height, across the width of the rotor for the all three time step comparison simulations. Differences between the simulations are most pronounced in the near wake zone, 2 -4 rotor diameters downstream of the turbine. At $40 \mathrm{~m}-60 \mathrm{~m}$ downstream from the rotor, velocities across the rotor range from $6.17 \mathrm{~m} / \mathrm{s}$ to $7.67 \mathrm{~m} / \mathrm{s}$ in the $\Delta \mathrm{t}=0.1 \mathrm{sec}$ and $5.75 \mathrm{~m} / \mathrm{s}$ to $6.92 \mathrm{~m} / \mathrm{s}$ in the $\Delta \mathrm{t}=0.4 \mathrm{sec}$ simulation. At $600 \mathrm{~m}$ downstream from the rotor, these differences become notably smaller with the average velocities across the rotor for $\Delta \mathrm{t}=0.1 \mathrm{sec}$ and $\Delta \mathrm{t}=0.4 \mathrm{sec}$ simulations being $6.16 \mathrm{~m} / \mathrm{s}$ and $6.13 \mathrm{~m} / \mathrm{s}$, respectively. 


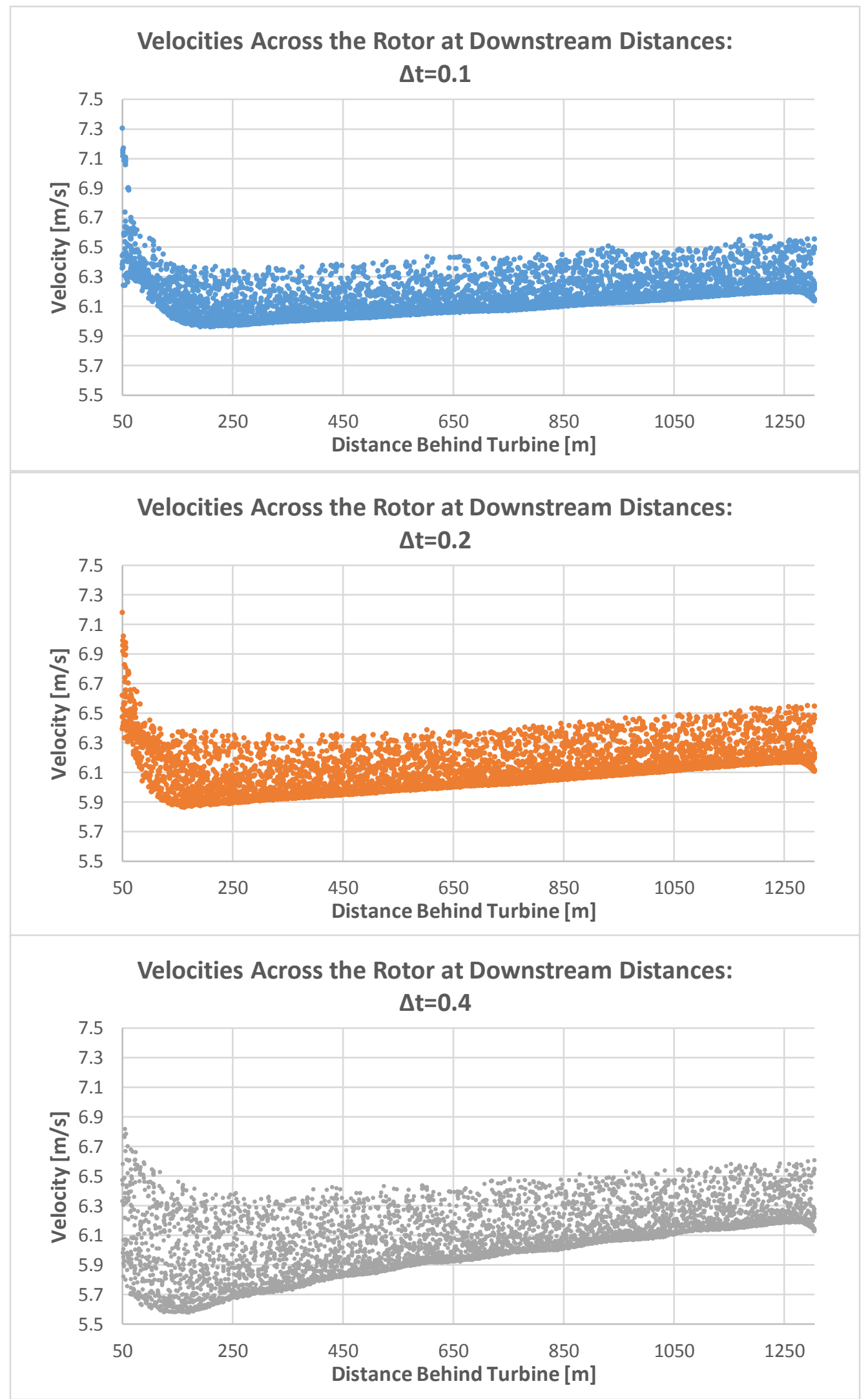

Figure 5.11 Velocity Comparison Plots from Time Step Study 
The differences in velocity between the time step comparison studies become less, farther downstream from turbine. From $1250 \mathrm{~m}$ to $1300 \mathrm{~m}$ behind the rotor, the cumulative average velocity of all points across the rotor were $6.28 \mathrm{~m} / \mathrm{s}$ for both the $\Delta \mathrm{t}=0.1 \mathrm{sec}$ and $\mathrm{sec} \Delta \mathrm{t}=0.4 \mathrm{sec}$ simulations respectively. The node to node percent differences for velocity behind the rotor between the $\Delta \mathrm{t}=0.1 \mathrm{sec}$ and $\Delta \mathrm{t}=0.4 \mathrm{sec}$ simulations are plotted in Figure 5.12. At $600 \mathrm{~m}$ and beyond, the node to node percent differences between the $\Delta t=0.1 \sec$ and $\sec \Delta t=0.4 \sec$ simulations are all below $2.4 \%$ and at $1000 \mathrm{~m}$ behind the rotor, $98.6 \%$ of the percent differences are below $1 \%$.

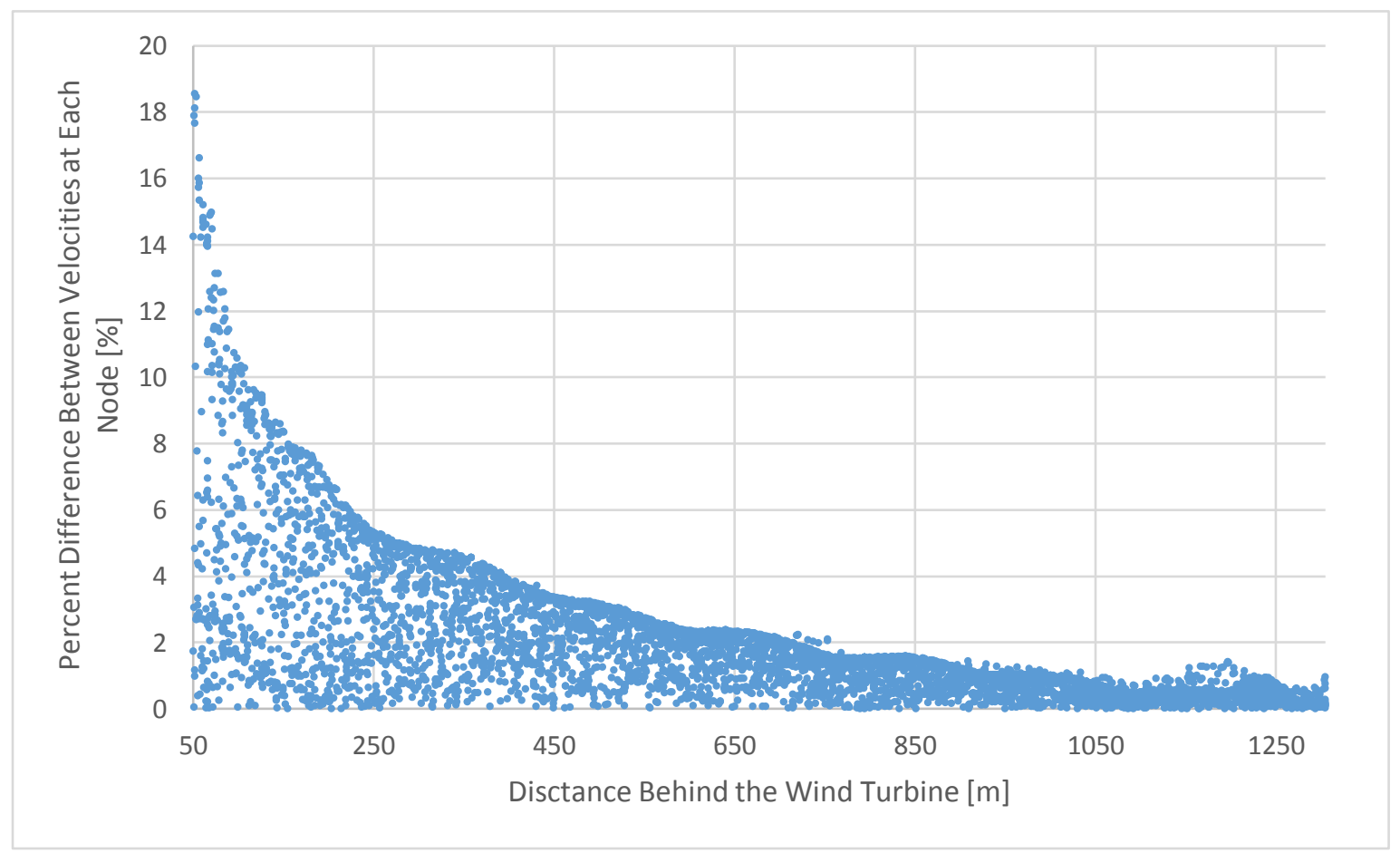

Figure 5.12 Percent Difference between Nodes of the Largest and Smallest Time Step

\subsection{Rotor Size Comparison Simulation}

A brief study was done on the effect of rotor diameter size on the wake recovery distance in the stream-wise direction. Two simulations of a single turbine were performed for a physical time of 800 seconds; one for a single Sandia NASA - Langley 3 MW size design with a diameter of $105 \mathrm{~m}$ and one with the aforementioned geometry scaled up to a diameter of $154 \mathrm{~m}$. This size of 
the larger diameter case was the same as the Siemens $6 \mathrm{MW}$ model. All of the set up parameters described in this chapter for the array simulations were implemented in this model in the same way except for the rotational speed of the turbine. The adjusted rotational speed was based on the Siemens $6 \mathrm{MW}$ offshore wind turbine technical specifications [111]. The same inlet wind speed was used in both simulations and the estimated RPM in this regime was 6 RPM for the large rotor. The rotational speed of the smaller rotor was 12.73 RPM as in the other simulations. This was calculated by considering the rotational speed range for the Siemens $6 \mathrm{MW}$ turbine (5 to 11 RPM) and the cut-in and cut-out speeds which are $3-5 \mathrm{~m} / \mathrm{s}$ and $25 \mathrm{~m} / \mathrm{s}$ respectively.

\subsection{Additional Simulations}

An additional study was performed to determine the relationship between numbers of streamwise rows of turbines in an offshore farm. In the large two array simulation described in Sections 5.1-5.3, a 6 x 4 array and an 8 x 5 array were simulated. In the additional row comparison study, a $7 \times 4$ array was simulated under the same conditions as in the two array model. The meshing strategy, solver controls, run time, time step and convergence criteria implemented into the 7 x 4 array model were all consistent with the two array model. Results of this simulation are presented in Section 7.2.

As described in Section 5.3.2, symmetry was implemented in to the simulation in order to only represent a cross section of a full array. The number of rows was kept consistent to that of a typical commercial wind farm, but only a fraction of the columns were simulated. Array configuration was set up this way due to the assumption that the number of rows has a greater impact on wake recovery distance than the number of columns. In order to verify this assumption and test the simulations' sensitivity to the number of columns, a 6 x 5 array was simulated. At 
400 time steps, the wake velocity contour plots of the $6 \times 4$ and $6 \times 5$ arrays were compared and produced a solution with the same wake recovery length.

An additional study was performed to determine the sensitivity of the simulation to the inlet condition, the effect of wind speed on wake recovery length was looked at. In this study the exact same file used to simulate the aforementioned $7 \times 4$ array was used. Wind speed was the only variable altered and was increased by $10 \%$. Results of this study are presented in Section 7.3 . 


\section{Experimental Methodology}

Experimental testing was used to validate the simulation techniques used in the research, validating the numerical simulations beyond the CFD convergence study, described in Section 7.6. Experimental validation was done through wind tunnel testing on a single $25 \mathrm{~cm}$ diameter wind turbine with a blade designed by McTavish (2013) which used the Eppler E387 airfoil [90]. The $1.68 \mathrm{~m} \times 1.12 \mathrm{~m}$ atmospheric boundary layer wind tunnel at Carleton University was used for the experiments. As described in Section 4.4, experimental wind turbine testing with blade geometries tailored to low Reynolds number flow regimes are better suited for wake analysis. This is why a different blade design was used for the CFD validation through experimentation rather than scaling down the 3 MW SANDIA design to a size that would be testable in the wind tunnel.

The Experimental apparatus was replicated in ANSYS CFX and simulated under the same conditions as in the wind tunnel experiment. The geometry of the wind turbine in the ANSYS simulation was modelled using the full rotor technique. The wake was characterized using a hot wire anemometer system and evaluation of the results revealed acceptable confirmation of the CFD methodology with a cumulative difference of $9.53 \%$ between the numerical and experimental results. The same methodology used in the CFD simulation for comparison to the experiment was employed in the large scale simulation of offshore arrays. The results of this study are presented in more detail in Section 7.5.

\subsection{Experimental Components}

The apparatus for data collection consisted of a hot wire sensor probe with a hot wire probe support attached to a traversing system using T-slot bars, an anemometer, a data acquisition system (DAQ) and a computer with LabView to read the data in LabView and control the sensor 
and moving of the probe in the flow field. The traverse system design and LabView code were developed in the McTavish 2013 study. The hot wire probe was purchased from Dantec Dynamics and was $5 \mu \mathrm{m}$ in diameter, with a $1.25 \mathrm{~mm}$ long plated tungsten wire sensor. The anemometer was a single channel constant temperature anemometer (CTA) from A.A. Labs systems. Figure 6.1 is a visual flowchart of the data collection process.

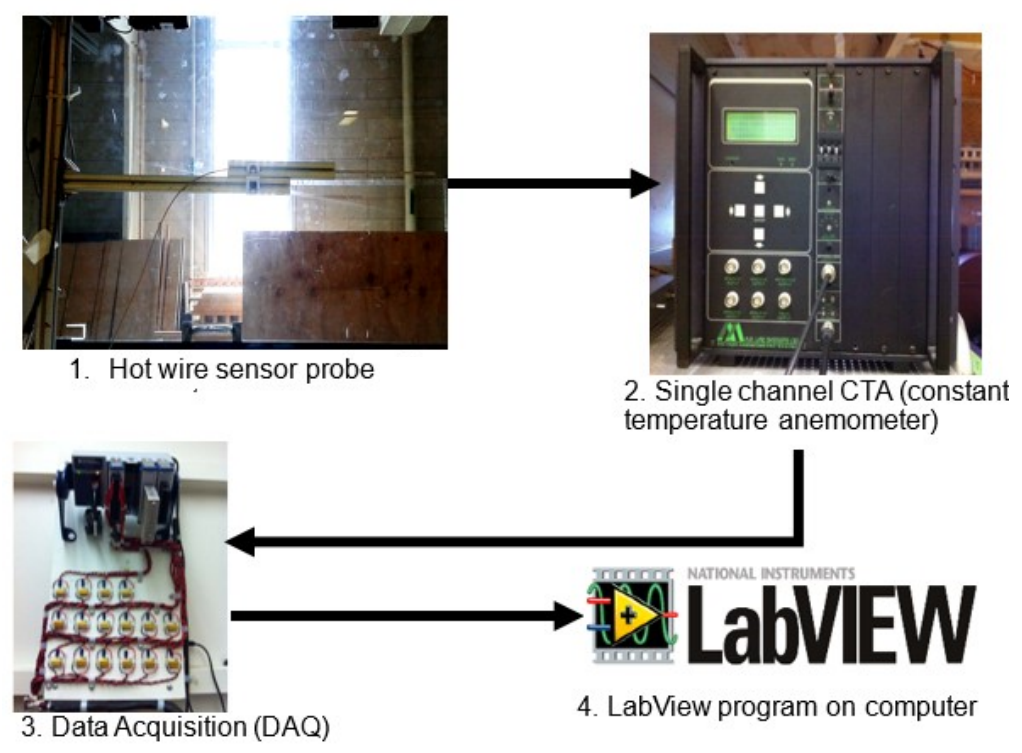

Figure 6.1 Experimental Procedure Flowchart

The test wind turbine is pictured in Figure 6.2. For testing, the model wind turbine was mounted in the centre of the wind tunnel test section, upstream from the traversing system which supported the hot wire. 3D printing the blades, done by McTavish (2013), was executed with a high resolution printer. 


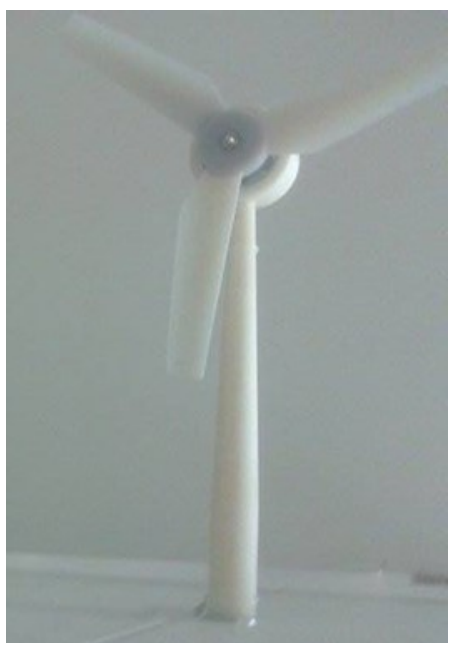

Figure 6.2 Model Wind Turbine Used in Experimental Testing

A diagram of the hot wire probe is pictured in Figure 6.3. The wire is heated to a constant temperature which changes in response to fluid velocity changes in the wake of the turbine. The voltage required to keep the wire heated at the constant temperature is read out from the circuit and this voltage difference corresponds to a specific wind speed. This wind speed can be determined from the calibration curve.

\section{Constant temperature wire sensor}

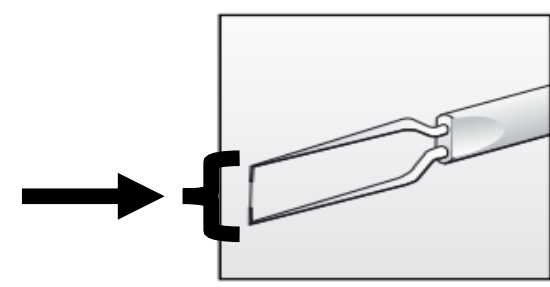

Figure 6.3 Hot Wire Probe

The channel was set up following manufacturer guidelines before experimentation. The CTA contains a signal conditioning unit used to amplify, offset, and filter the signal. The gain and offset were adjusted to match the range of expected flow conditions to the voltage output range of the anemometer. The sampling rate of the anemometer was kept consistent with the experimental work done by McTavish [90]. Data was passed through a low pass filter at a 
frequency of $1.4 \mathrm{kHz}$ and sampled at $2.8 \mathrm{kHz}$. The Data Acquisition system (DAQ) receives the signal from the CTA and outputs 65,536 discrete data points for each experimental run. The DAQ sends information to the PC where the data can be viewed using a program called LabView. Additionally, the LabView- DAQ system acts as a control to initialize data collection. Processing of the data was performed in Matlab where the mean values of the voltage readouts for the experimental runs were found. These voltages were used to determine the velocity using the calibration curve obtained before and throughout the experiment.

\subsection{Experimental Procedure}

Calibration was done to determine the flow velocity values that corresponded to the sensor's voltage outputs. The flow velocity at the sensor's location was measured using an inclined manometer with a pitot tube. Before generating the calibration curve, the resistance of the cable was zeroed out and the resistance of the probe was determined and reset with an over-heat ratio factored in. After calibration, a single wind turbine was placed in the centre of the wind tunnel test section. Flow velocity in the tunnel was set to a constant $21 \mathrm{~km} / \mathrm{h}$ and measurements were then taken with the hot wire probe in the wake of the wind turbine, $17 \mathrm{~cm}$ downstream from the turbine hub. Hot wire probing was started at a location near the tunnel wall, in the freestream. The probe was moved with the motor-powered traversing system controlled through LabView. Measurements were taken at $5 \mathrm{~cm}$ lateral increments for each row of measurements behind the turbine. Six different downstream rows of data points were collected, spaced at $6 \mathrm{~cm}$ increments. All measurements were recorded at hub height of the turbine. Maintaining the lab at a constant temperature was difficult and consequentially, unobstructed flow speed measurements had to be taken after every row of data collection to formulate the calibration curve which best represented the voltage readings of each individual row. 


\section{Results}

The purpose of this research was to study the cumulative wake effect of clusters of offshore wind turbines. To do this, two CFD simulations were performed in ANSYS CFX. The first simulation was a two-cluster simulation based on potential build sites in Lake Ontario proposed by Trillium Wind Power. The upstream cluster of wind turbines in this simulation was a 6 x 4 array, and the downstream cluster was an $8 \times 5$ array. To show the effect of number of rows on wake recovery distance, a second CFD simulation was completed with a single $7 \times 4$ array using the same boundary conditions as the first simulation. Results of the aforementioned CFD simulations are presented in Section 7.1 and 7.2. Sensitivity to inlet wind speed was also analyzed as presented in Section 7.3. For additional information, a brief study on the effect of rotor geometry on wake recovery was done and the results are shown in Section 7.4.

Wind tunnel testing was done to validate the methodology used to build the aforementioned offshore array simulations. Hot wire anemometry was used to characterize the wake behind a small scale wind turbine in the wind tunnel. This experimental test was replicated in ANSYS CFX and simulated under the same boundary conditions as the experimental run. Experimental results are presented in Section 7.5, with a comparison to the CFD results. To verify the offshore array simulations, time step and grid size convergence studies were performed to inform the time step and grid densities implemented in all the simulations of this research. Lastly, a convergence study is presented for the two array simulation in Section 7.6.

\subsection{Two Array Simulation (6 $x 4$ and $8 \times 5)$}

Simulation results show that wind velocity would be approximately $89 \%$ recovered compared to that of the freestream velocity at $6.8 \mathrm{~km}$ behind the last row of the $6 \times 4$ array. At this point in the flow field, the average wind speed is $7.83 \mathrm{~m} / \mathrm{s}$ which is within $1 \mathrm{~m} / \mathrm{s}$ of the freestream 
velocity at the domain inlet. Figure 7.1 is a plot of the averaged resultant velocities over the rotor diameter for increasing distances behind the $6 \times 4$ array. The downstream proposed array location is $7 \mathrm{~km}$ away from the $6 \times 4$ array and so the exact point of $100 \%$ recovery to freestream levels could not be determined for the $6 \times 4$ array.

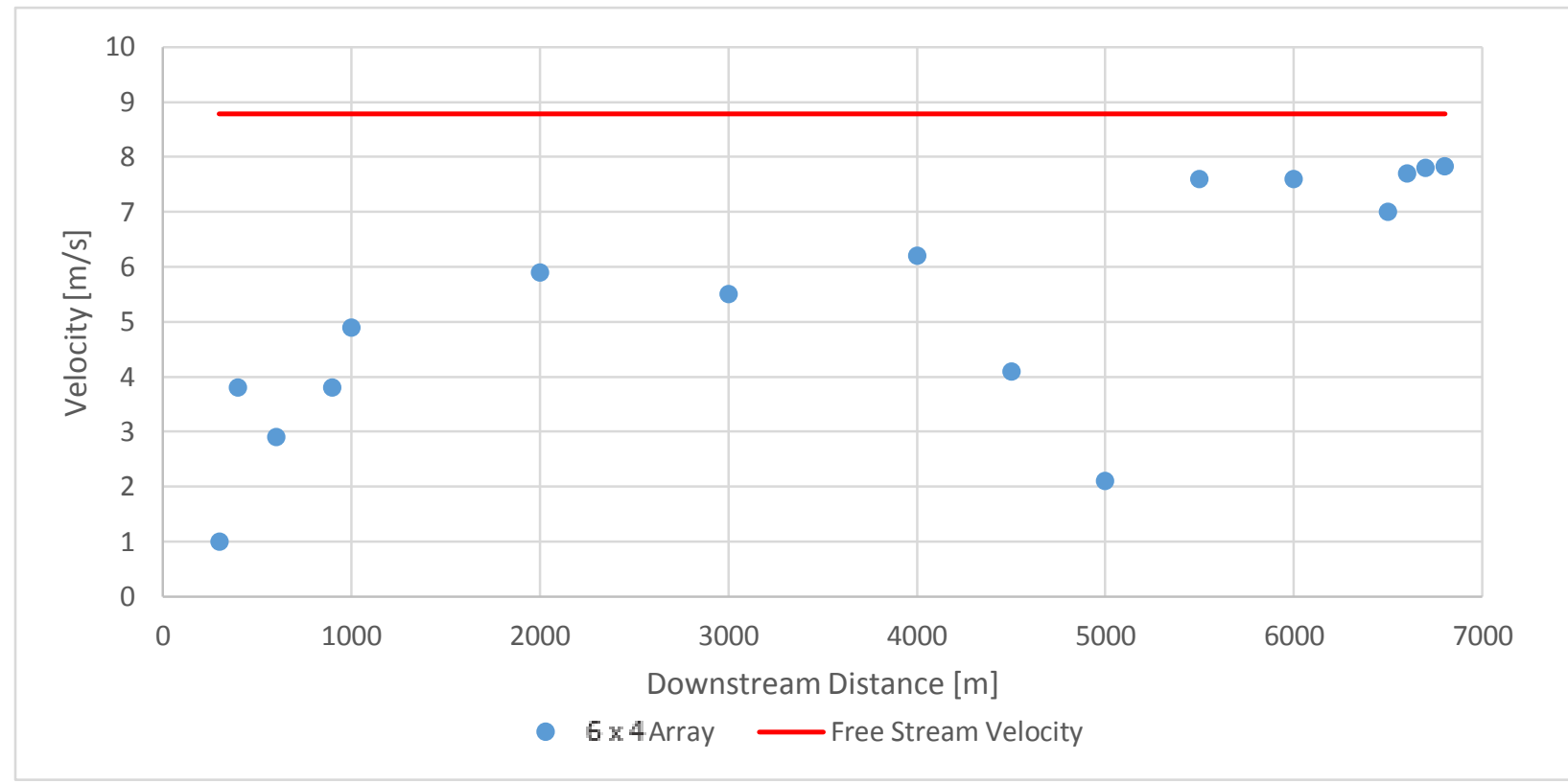

Figure 7.1 Average Wake Velocity across Rotor Position behind Array 1 from Simulation Results

The wake of the leftmost column of Array 2 (Figure 5.3) was not situated in the wake of Array 1 , and so the wake velocity of this column was analyzed to assess the effect on wake recovery of adding more rows. The wake recovery rate of this column of the $8 \times 5$ array was slightly slower than the wake recovery rate of the $6 \times 4$ array. This data is plotted in Figure 7.2. At $3 \mathrm{~km}$ downstream, the differences in the average wake velocity between the two arrays are pronounced with the $6 \times 4$ array wake reaching approximately $5.5 \mathrm{~m} / \mathrm{s}$ and the $8 \times 5$ array wake reaching approximately $1.8 \mathrm{~m} / \mathrm{s}$. This difference in velocity deficits between the two arrays become less, farther downstream. At $4.5 \mathrm{~km}$ downstream, the average wake velocity in the $8 \times 5$ array is only $0.4 \mathrm{~m} / \mathrm{s}$ slower than the $6 \times 4$ array at the same point in the flow field. The turbulent inflow to the $8 \times 5$ array due to the upstream array's wake effect may be causing the wake to recover more 
rapidly than if the $8 \times 5$ array was simulated under a steady inflow condition, as turbulence promotes momentum transfer into the wake.

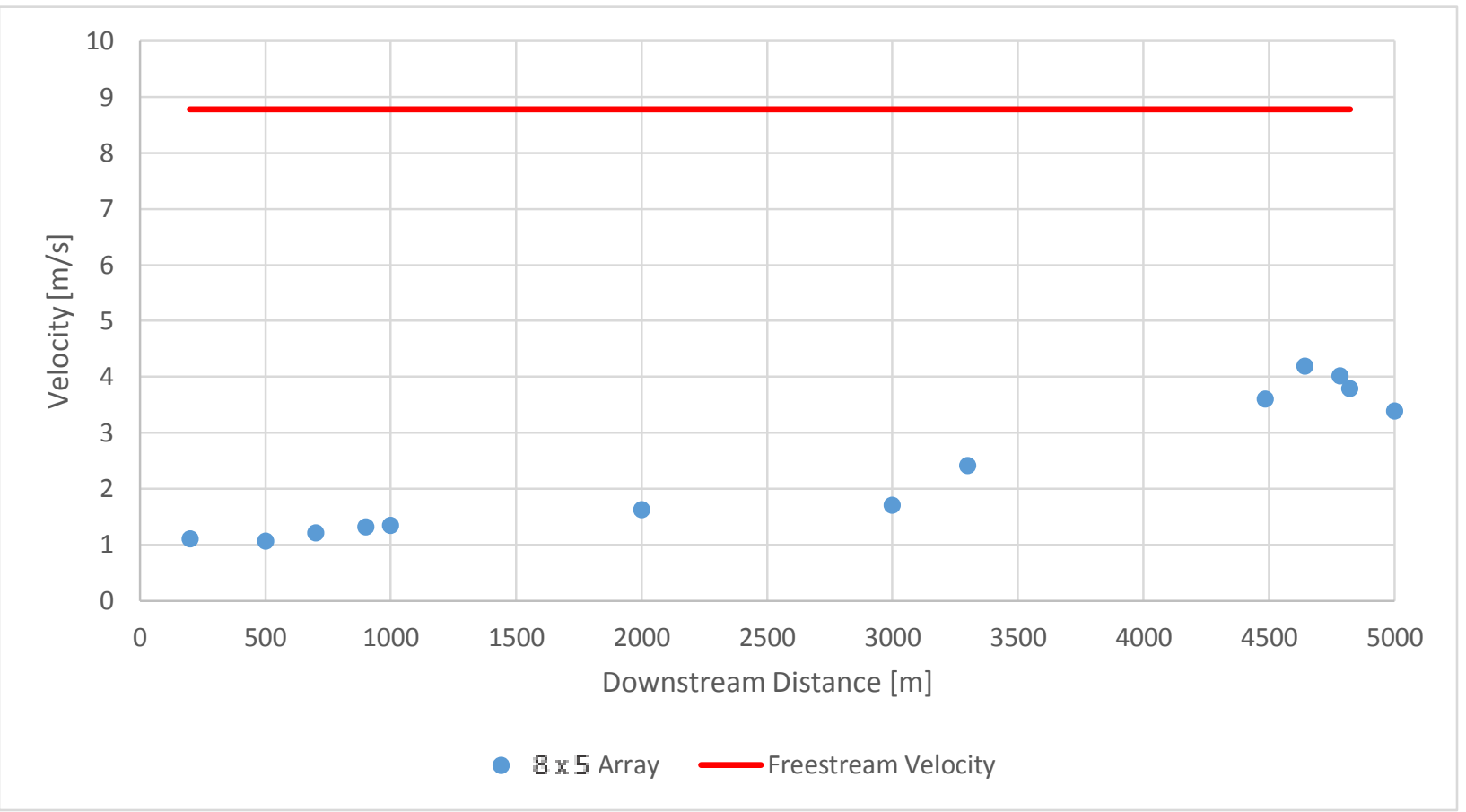

Figure 7.2 Average Wake Velocity across Rotor Position behind Array 2 from Simulation Results

There was evidence of a cumulative wake effect in the simulation within an array. From the most upstream row's wake, row 1, to after the most downstream row in Array 1, the normalized wake velocity across the rotor diameters decreased from $8.4 \mathrm{~m} / \mathrm{s}$ to $0.89 \mathrm{~m} / \mathrm{s}$ in the $6 \mathrm{x} 4$ array. These velocity deficits within Array 1 are plotted in Figure 7.3. 


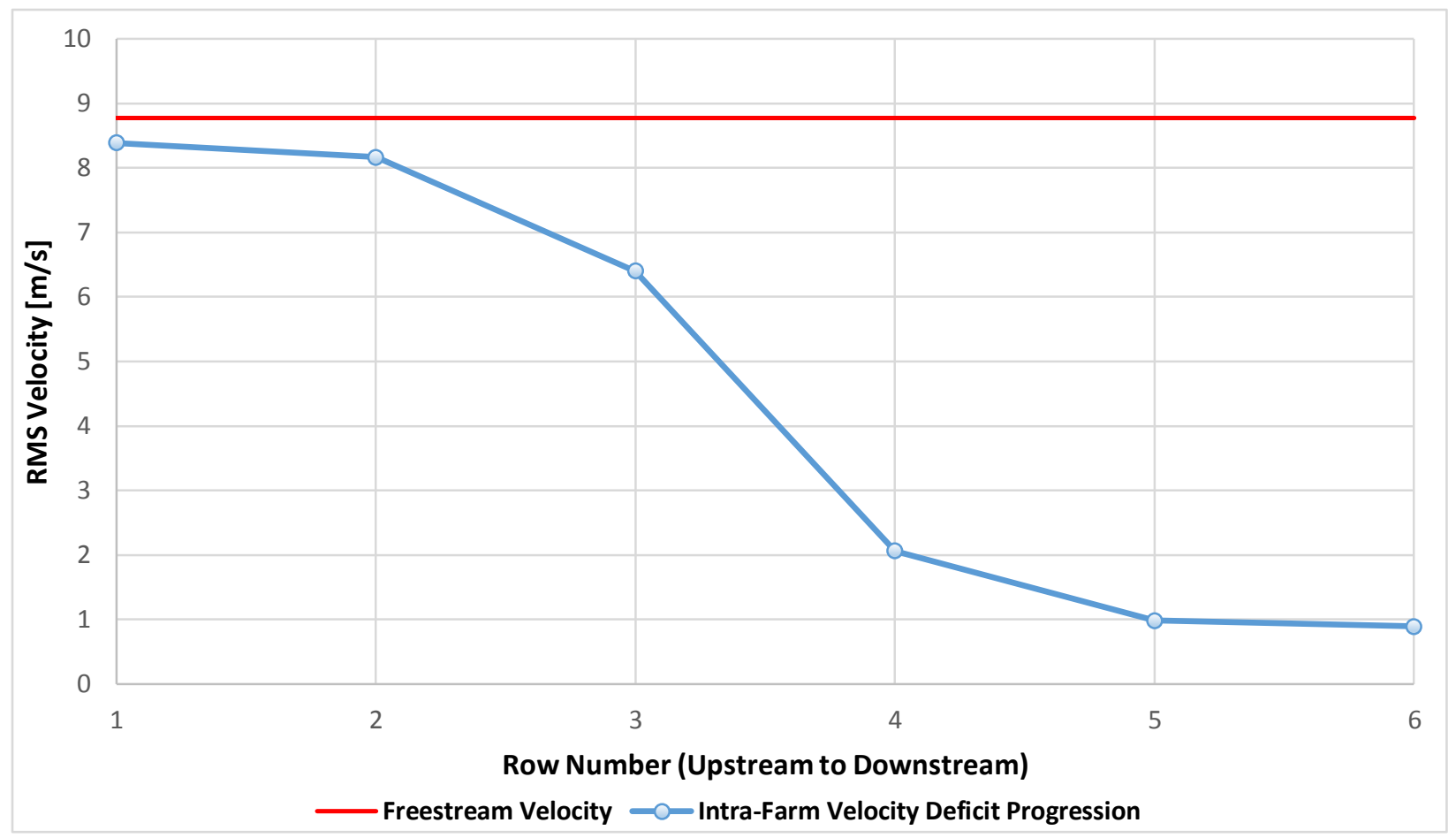

Figure 7.3 Cumulative Wake Effect inside Array 1 from Simulation Results

The cumulative wake effects from both arrays are pictured in Figure 7.4 as the velocity deficit becomes greater in downstream rows. Stretching and merging of vortex cores, results in a re-organization of the overall wake structure. Effects of this re-organization can be seen in Figure 7.4 as there are subtle differences that occur along the stream wise direction in the wake. Lateral spreads of the column wakes increased, and wake meandering periodically occurred downstream from the arrays. Entire wakes of individual columns of turbines periodically drifted due to the motion of large scale eddies in the flow. Bands of significantly increased velocity coincided with the lateral shifting behavior of the wakes. Meandering is known to diminish the overall deficit of a wake [53], and could have been the cause of the bands of increased velocity in the simulation. An additional cause could be due to the constructive interference incurred from the interaction between the streamlines of the weak, periodical vortex cores that are still present in the far wake. 


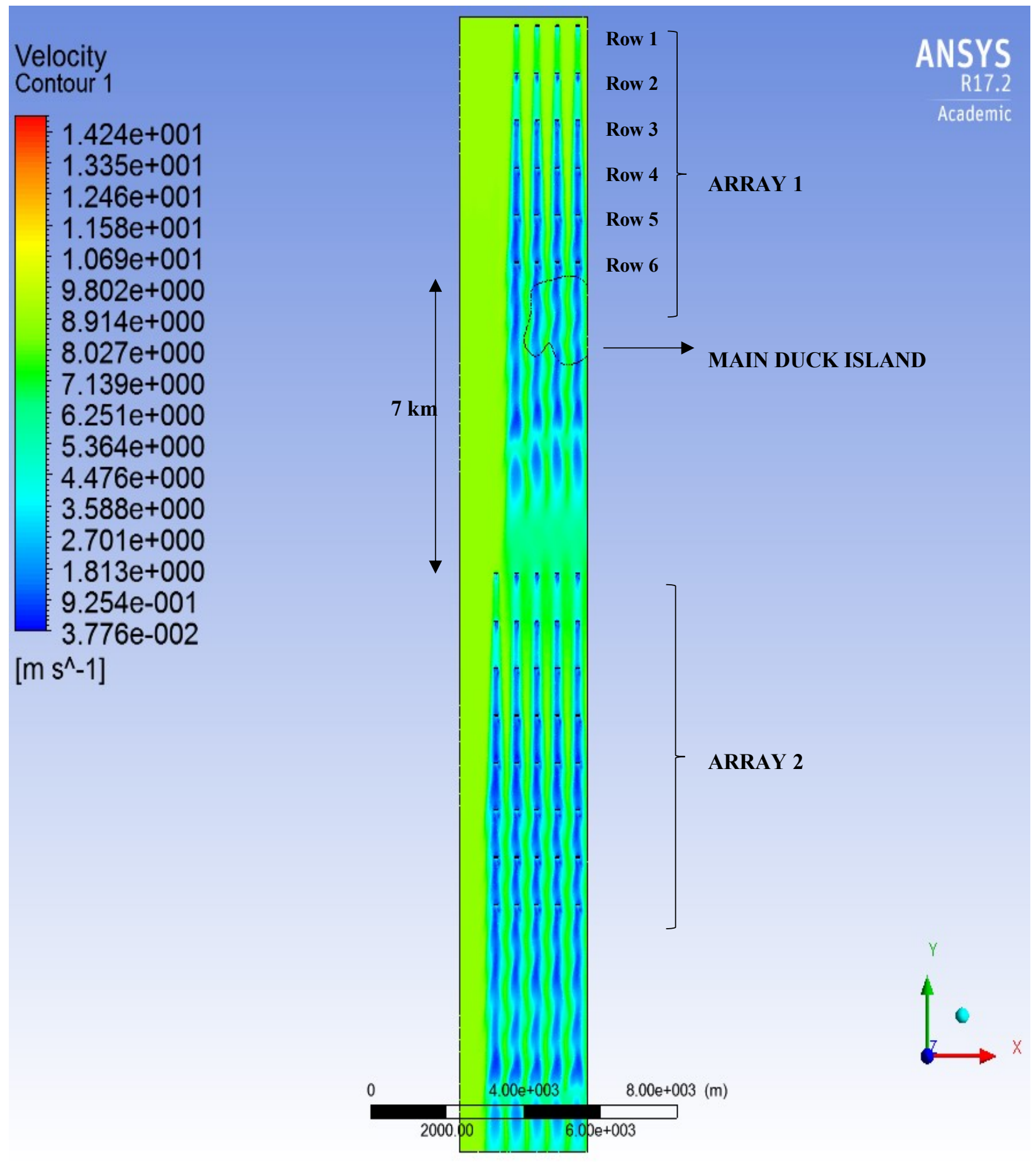

Figure 7.4 Cumulative Wake Effect Velocity Contour Plot from Simulation Results

Figure 7.5 plots the turbulent kinetic energy at hub height over the wake of a turbine behind the sixth row in the first column of Array 1 in Figure 7.4 across the rotor. Swells in turbulent 
kinetic energy coincide with surges in velocity present in the velocity plot in Figure 7.1.

Recovery rate of the wake is most rapid from $5000 \mathrm{~m}$ to $5500 \mathrm{~m}$ downstream of Array 1, as shown in the velocity plot of Figure 7.1. This trend coincides with a high swell of turbulent kinetic energy near $5000 \mathrm{~m}$. This relationship between turbulent kinetic energy in the flow field with a heightened velocity recovery rate is expected as wake recovery is catalyzed by turbulent kinetic energy extracted by large eddies from the mean flow. At $6000 \mathrm{~m}$ downstream, where the wake recovery rate is slowed and the velocity deficit is largely recovered, the turbulent kinetic energy has completely dissipated.

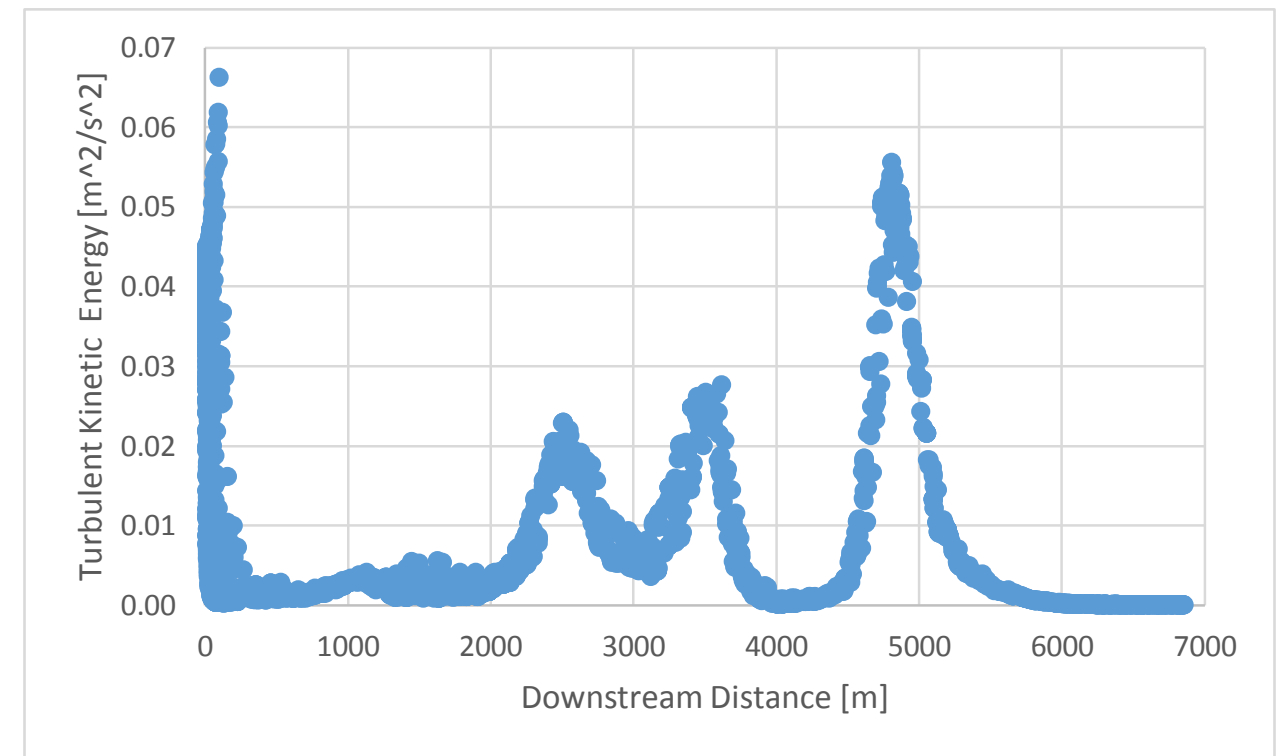

Figure 7.5 Turbulent Kinetic Energy in the Wake of a Single Turbine behind the Sixth Row, in the First Column

\subsection{Single Array Configuration Simulation (7 x 4)}

The boundary conditions in the simulation of the $7 \times 4$ array were the same as in the two-array simulation. Simulation results show that wind velocity would be completely recovered compared to that of the freestream velocity at approximately $13 \mathrm{~km}$ behind the last row of the $7 \mathrm{x} 4$ array. Figure 7.6 is a plot of the normalized velocities over the rotor diameter range for increasing distances behind the $7 \times 4$ array. In this simulation, $89 \%$ recovery is achieved at $9 \mathrm{~km}$ 
downstream from the array and from this point forward, wake velocity steadily increases. After 7 $\mathrm{km}$ downstream from the array, wake velocity recovery rate is reduced. This is because the large eddies that were present further upstream in the wake are severely dissipated near $6 \mathrm{~km}$ downstream, resulting in less mixing to transfer momentum from the freestream flow into the wake. This behavior will be described later in this section through the turbulent kinetic energy plot in Figure 7.8. The 6 row array described in Section 7.1, simulated under the same inlet wind speed, was $89 \%$ recovered at $6.8 \mathrm{~km}$ downstream from the $6 \times 4$ array.

Figure 7.7 illustrates the cumulative wake effect, which shows lateral spreading of the individual wakes as well as meandering. As in the aforementioned analysis on the two array

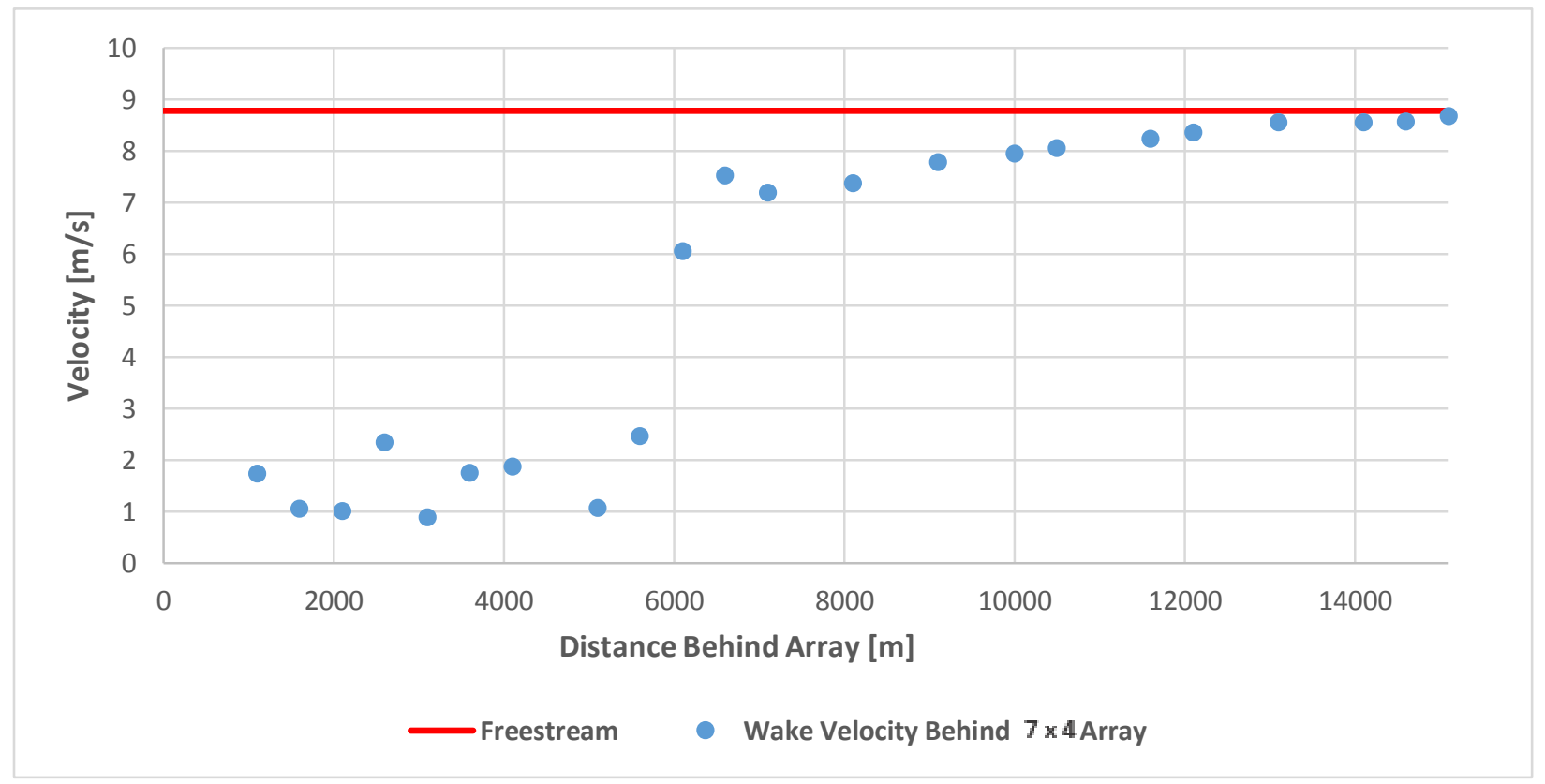

Figure 7.6 Average Wake Velocity across Rotor Position behind 7 x 4 Array from Simulation Results

simulation, there exists thin bands where a slight increase in velocity occurs, rapidly followed by 
a decrease and then a significant increase. This occurrence is most pronounced from $5000 \mathrm{~m}$ to $6300 \mathrm{~m}$ downstream from the array.

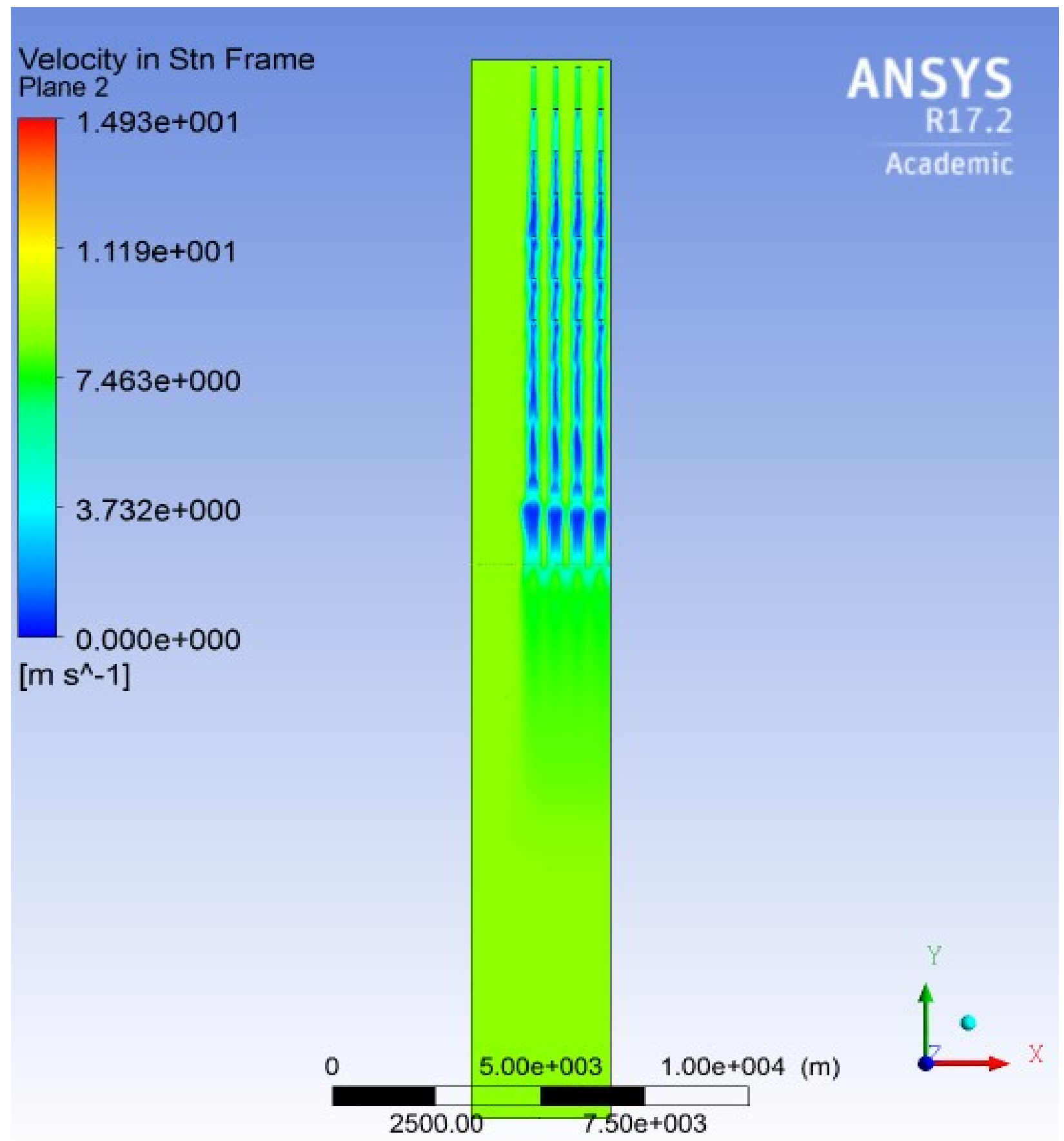

Figure 7.7 Cumulative Wake Effect Velocity Contour Plot for 7 x 4 Array from Simulation Results 
In the wake plots of the two aforementioned simulations, there exists distinct phases of velocity recovery rates. Heightened recovery rates coincide with heightened turbulent kinetic energy levels. Figure 7.8 plots the turbulent kinetic energy for the $7 \times 4$ case at hub height, across the rotor in the wake of turbine in the seventh row of the first column. Near $6000 \mathrm{~m}$, the energy is completely dissipated; which is near the point in which velocity recovery rate slows down.

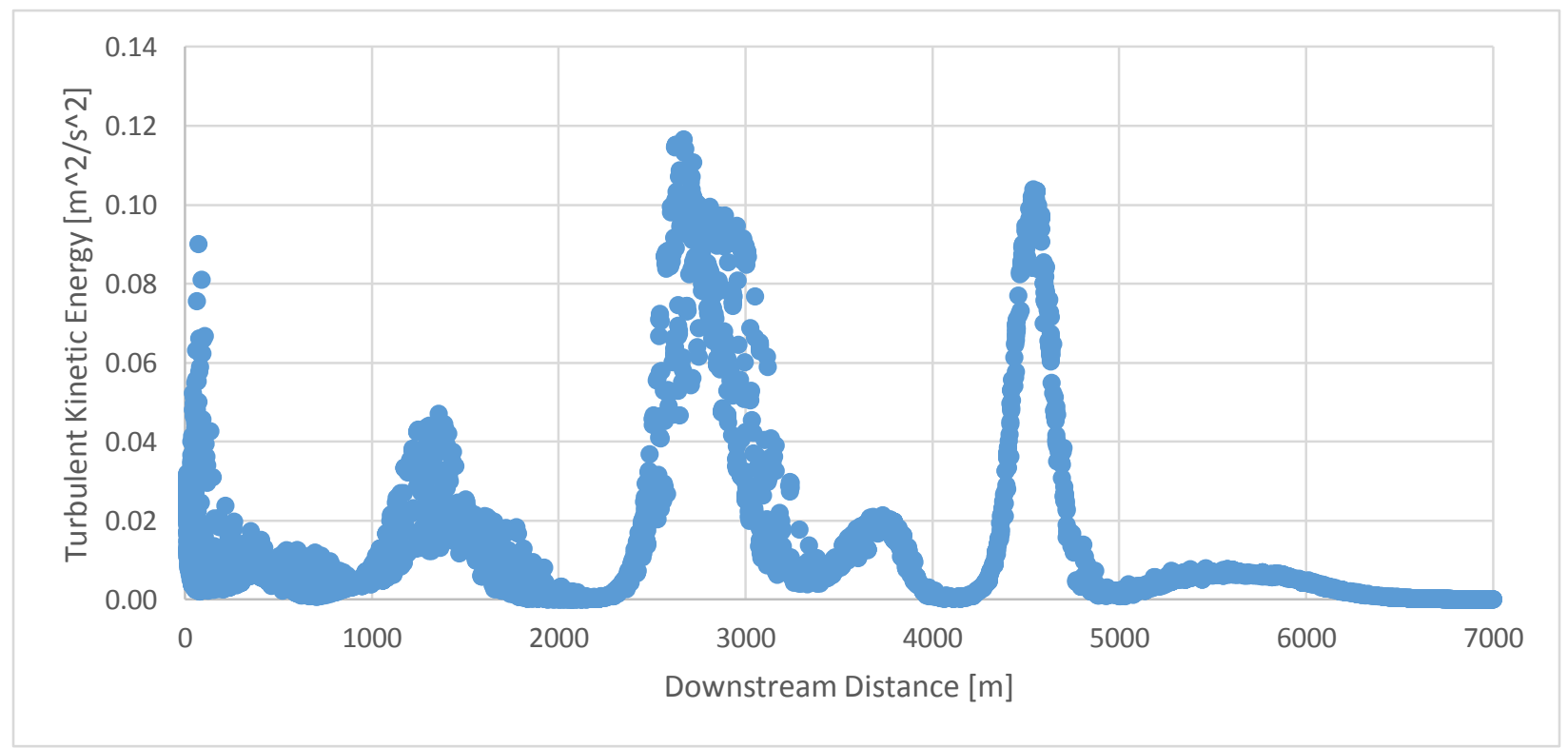

Figure 7.8 Typical Turbulent Kinetic Energy in the Wake of a Single Turbine behind the Seventh Row, in the First Column

\subsection{Increased Inlet Speed for Single Array Simulation ( 7 x 4)}

The sensitivity of wake recovery length to increased inlet wind speed was analyzed for the $7 \mathrm{x}$ 4 array by increasing the inlet wind velocity condition by $10 \%$ to $9.66 \mathrm{~m} / \mathrm{s}$. All other parameters were kept the same as the $7 \times 4$ array case described in Section 7.2. The point in which the average wake velocity was approximately $89 \%$ recovered to the freestream velocity occurred at $10,250 \mathrm{~m}$ downstream from the array; $3,450 \mathrm{~m}$ farther downstream than the lower inlet speed case. Freestream velocity was regained in the wake near $16 \mathrm{~km}$ downstream from the array; $3 \mathrm{~km}$ farther than the lower inlet speed case. This is a $23 \%$ increase in wake recovery length. Figure 7.9 is a plot of the normalized velocities over the rotor diameter range for increasing distances 
behind the $7 \times 4$ array with an increased inlet speed compared to the previously described simulations of Section 7.1 and 7.2.

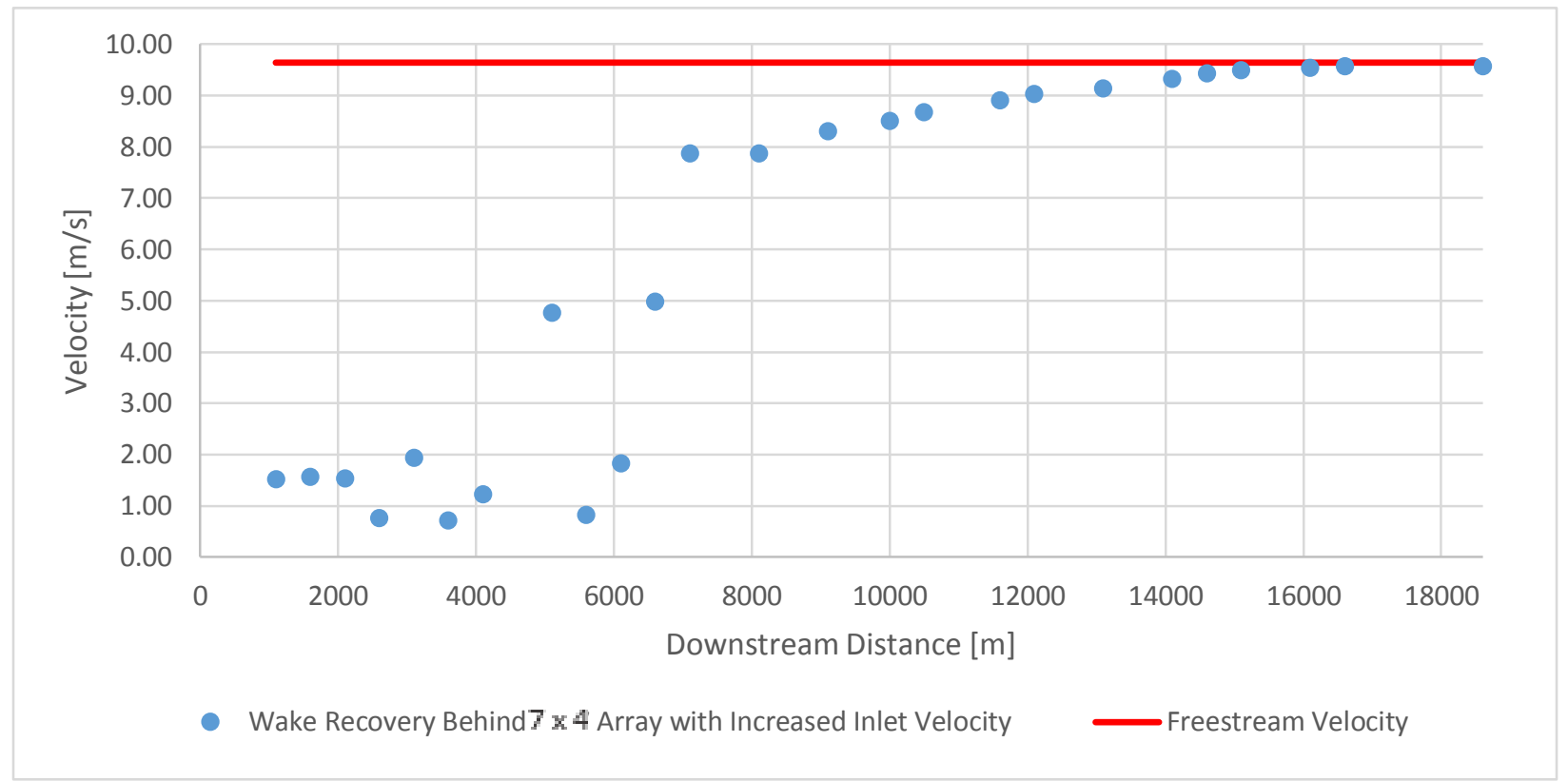

Figure 7.9 Average Wake Velocity across Rotor Position behind 7 x 4 Array with Increased Inlet Wind Speed from Simulation Results

As in the cases of the aforementioned array simulations, wake meandering was present in the increased inlet speed simulation. Bands of increased velocity occur in cycles that coincide with a change in lateral direction of the individual column wakes. The velocity contour plot of the entire array simulation is shown in Figure 7.10. 


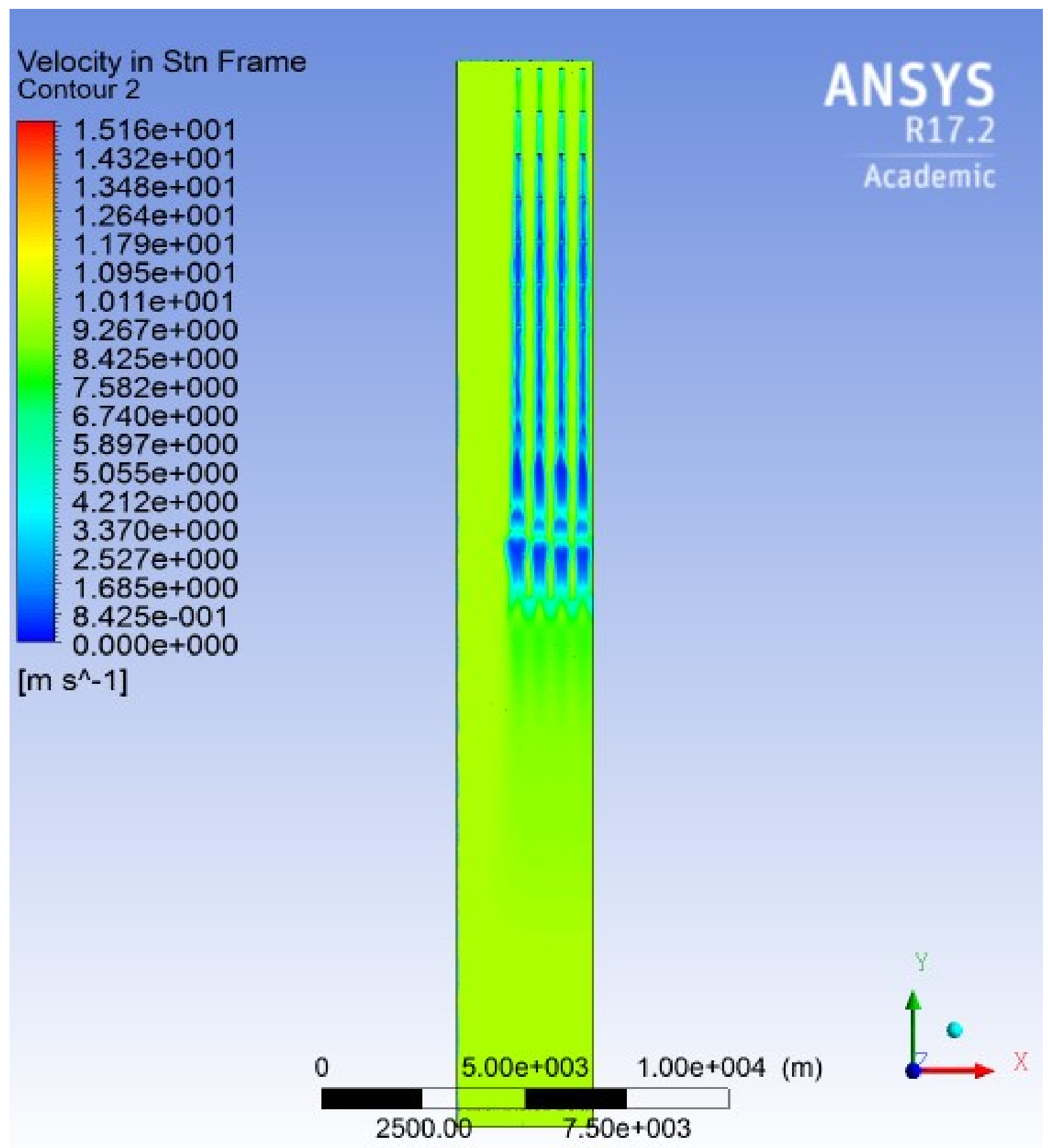

Figure 7.10 Cumulative Wake Effect Velocity Contour Plot for 7 x 4 Array with Increased Inlet Speed from Simulation Results 


\subsection{Influence of Rotor Geometry on Wake Recovery Distance}

The effect of rotor diameter size on the recovery distance of a wake was analyzed by simulating a single turbine of $154 \mathrm{~m}$ in diameter, the same size of the Siemens $6 \mathrm{MW}$ wind turbine. Geometry of the Sandia 3 MW used in the array simulations was scaled up to the larger size for this simulation. A comparison simulation was also ran with Sandia $3 \mathrm{MW}$ rotor size which was $105 \mathrm{~m}$ in diameter. Both cases were simulated with the same grid density and time step for 800 seconds. Velocity values from each simulation were extracted from the horizontal plane, behind the rotor at hub height. The velocity values were averaged over the diameter of the rotor at distances downstream from the rotor and plotted in Figure 7.11 and Figure 7.12. The differences between these two results are minimal in the stream wise direction at hub height. In both cases, by $5000 \mathrm{~m}$ downstream from the rotor, the wake velocity of the turbine is approximately $90 \%$ recovered at an average velocity of $7.9 \mathrm{~m} / \mathrm{s}$ across the diameter of the rotor.

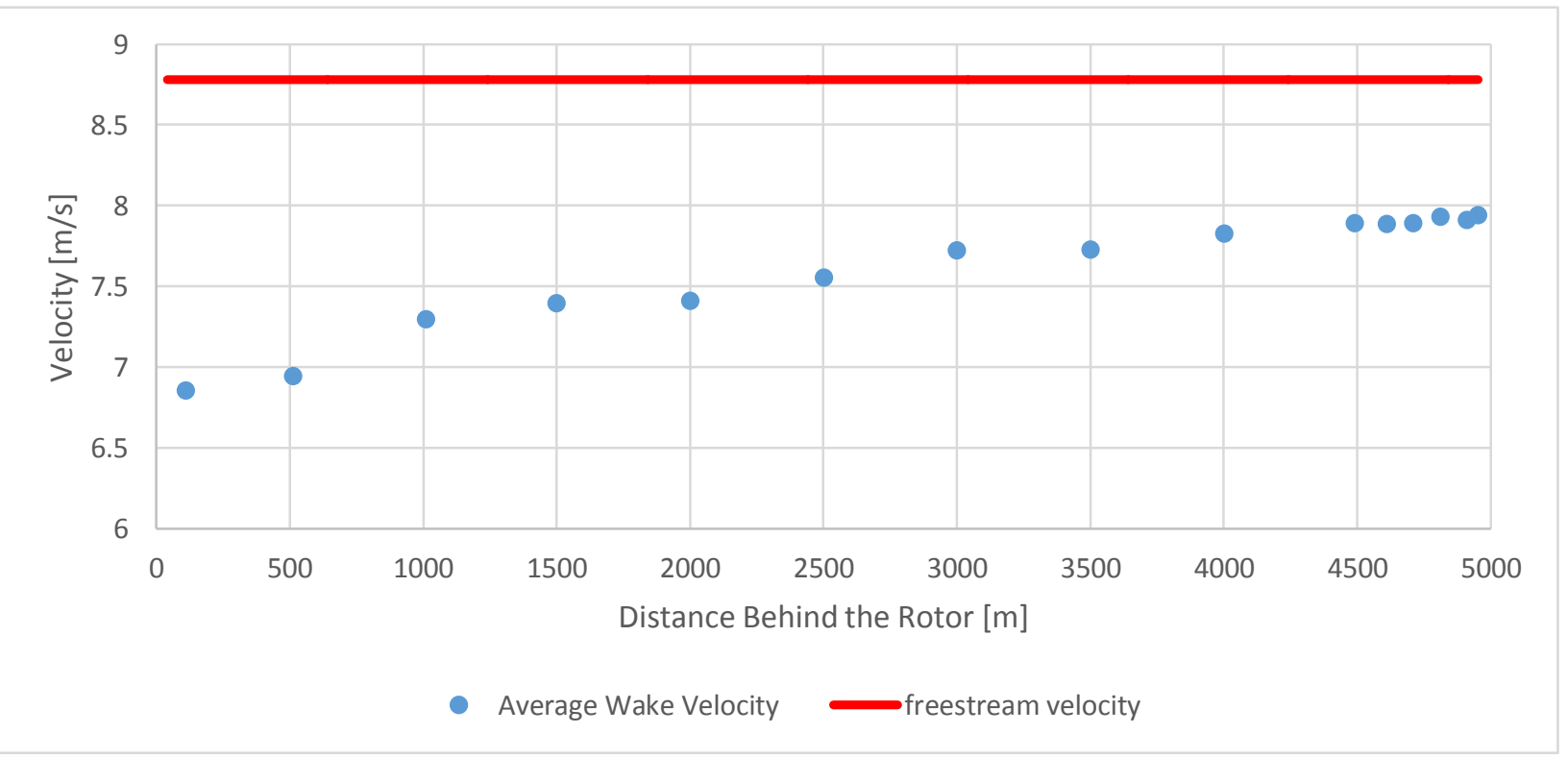

Figure 7.11 Average Wake Velocity across Rotor Position behind Large Rotor (154 m) from Simulation Results 
Significant differences between the simulations are seen near $200 \mathrm{~m}$ downstream where the minimum velocity is lower by approximately $1.3 \mathrm{~m} / \mathrm{s}$ in the small rotor diameter simulation.

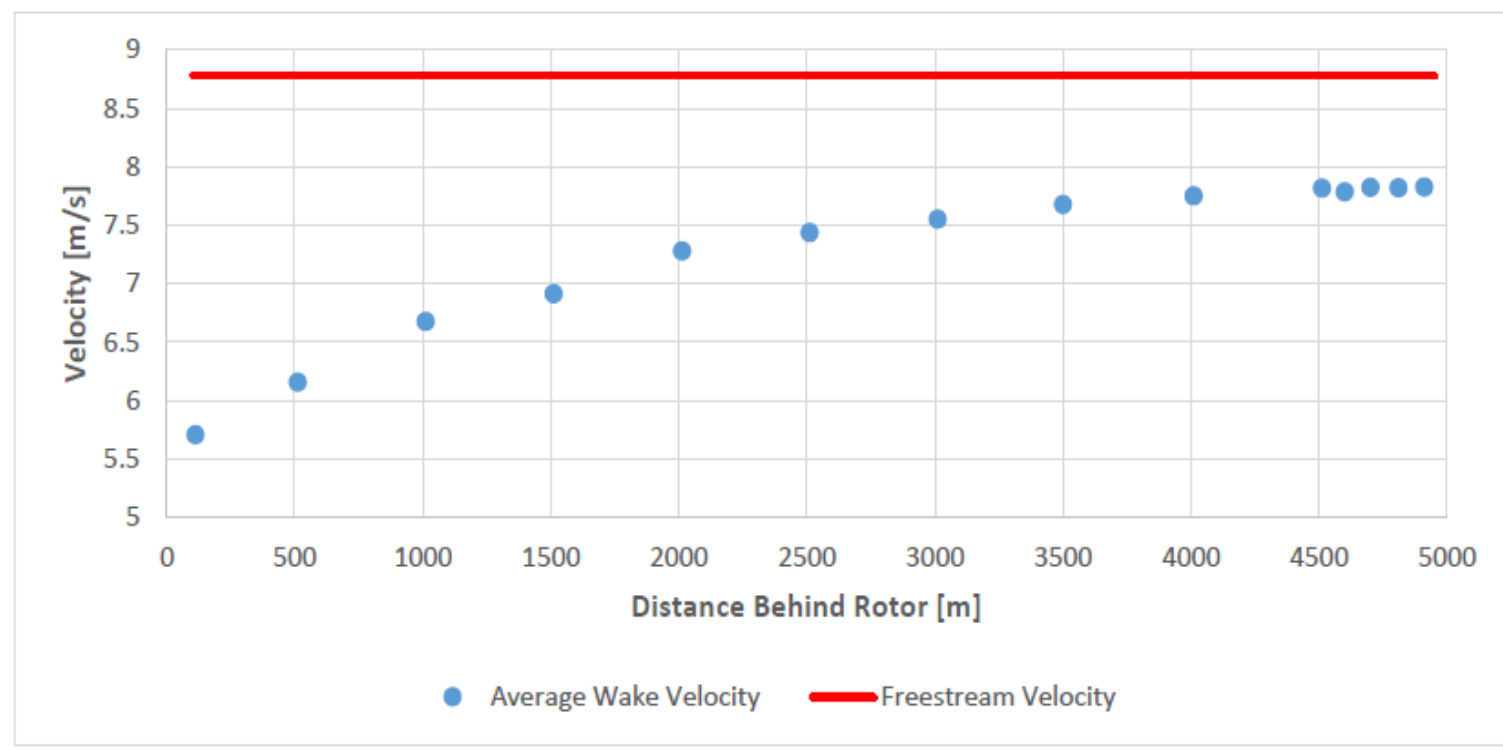

Figure 7.12 Average Wake Velocity across Rotor Position behind Small Rotor (105 m) from Simulation Results

\subsection{Experimental Validation}

Experimental testing was done to validate the CFD modelling techniques used to simulate the offshore arrays. Wind tunnel testing was done on a $25 \mathrm{~cm}$ diameter wind turbine with an Eppler E387 blade design. A hot wire anemometer was used to characterize the flow velocity in the wake of the turbine. This wind tunnel model was replicated in ANSYS CFX and simulated under the same boundary conditions as the experimental test. Hot wire probing was done at hub height of the turbine across the width of the wind tunnel. This process was repeated at 6 different downstream locations, spaced $6 \mathrm{~cm}$ apart. At each probing location in the test section, 65,536 voltage differential readings were collected. The average of these readings was taken for each data collection point in the wind tunnel. All of the averages were then fit to a calibration curve, relating the average voltage differential reading to the velocity. To compare these experimental velocity values to the CFD simulation, the velocities from the grid points in the simulation which 
corresponded to the probing locations in the wind tunnel test were extracted. Percent differences between the velocity values of each experimental data point and corresponding CFD grid point were calculated. The average of the individual absolute percent differences was calculated for each of the 6 rows and also for the entire data set. The cumulative average of the absolute row percent differences between the CFD model data and experimental data was $9.53 \%$. The experimental results indicate that the ANSYS CFD model is accurate and this provides confidence in the meshing scheme, geometry, time step and turbulence model used to simulate Array 1 and Array 2. Experimental errors could have been incurred by probe interference with the flow field, small vibrations in the probe support arm from the wind force as well as through fluctuations in room air temperature of the lab [112]. Table 7.1 displays the cumulative and individual row percent differences between the experimental and CFD data sets.

Table 7.1 Percent Differences; Experimental vs. CFD Simulation Data

\begin{tabular}{|r|r|r|}
\hline Row behind turbine & & Average Error For Each Row Between CFD and Experimental Data (\%) \\
\hline $\mathbf{1}$ & 12.2 \\
$\mathbf{2}$ & 8.6 \\
$\mathbf{3}$ & 10.05 \\
$\mathbf{4}$ & 6.9 \\
$\mathbf{5}$ & 8.38 \\
$\mathbf{6}$ & 11.05 \\
\hline TOTAL AVERAGE & $\mathbf{9 . 5 3}$ \\
\hline
\end{tabular}

As stated in the above, at each probing location in the wind tunnel test section, 65,536

samples were collected. Within each set of data samples, the voltage differential readings fluctuated, largely due to turbulence. The ranges of these fluctuations were calculated in Matlab for the bulk of the data lying within $+/-1$ standard deviation of the mean. Sample probing locations were selected from behind the hub of the turbine and locations on each side of the turbine in the freestream. Fluctuation ranges of these data collection points were plotted in 
Figure 7.13 for rows $1,1.5$ and 2 rotor diameters downstream from the turbine. Locations of the sample data points plotted are depicted in Table 7.2 by the grey shaded regions.

Table 7.2 Sample Data Point Locations

\begin{tabular}{|c|c|}
\hline$x / D$ & Rotor D \\
\hline 0.5 & \\
\hline 1 & \\
\hline 1.5 & \\
\hline 2 & \\
\hline
\end{tabular}

Fluctuations in the sample data from the aforementioned points are represented by the black error bars in Figure 7.13. For comparison, the corresponding data points from the CFD simulation are superimposed on the error bars. Of this sample data set, all simulation points fell within the experimental data ranges except for the point directly behind the turbine hub at 1D. Agreement between the two data sets becomes better, farther downstream.

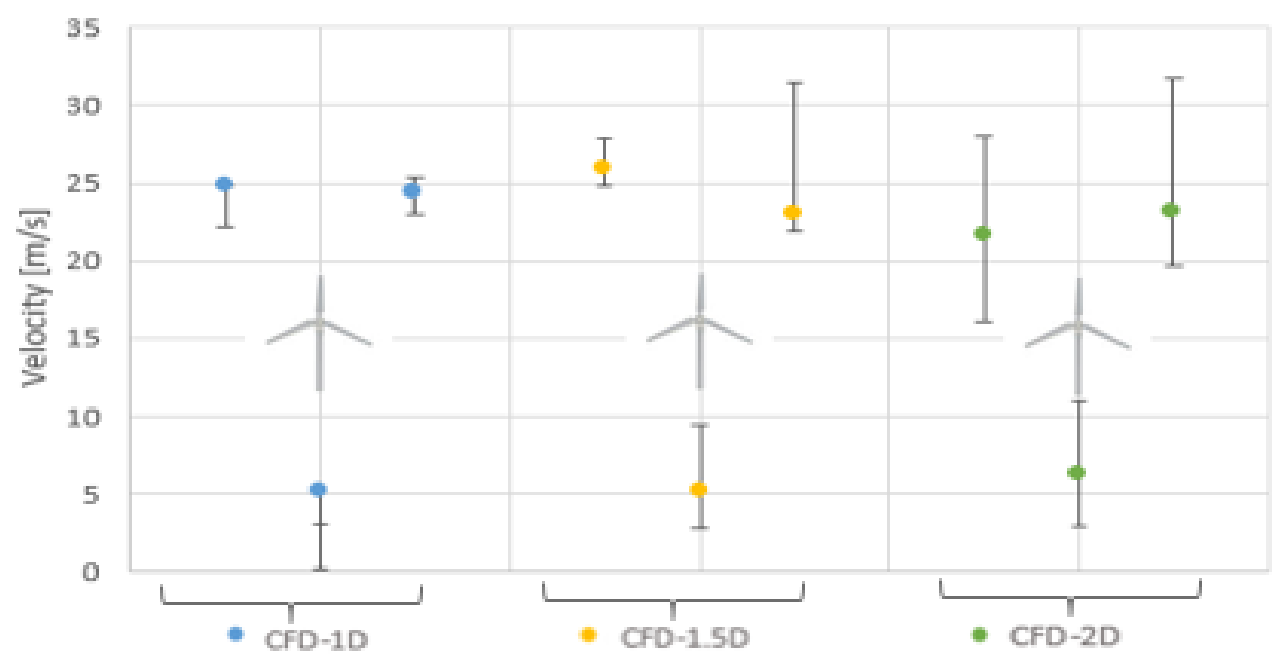

Figure 7.13 Experimental Data Ranges Compared with CFD Data. (Image source: [122]) 


\subsection{Convergence Levels}

In the simulations of this research, the residual values and imbalance percentages indicated that acceptable convergence levels were obtained after 2000 iterations. The convergence levels of the simulations verified that the results are a good approximate solution to the governing CFD equations under the initial and boundary conditions of the simulations. Convergence is quantitatively measured by the value of the residuals in the equations and the net flux through the stationary and rotational domains. ANSYS CFX iteratively resolves the non-linear flow equations of mass, momentum and turbulence. For each cell in the domain, a normalized residual value is calculated for every equation in the $\mathrm{u}, \mathrm{v}$ and $\mathrm{w}$ directions. Section 3.3.3 described the concept of residuals. Tighter convergence levels are indicated by lower residual values. An initial guess was input into the transient simulation in the form of the steady-state simulation. As the transient model stepped through time, the residual values were lowered until the desired target for error acceptance was achieved. This occurred after 800 seconds with a time step of 0.4 seconds, or 2000 iterations.

Percentages for the flux of total mass flow rate between the stationary domain inlet and outlet, and across the rotational domains, were $1.7 \mathrm{E}-3 \%$ and $1.6 \mathrm{E}-6 \%$ respectively. This is well under the accuracy criteria of $1 \%$. The residual value averages across the flow field in the direction of the mean flow were 4.22E-06 and 1.92E-05 for the Mass and Momentum equations respectively. For the k- $\omega$ turbulence model equations evaluating turbulent kinetic energy (k) and frequency $(\omega)$ over the entire flow field, the residuals were $2.35 \mathrm{E}-05$ and 9.23E-06 respectively. The aforementioned residuals lie near the ranges of well and tightly converged numerical solutions (1e-5 and 1e-6). Refer to Table 7.3 for a detailed summary of all imbalances and residual values in the flow field for the respective domains. 
Table 7.3 Convergence Analysis

\begin{tabular}{|l|r|}
\hline Parameter & Value \\
\hline RMS k-Turbulence Kinetic Energy & $2.35 \mathrm{E}-05$ \\
RMS O-Turbulence Frequency & $9.23 \mathrm{E}-06$ \\
\hline RMS Wall scale & $1.75 \mathrm{E}-06$ \\
\hline RMS P-Mass & $4.22 \mathrm{E}-06$ \\
RMS U-Momentum & $1.44 \mathrm{E}-05$ \\
RMS V-Momentum & $1.92 \mathrm{E}-05$ \\
RMS W-Momentum & $1.1 \mathrm{E}-05$ \\
\hline Rotational Domains P-Mass Imbalance & $0.0000016 \%$ \\
Rotational Domains U-Momentum Imbalance & $0.0001520 \%$ \\
Rotational Domains V-Momentum Imbalance & $0.0000033 \%$ \\
Rotational Domains W-Momentum Imbalance & $0.0005370 \%$ \\
\hline Stationary Domain P-Mass Imbalance & $0.0017 \%$ \\
Stationary Domain U-Momentum Imbalance & $0.0079 \%$ \\
Stationary Domain V-Momentum Imbalance & $0.0312 \%$ \\
Stationary Domain W-Momentum Imbalance & $0.0034 \%$ \\
\hline
\end{tabular}




\section{Conclusion and Future Work Recommendations}

The offshore array simulations indicate that in regions far from the arrays, wake effects are present, an important consideration for offshore wind farm development. The two-array simulation revealed that a spacing of $7 \mathrm{~km}$ is not quite adequate for the placement of the proposed arrays in the Great Lakes. At $6.8 \mathrm{~km}$ downstream of the $6 \times 4$ array, the fluid velocity is approximately $89 \%$ recovered. At $9 \mathrm{~km}$ downstream of the $7 \times 4$ array with the same inlet condition as in the $6 \times 4$ array simulation, the fluid velocity is approximately $89 \%$ recovered, revealing that adding rows to an array delays wake recovery. Freestream velocity levels were regained at approximately $13 \mathrm{~km}$ downstream from the $7 \times 4$ array. These results predicted a $32 \%$ increase in wake recovery distance when a row was added to the $6 \times 4$ array. Large eddies caused lateral movement in a periodic fashion which coincided with bands of increased velocity. The dissipation of large eddies near $6 \mathrm{~km}$ downstream of the arrays is responsible for the slowed recovery rate of the velocity deficit in the wakes after $89 \%$ recovery was achieved.

The increased inlet speed simulation revealed that a $10 \%$ increase in freestream velocity has a significant impact on the wake recovery length. In this simulation, the inlet speed was increased from $8.78 \mathrm{~m} / \mathrm{s}$ to $9.65 \mathrm{~m} / \mathrm{s}$. Results revealed meandering patterns that coincided with bands of increased velocity as in the other array simulation. The wake velocity returned to freestream levels at approximately $16 \mathrm{~km} ; 3 \mathrm{~km}$ further downstream from the point in which the lower inlet speed simulation wake was recovered.

In the SAR wake study of the Horns Rev, Nysted and Sheringham Shoals offshore wind farms described in Section 4.1, wake recovery length was recorded to be between 5 and $30 \mathrm{~km}$ depending on freestream conditions, atmospheric stability and turbines in operation. The wake 
recovery lengths for the simulations of this research show comparable ranges of recovery lengths for arrays of roughly the same sizes as the subjects in the aforementioned SAR study [80] [6]. In the results of the Wyoming full rotor array simulation [88], significant cumulative wake effects occurred in the downstream rows of the simulated array. This trend occurred in all of the array simulations of this research.

A single turbine simulation was run to look at the impact of rotor diameter size on wake recovery length. Differences between the simulation results in the wake velocity profile were apparent in the near wake region, but at $5 \mathrm{~km}$ downstream, the velocity had recovered to the same level in both cases.

Experimental wind tunnel tests were compared to a small-scale CFD simulation, and produced an average absolute percent difference of $9.53 \%$ from the CFD results. These results gave confidence to the CFD modelling techniques employed in the full-scale simulation. The CFD numerical results were verified through mesh and time step sensitivity studies, as well as the small residual values and imbalance percentages for the large-scale simulation. Residual RMS values for the two array simulation were $4.22 \mathrm{E}-06$ and $1.92 \mathrm{E}-05$ for the mass and momentum equations respectively. For the k- $\omega$ turbulence model equations over the entire flow field, the residuals were $2.35 \mathrm{E}-05$ and $9.23 \mathrm{E}-06$ respectively. These values indicate that the final solution was tightly converged. Imbalance percentages were also well under the convergence criterion of $1 \%$ for the stationary and rotational domains. The aforementioned RMS residual and imbalance levels were also achieved in the alternate configuration and sensitivity analysis simulations. Sensitivity studies were also used to verify the CFD modelling techniques. Results from these studies showed a lack of variation between simulations with different time step inputs. More sensitivity was shown through the grid density study. 
A natural progression of this work would be to gain access to more powerful computing resources to simulate the arrays with the full number of turbines that will be constructed in the proposed Trillium Wind Power arrays. This would eliminate the need for the symmetry boundary condition and would provide an interesting comparison study. Increasing the initial freestream velocity and the number of rows in an array caused a notable delay in wake recovery length of the CFD simulations. Performing additional array simulations to see the effects of a wider range of inlet wind speeds as well as a greater number of rows would be useful. Using open source codes may be a more efficient way to simulate larger wind farms.

Scaling up the experimental test case to simulate an entire array of wind turbines, and comparing the results with a CFD simulation could also provide valuable insight. CFD array models could be informed through a series of wind tunnel tests using full arrays. These wind tunnel tests could serve to validate the CFD model using Bayesian analysis. The rotor diameter comparison study of this research could be improved upon by simulating the larger diameter rotor with a more realistic blade geometry profile, rather than simply scaling up the small rotor geometry. 


\section{Appendix A}

Below is the derivation of the large eddy length scale related to the Kolmogorov length scale. Algebraic manipulation of the $\mathrm{k} \sim \varepsilon$ relationship is performed to obtain a relationship between $\mathrm{L}$ and $\eta$. Equation 1 is the expanded form of the $\mathrm{k} \sim \varepsilon$ relationship.

$$
\frac{V_{\text {Int. }}^{3}}{L} \sim \frac{V_{\text {Kol. }}{ }^{3}}{\eta^{4}}
$$

Rearrange Equation 1 to get Equation 2 in terms of L:

$$
L \sim \frac{V_{\text {Int. }}{ }^{3} \eta^{4}}{v^{3}}
$$

Recall that the ratio of $\mathrm{L}$ to $\eta$ is desired. Through algebraic manipulation, Equation 3 is obtained:

$$
\frac{L}{\eta^{4}} \sim \frac{V_{\text {Int. }}^{3}}{V_{\text {Kol. }}{ }^{3}}
$$

The two length scales are now both on the left hand side of the equation, but need to be of the same order. Multiplying both sides of Equation 3 by $L^{3}$ yields Equation 4 .

$$
\frac{L^{4}}{\eta^{4}} \sim \frac{V_{\text {Int. }}{ }^{3} L^{3}}{V_{\text {Kol. }}{ }^{3}}
$$

The right hand side of the equation is the Reynolds number cubed.

$$
\frac{L^{4}}{\eta^{4}} \sim R e^{3}
$$

Reducing the left hand side of the equation to the desired ratio, $\frac{L}{\eta}$, yields Equation 6 . 


$$
\frac{L}{\eta} \sim R e^{3 / 4}
$$




\section{List of References}

[1] US Department of Energy and US Department of Interior Workshop to Inform, "2016 National Offshore Wind Strategy," Consensus Building Institue, 2016.

[2] Z. Wang, "Experimental and Numerical Investigations on the Flow Characteristics of Rotary," Ph.D. dissertation,Dept. of Aero. Eng., Iowa State University, Ames, IA, 2015.

[3] D. Spera and H. Neustadter, "Method for evaluating wind turbine wake effects on wind farm performance," Journal of Solar Energy Engineering, p. 107:240-243, 1985.

[4] R. Barthelmie, S. Frandsen, K. Hansen, J. Schepers, K. Rados and W. Schlez, "Modelling the impact of wakes on power output at Nysted and Horns Rev.," 2009.

[5] EWEA, "Development and Investment Costs of Offshore Wind Power," [Online]. Available: https://www.wind-energy-the-facts.org/development-and-investment-costs-ofoffshore-wind-power.html.

[6] A. Pena Diaz, C. B. Hasager and R. Husson, "Far Wake Wind Field Comparison between Satellite Retrievals and Microscale Model Results," in EWEA Offshore, Copenhagen, 2015.

[7] SIEMENS, "Wind without End," 10 February 2016. [Online]. Available: https://www.siemens.com/innovation/en/home/pictures-of-the-future/energy-andefficiency/sustainable-power-generation-hywind.html. [Accessed 27 March 2017].

[8] M. Geuss, "First offshore wind farm in US waters delivers power to Rhode Island," 13 Dec. 2016. [Online]. Available: http://arstechnica.com/science/2016/12/first-offshore-windfarm-in-us-waters-is-delivering-power-to-rhode-island/. [Accessed 12 Jan. 2017].

[9] CANWEA, "Why Wind Works," [Online]. Available: canwea.ca. [Accessed 263201 ].

[10] A. Morrow, "The Globe and Mail," 04 May 2016. [Online]. Available: http://www.theglobeandmail.com/news/politics/opp-probe-allegations-ontario-liberalgovernment-destroyed-power-project-documents/article29851728/. [Accessed 20 March 2017].

[11] L. Jalali, "Investigation of Effects of Wind Turbine Noise on General Health and Sleep Disturbance," Ph.D Dissertation, University of Waterloo, Waterloo, Ontario, Canada, 2016.

[12] T. Spears, "Ontario's 'gold rush' wind energy plan ignored impact on rural communities, $\begin{array}{llllll}\text { study says," } & 4 & 2 & 2016 . & \text { [Online]. Available: }\end{array}$ http://news.nationalpost.com/news/canada/ontarios-gold-rush-wind-energy-plan-ignoredimpact-on-rural-communities-study-says. [Accessed 273 2017].

[13] D. Ouellette, C. Walker and J. Baxter, "Adding insult to injury: The development of psychosocial stress in Ontario wind turbine communities," Social Science and Medicine, vol. 133, pp. 358-365, 2014.

[14] K. Otter and P. James, "UNBC Research Highlights Bird-Wind Energy Project Interactions," June 2014. [Online]. Available: http://www.unbc.ca/releases/34484/unbcresearch-highlights-bird-wind-energy-project-interactions. [Accessed March 26 2017].

[15] USGS, "Bat Fatalities at Wind Turbines: Investigating the Causes and Consequences," [Online]. Available: https://www.fort.usgs.gov/science-feature/96. [Accessed 203 2017]. 
[16] S. Pratt, "Wind farms causing thousands of bats to die of collapsed lungs annually in Alberta: top bat expert," 19 November 2014. [Online]. Available: http://news.nationalpost.com/news/canada/wind-farms-causing-thousands-of-bats-to-dieof-collapsed-lungs-annually-in-alberta-top-bat-expert. [Accessed 26 March 2017].

[17] Alberta Government Environment and Sustainable Resource Development, "Bat Mitigation Framework for Wind Power Development Wildlife Land Use Guidelines," Government of Alberta, 2013.

[18] Stantec, "Environmental Effects Monitoring Plan for Wildlife and Wildlife Habitat," Prepared for : Suncor Energy Products Inc. Prepared by: Stantec Consulting Ltd., 2014.

[19] CBC new Technology and Science, "Wind turbine noise linked to only 1 health issue annoyance," 9 April 2015. [Online]. Available: http://www.cbc.ca/news/technology/windturbine-noise-linked-to-only-1-health-issue-annoyance-1.3025495. [Accessed 27 March 2017].

[20] CBC news Science and Technology, "Wind turbine noise not linked to health problems, Health Canada finds," 6 November 2014. [Online]. Available: http://www.cbc.ca/news/technology/wind-turbine-noise-not-linked-to-health-problemshealth-canada-finds-1.2826206. [Accessed 27 March 2017].

[21] I. Ruiz and T. Alonso, "Gravity Base Foundations for Offshore Wind Farms," University of Cantabria, 2013.

[22] International Energy Agency, "Technology Roadmap," 2013.

[23] Natural Resources Canada, "Offshore Oil and Gas," 22 August 2016. [Online]. Available: https://www.nrcan.gc.ca/energy/offshore-oil-gas/5835. [Accessed 10 March 2017].

[24] International Joint Commission, "Protecting Shared Resources," IJC, 2017. [Online]. Available: http://www.ijc.org/en_/Protecting_Shared_Resources. [Accessed 9 April 2017].

TransAlta,

"St.Mary,"

2017.

[Online].

Available: http://www.transalta.com/facilities/plants-operation/st-mary/. [Accessed 5 April 2017].

[26] Alberta Agriculture and Forestry, "Irrigation in Alberta," 11 March 2015. [Online]. Available: http://www1.agric.gov.ab.ca/\$department/deptdocs.nsf/all/irr7197. [Accessed 2 April 2017].

[27] "Letter of Intent to Better Utilize the Waters of the St. Mary and Milk Rivers," 2001.

[28] IESO, "Ontario's Power System," 2017. [Online]. Available: http://www.ieso.ca/learn/ontario-power-system/electricity-market-today-andtomorrow\#demandresponse. [Accessed 10 April 2017].

[29] AESO, "ISO Rules; Part 300 System Reliability and Operations; Division 304 Routine Operations; Section 304.3 Wind Power Ramp Up Management," 2015.

[30] International Joint Commission , "Air Quality," IJC, 2017. [Online]. Available: http://www.ijc.org/en_/Air_Quality. [Accessed 9 April 2017].

[31] "Great Lakes Water Quality Agreement 2012," IJC, 2017. [Online]. Available: http://www.ijc.org/en_/Great_Lakes_Water_Quality. [Accessed 9 April 2017].

[32] EWEA, "Delivering Offshore Wind Power in Europe," 2007.

[33] WindEurope, "The European offshore wind industry: Key trends and statistics 2016," WindEurope, 2017. 
[34] J. Wilkes, I. Pineda and G. Corbetta, "Wind energy scenarios for 2020," European WInd Energy Association (EWEA), 2014.

[35] European Wind Energy Association, "Delivering Offshore Wind Power in Europe," 2007.

[36] M. Cini and N. Borragan, "European Union Politics," 5th ed. Oxford: Oxford University Press, 2016.

[37] European Union, "EU institutions and other bodies," 2017. [Online]. Available: https://europa.eu/european-union/about-eu/institutions-bodies_en. [Accessed 2 April 2017].

[38] Wind Europe, "The European offshore wind industry - key trends and statistics 2016," 26 January 2017. [Online]. Available: https://windeurope.org/aboutwind/statistics/offshore/european-offshore-wind-industry-key-trends-and-statistics-2016/. [Accessed 2 May 2017].

[39] WindEurope, "Mission \& Values," WindEurope, 2017. [Online]. Available: https://windeurope.org/about-us/mission-values/. [Accessed 10 May 2017].

[40] Munson, Okiishi, Huebsch and Rothmayer, "Fundamentals of Fluid Mechanics," in Fundamentals of Fluid Mechanics, Wiley, 2013, pp. 485-487.

[41] M. Drela, Fluid Mechanics, Fall 2005- Spring 2006. (Massachusetts Institute of Technology: MIT OpenCouseWare), http://ocw.mit.edu (Accessed [March 3, 2017]). License: Creative Commons BY-NC-SA.

[42] ANSYS Inc., "4.2. Modeling Flow Near the Wall," ANSYS ®, Academic Research, Release 15.0, ANSYS CFX-Solver Theory Guide.

[43] W. Devenport, "“FLOW PAST A CIRCULAR CYLINDER”," 2007.

[44] National Aeronautics and Space Administration (NASA), "SP-367 Introduction to the Aerodynamics of Flight," 1975. [Online]. Available: https://history.nasa.gov/SP367/f48.htm. [Accessed 2 March 2017].

[45] S. Chakraborty, Introduction to Turbulence Modelling, Department of Mechanical \& Engineering, NPTEL, 2010.

[46] D. Medici, "Wind Turbine Wakes - Control and Vortex Shedding," Royal Institute of Technology, Stockholm, Sweden, 2004.

[47] M. Sherry, A. Nemes, D. L. Jacono, H. M. Blackburn and J. Sheridan, "The interaction of helical tip and root vortices in a wind turbine wake," PHYSICS OF FLUIDS, vol. 25, 2013.

[48] B. Johnson, "Computational Fluid Dynamics (CFD) Modelling of Renewable Energy Turbine Wake Interactions, Ph.D. Dissertation, University of Central Lancashire," Preston, England, 2015.

[49] Y. Jin, H. Liu, R. Aggarwal, A. Singh and L. P. Chamorro, "Effects of Freestream Turbulence in a Model Wind Turbine Wake," MDPI, pp. 5,6, 2016.

[50] D. Maniaci, "Wakes and Rotors: The National Rotor Testbed," Sandia National Laboratories, 2014.

[51] R. Stoll, LES of Turbulent Flows: ME EN 7960-003 Lecture, Department of Mechanical Engineering, University of Utah, 2014.

[52] University of Utah Lecture, Length and Time Scales In Turbulent Flows, Lecture 7. 
[53] B. Sanderse, "Aerodynamics of Wind Turbine Wakes Literature Review," Energy Research Centre of the Netherlands.

[54] J. Ainslie, "Calculating the field in the wake of wind turbines," Journal of Wind Engineering and Industrial Aerodynamics, p. 27:213-224, 1988.

[55] M. Liu, M. Yocke and T. Myers, "Mathematical model for the analysis of windturbine," Journal of Energy, p. 7:73-78, 1983.

[56] "MECH 5304 Lecture Notes," Carleton University, 2016.

[57] M. A. Hossain, Z. Huque and R. R. Kammalapati, "Propagation of Shock on NREL Phase VI Wind Turbine Airfoil under Compressible Flow," Journal of Renewable Energy, 2013.

[58] S. Moaveni, "Finite Element Analysis," in Finite Element Analysis, Upper Saddle River, New Jersey, Pearson Education, 2003, p. pg 652.

[59] Symscape , "NACA 4415 Airfoil," 2311 2007. [Online]. Available: http://www.symscape.com/node/562. [Accessed 1312017 ].

[60] B. Johnson, "Computational Fluid Dynamics (CFD) Modelling of Renewable Energy Turbine Wake Interactions," Ph.D. Dissertation, University of Central Lancashire, Preston, England, 2015.

[61] P. Moin and K. Mahesh, "DIRECT NUMERICAL SIMULATION: A Tool in Turbulence Research," Annu. Rev. Fluid Mech., vol. 30, p. 539-78, 1998.

[62] S. Deck, "Zonal-Detached-Eddy Simulation of the Flow Around a High-Lift Configuration," AIAA, vol. 43, no. 11, pp. 2372-2374, 2005.

[63] ANSYS® Academic Research, Release 15.0, Theory Guide, 2.1.1. Statistical Turbulence Models and the Closure Problem, ANSYS, Inc..

[64] F. R. Menter, "Improved Two-Equation k- $\omega$ Turbulence Models for Aerodynamic Flows," NASA AMES Research Center, Moffett Field, California, 1992.

[65] B. Launder, "The Numerical Computation of Turbulent Flow Computer Methods," Computer Methods in Applied Mechanics and Engineering, vol. 3, pp. 269-289, 1974.

[66] G. Kalitzin, G. Medic, G. Iaccarino and P. Durbin, "Near-wall behavior of RANS turbulence models and implications for wall functions," Journal of Computational Physics, pp. 265-291, 2005.

[67] ANSYS ${ }^{\circ}$, Academic Research, Release 11.0, ANSYS CFX-Solver Theory Guide, Turbulence and Wall Function Theory, ANSYS, Inc...

[68] ANSYS ${ }^{\circledR}$ Academic Research, Release 16.2, Help System, 15.4.1. Steady-state Time Scale Control, ANSYS, Inc.

[69] ANSYS Inc., "1.2.1. Steady State and Transient Flows," ANSYS ${ }^{\circledR}$, Academic Research, Release 15.0, ANSYS CFX-Solver Theory Guide, 2008.

[70] ANSYS® Academic Research, Release 16.2, Help System, 1.2.1. Steady State and Transient Flow, ANSYS, Inc. .

[71] ANSYS Inc., " Transient Flow Modeling," ANSYS®, Academic Research, Release 13.0,ANSYS CFX-Solver Theory Guide.

[72] "ANSYS® Academic Research, ANSYS 2009, Introduction to CFX, Transient Simulations, ANSYS, Inc.". 
[73] C.-W. Shu, "A brief survey on discontinuous Galerkin methods in computational fluid dynamics*," 力学进展, vol. 43, pp. 541-553, 2013.

[74] ANSYS ${ }^{\circledR}$ Academic Research, Release 15.0, ASYS CFX-Solver Theory Guide, 11: Discretization and Solution Theory, ANSYS, Inc. , 2013. [Online].

[75] ANSYS® Academic Research, Release 16.2, Help System, ANSYS, Inc., "11.2.2. Linear Equation Solution".

[76] ANSYS® Academic Research, Release 16.2, Help System, 15.10.1 Residuals, ANSYS, Inc..

[77] "ANSYS® Academic Research, ANSYS 2009, Introduction to CFX, Solver Settings , ANSYS, Inc.".

[78] N. O. Jensen, "A note on wind generator interaction," DTU Orbit, Roskilde, 1983.

[79] Statoil , "Our offshore wind projects," 2017. [Online]. Available: https://www.statoil.com/en/what-we-do/new-energy-solutions/our-offshore-windprojects.html?gclid=CjwKEAjwlKLHBRDztKr6wMnRthMSJAALcTsFrfhKiak_btlK5XnJWno0Yikc2onQhVFYpQj9jQSZhoCFYTw_wcB.

[80] M. B. Christiansen and C. B. Hasager, "Wake effects of large offshore wind farms identified from satellite SAR," Remote Sensing of Environment, vol. 98, no. 2-3, p. 251268, 2005.

[81] Vattenfall, "Horns Rev Offshore Wind Farm," 2017. [Online]. Available: http://powerplants.vattenfall.com/horns-rev. [Accessed 8 April 2017].

[82] E. Duque and S. H. C. van Dam, "Navier-Stokes simulations of the NREL combined experiment phase II rotor," in AIAA Aerospace Sciences Meeting and Exhibit, Reno, NV, 1999.

[83] L. Myers and A. Bahaj, "Experimental analysis of the flow field around horizontal axis tidal turbunes by use of scale mesh disk rotor simulations," Ocean Engineering, p. 218227, 2010.

[84] P. Réthoré, N. Troldborg, F. Zahle and N. Sørensen, "Comparison of the Near Wake of Different Kinds of Wind Turbine CFD Models," Wake Conference. Gotland, p. 7, 2011.

[85] R. Mikkelsen, J. Sørensen, S. Øye and N. Troldborg, "Analysis of Power Enhancement for a Row of Wind Turbines Using the Actuator Line Technique," Journal of Physics: Conference Series 75, 2007.

[86] F. Zahle, N. Sørensen and J. Johansen, "Wind turbine rotor-tower interaction using an incompressible overset grid method," Wind Energy, vol. 12, no. 6, p. 594-619., 2009.

[87] W. Miller, J. Sitaraman and D. Mavriplis, "Large Scale Simulations of Wind Farm Aerodynamics in Turbulent Atmospheric Inflow Conditions," University of Wyoming, 2013.

[88] Lawrence Livermore National Laboratory, "Predicting Wind Power with Greater Accuracy," May 2014. [Online]. Available: https://str.llnl.gov/april-2014/miller. [Accessed 8 April 2017].

[89] A. Namiranian, "3D Simulation of a 5MW Wind Turbine," Master's Thesis, Dept. of Mechanical Engineering, Blekinge Institute of Technology, Karlskrona, Sweden, 2011. 
[90] S. McTavish, "Identification of Wind Turbine Testing Practices and Investigation of the Performance Benefits of Closely-Spaced Lateral Wind Farm Configurations", Ph.D. dissertation,Dept. of Mech. and Aero. Eng., Carleton University," Ottawa, ON, 2013.

[91] Google Maps, " Main Duck Island," Google Inc., 2016. [Online]. Available: https://www.google.ca/maps/place/Main+Duck+Island/@43.9284017,77.1782372,141446m/data=!3m1!1e3!4m5!3m4!1s0x89d7f2c07d838893:0xea1567324a8 e5c5!8m2!3d43.9297471!4d-76.6268306!6m1!1e1. [Accessed 2005 2016].

[92] M. Samorani, "The Wind Farm Layout Optimization Problem," Alberta School of Business, University of Alberta, Edmonton, AB, 2013.

[93] R. Townsend, "Main Duck Island History," Lake Ontario Offshore Racing, [Online]. Available: http://loor.ca/lake-ontario-300/history/main-duck-island-history/.

[94] Helimax Energy Inc., "Analysis of Future Offshore Wind Farm Development in Ontario," Prepared for Ontario Power Authority, Toronto, ON, 2008.

[95] "Innovative Design Approaches for Large Wind Turbine Blades," Warren, RI, Sandia Report, WindPACT Blade System Design Studies, 2003.

[96] Sandia National Laboratories, "Innovative Design Approaches for Large Wind Turbine Blades," Albuquerque, New Mexico, 2003.

[97] F. Menter, "A comparison of some recent eddy-viscosity turbulence models," Fluids Eng., 1996, pp. 514-519.

[98] ANSYS® Academic Research, ANSYS 2009, Introduction to CFX, Interfaces,Sources and Additional Variables, ANSYS, Inc..

[99] ANSYS® Academic Research, ANSYS 2009, Introduction to CFX, Moving Zones, ANSYS, Inc.

[100] ANSYS ${ }^{\circledR}$ Academic Research, Release 16.2, Help System, Domain Interface Modelling , ANSYS, Inc..

[101] ANSYS®, Academic Research, Release 15.0, ANSYS CFX-Solver Theory Guide, 8.6.6.1.4. Frame Change Option $=$ Transient Rotor Stator, ANSYS, Inc., 2013.

[102] S. Wallin, "Engineering turbulence modelling for CFD with a focus on explicit algebraic Reynolds stress models," Technical Reports from Royal Institute of Technology, Department of Mechanics, Stockholm,Sweden, 2000.

[103] ANSYS Inc., "11.1.1.4. Advection Term," ANSYS ${ }^{\circledR}$, Academic Research, Release 16.2, ANSYS CFX-Solver Theory Guide.

[104] ANSYS Inc., 17.3.4. Convergence Control Parameters, Academic Research, Release 16.2, Help System.

[105] ANSYS® Academic Research, Release 15.0, ASYS CFX-Solver Theory Guide, 1: Courant Number, ANSYS, Inc., 2013.

[106] H. Trac and U.-L. Pen, "A Primer on Eulerian Computational Fluid Dynamics for Astrophysics," Publications of the Astronomical Society of the Pacific,, p. 115:303-321, 2003.

[107] ANSYS ${ }^{\circledR}$ Academic Research, Release v1.3, ASYS FLUENT Training, Solver Settings, ANSYS, Inc., 2006. 
[108] P. Moin, Fundamentals of Engineering Numerical Analysis, New York, NY: Cambridge University Press, 2010.

[109] ANSYS, Inc., "15.4.2.8.2.1. Timestep Initialization," ANSYS ${ }^{\circledR}$ Academic Research, Release 16.2, ANSYS CFX-Solver Theory Guide.

[110] L. M. Tossas and S. Leonardi, "Wind Turbine Modeling for Computational Fluid Dynamics," NREL, Mayaguez, Puerto Rico , 2010-2012.

[111] Siemens, "Offshore Direct Drive Wind Turbine SWT-6.0-154," 2017. [Online]. Available: http://www.siemens.com/global/en/home/markets/wind/turbines/swt-6-0-154.html. [Accessed 0203 2017].

[112] Qpedia, "Understanding Hot-Wire Anemometry," Thermal Minutes, 2007.

[113] E. Hau., Wind Turbines: Fundamentals, Technologies, Application and Economics., Springer, 2005.

[114] L. Chamorro and F. Sotiropoulos, "Turbulent flow inside and above a wind farm: A windtunnel study.," Energies 2011,4, 1916-1936.

[115] D. Elliott, "Status of wake and array loss research," in Windpower Conference Palm Springs, 1991.

[116] S. Jayanti, The Turbulent Closure Problem, NPTEL Online Certification Course, 2016.

[117] H. Snel, "Review of Aerodynamics for Wind Turbines," Wind Energy, pp. 6:203-211, 2003.

[118] The Office of Energy Efficiency \& Renewable Energy, "THE INSIDE OF A WIND TURBINE," U.S. Department of Energy, [Online]. Available: https://energy.gov/eere/wind/inside-wind-turbine-0. [Accessed 193 2017].

[119] R. Barthelmie, S. Frandsen, N. Nielsen, S. Pryor, P. Réthoré and H. Jørgensen, "Modelling and measurements of power losses and turbulence intensity in wind turbine wakes at middelgrunden offshore wind farm," Wind Energy, p. 10:217-228, 2007.

[120] J. Sørensen, W. Shen and X. Munduate, "Analysis of wake states by a full-field actuator disc model," Wind Energy, p. 1:73-88, 1998.

[121] S. Ivanell, J. Sørensen, R. Mikkelsen and D. Henningson, "Analysis of numerically generated wake structures," Wind Energy, p. 12:63-80, 2009.

[122] "fotosearch,"

[Online].

Available: http://sr.photos2.fotosearch.com/bthumb/CSP/CSP060/k18545590.jpg. [Accessed 15 March 2017].

[123] J. Sørensen and W.Shen, "Numerical modeling of wind turbine wakes," Journal of Fluids Engineering, p. 124:393-399, 2002.

[124] Vestas, "V90 3.0 MW," 2013.

[125] National Oceanic and Atmospheric Administration, "Office of the Coast Survey".

[126] Google Maps , "Main Duck Island to Galloo Islands," 2017. [Online]. Available: https://www.google.ca/maps/dir/main+duck+island/galloo+islands/@43.9175965,76.6616636,35089m/data=!3m1!1e3!4m13!4m12!1m5!1m1!1s0x89d7f2c07d838893:0xe a1567324a8e $5 \mathrm{c} 5 ! 2 \mathrm{~m} 2 ! 1 \mathrm{~d}-$ $76.6268306 ! 2 \mathrm{~d} 43.9297471$ ! $1 \mathrm{~m} 5$ !1 m1!1s0x89d81 f98c9b14f91:0x36bc3d68c5365d1e!2m 2 !1d-76.4. 
